\title{
Succesfully managing alliance portfolios: An alliance capability view
}

Citation for published version (APA):

Saebi, T. (2011). Succesfully managing alliance portfolios: An alliance capability view. [Doctoral Thesis, Maastricht University]. Datawyse / Universitaire Pers Maastricht. https://doi.org/10.26481/dis.20110622ts

Document status and date:

Published: 01/01/2011

DOI:

$10.26481 /$ dis.20110622ts

Document Version:

Publisher's PDF, also known as Version of record

\section{Please check the document version of this publication:}

- A submitted manuscript is the version of the article upon submission and before peer-review. There can be important differences between the submitted version and the official published version of record.

People interested in the research are advised to contact the author for the final version of the publication, or visit the DOI to the publisher's website.

- The final author version and the galley proof are versions of the publication after peer review.

- The final published version features the final layout of the paper including the volume, issue and page numbers.

Link to publication

\footnotetext{
General rights rights.

- You may freely distribute the URL identifying the publication in the public portal. please follow below link for the End User Agreement:

www.umlib.nl/taverne-license

Take down policy

If you believe that this document breaches copyright please contact us at:

repository@maastrichtuniversity.nl

providing details and we will investigate your claim.
}

Copyright and moral rights for the publications made accessible in the public portal are retained by the authors and/or other copyright owners and it is a condition of accessing publications that users recognise and abide by the legal requirements associated with these

- Users may download and print one copy of any publication from the public portal for the purpose of private study or research.

- You may not further distribute the material or use it for any profit-making activity or commercial gain

If the publication is distributed under the terms of Article $25 \mathrm{fa}$ of the Dutch Copyright Act, indicated by the "Taverne" license above, 


\section{SUCCESSFULLY MANAGING ALLIANCE PORTFOLIOS:}

AN ALLIANCE CAPABILITY VIEW 
(C) Tina Saebi, Maastricht 2011

ISBN: 9789461590619

Production: Datawyse I Universitaire Pers Maastricht Cover design: Flickr.com 


\title{
SUCCESSFULLY MANAGING ALLIANCE PORTFOLIOS: AN ALLIANCE CAPABILITY VIEW
}

\author{
PROEFSCHRIFT \\ ter verkrijging van de graad van doctor aan de Universiteit Maastricht, \\ op gezag van de Rector Magnificus, Prof. mr. G.P.M.F. Mols, \\ volgens het besluit van het College van Decanen \\ in het openbaar te verdedigen op \\ woensdag 22 juni 2011 om 12.00 uur
}

door

Tina Saebi

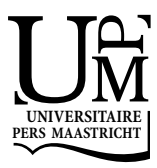




\section{Promotor:}

Prof. dr. Geert Duysters

\section{Co-Promotor :}

Prof. dr. Ard-Pieter de Man, Vrije Universiteit Amsterdam

\section{Beoordelingscommissie:}

Prof. dr. John Hagedoorn, voorzitter

Prof. dr. Tom Elfring, Vrije Universiteit Amsterdam

Prof. dr. Friso den Hertog 


\section{ACKNOWLEDGEMENTS}

I once read 'The main product of a PhD study is the person that comes out if it in the end; the book is only secondary'.

Reflecting about the last years, this statement could not be truer. It has been a journey, where I had the opportunity to challenge myself, to go beyond my boundaries and to learn many skills not only for a career in academia but for life. Along this journey, I was blessed to be in the company of so many brilliant and good people, who each have contributed to the success of this project in their own way.

It has been a true blessing to be the doctoral student of Professor Geert Duysters and Professor Ard-Pieter de Man, who have always been constructive and encouraging in their supervision. Both have always provided me with a positive outlook and support, especially in times where I was mired in self-doubt.

I thank the members of my assessment committee, Professors John Hagedoorn, Tom Elfring and Friso den Hertog, for reviewing my dissertation. I would like to take this opportunity and especially thank Professor Hagedoorn for paving my way to a PhD. As supervisor for my master thesis he introduced me to the art of academic research and encouraged me to pursue this path. Moreover, I would like to thank Professor Luc Soete and Professor Robin Cowan for their encouragement and academic insight over these last years.

During my fieldtrip to Wuhan University of Technology, I had the invaluable opportunity to apply my research on alliances to the context of China. With the collaboration and never-ending hospitality of my Chinese colleagues, especially Professor Xie Kefan, Qinqin Dong and Du Jingshu, I had the chance to experience a successful academic Sino-Western alliance first hand.

I would like to thank my dear PhD classmates, Asel Doranova, Sergey Filippov, Donatus Ayitey, Radhika Perrot and Evans Mupela for a great time. It is beautiful to see how friendships bloom under the hard regime of maths 
and economic growth classes. During my PhD time at UNU-MERIT I have met so many amazing people who have shared their friendship with me. A big thank you goes to Piret Kukk, Sabine Fuss, Massimiliano Volpi, Shuan SadreGhazi, Ying Zhang, Yong Qian, Iman Rabajzadeh, Daniel Vertesy and Flavia Carvalho -to only name a few. I also would like to thank Dr. Ionara Costa for her insight and feedback in the beginning stages of this dissertation, and Nico Rasters, friend and data management specialist, who has helped me with my online survey.

I am indebted to Christian Kerckhoffs, who shared his statistical expertise and friendship even before I started the PhD program.

Special thanks go to Wilma Coenegracht for her kindness and invaluable advice over these years. I would like to thank Eveline in de Braek for her continuous support in $\mathrm{PhD}$ related administrative matters and creating a welcoming environment for the PhD students. I also extend my gratitude to Silvana de Sanctis, Monique Readts, Eric Engelen, Ad Notten, Mourik-Jan Heupink and Herman Pijpers for their administrative and technical support.

I would like to thank my parents, Fataneh and Mehrdad Saebi, and my family for their unconfined support and trust in my capabilities. Especially, I am grateful to my uncle, Professor M. Laridjani, who has always encouraged me to find my own way and to appreciate the beauty of life. It is their love and strength that has kept me on my PhD path.

Finally, I would like to thank Ruediger, 'mein Fels in der Brandung', for reminding me of the importance of maintaining serenity and patience in face of life's struggles and for encouraging me to follow my dreams in life.

In the words of David Richo

"For all that has been: Thanks!

For all that will be: Yes!"

Tina Saebi

Maastricht, April 2011 


\section{CONTENTS}

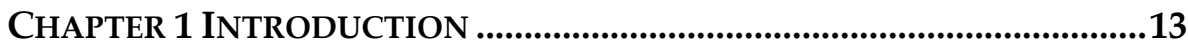

$1.1 \quad$ The strategic importance of alliances …………………………................13

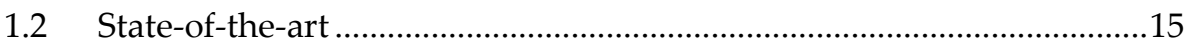

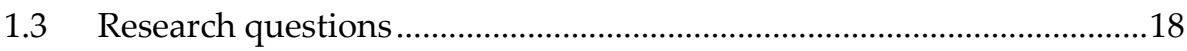

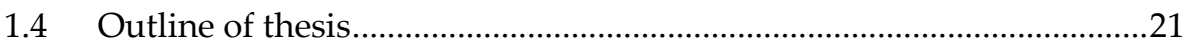

\section{Chapter 2 Theoretical Perspectives on Alliance (Portfolio)} FORMATION AND SUCCESS FACTORS ....................................23

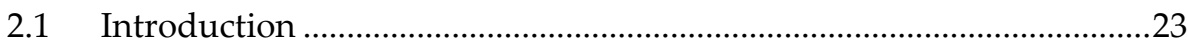

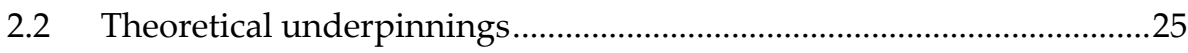

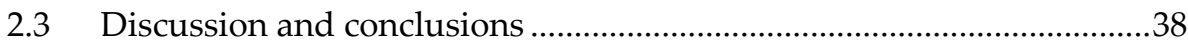

Chapter 3 Alliance CAPABility: AN Elusive Phenomenon...........43

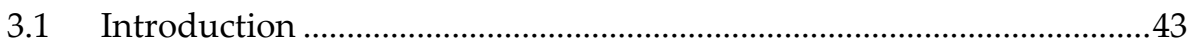

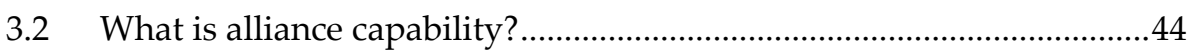

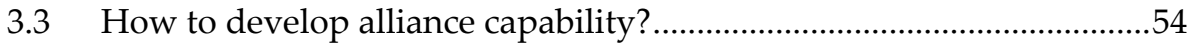

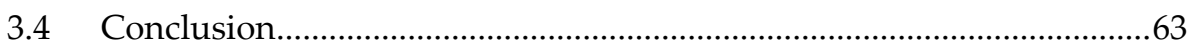

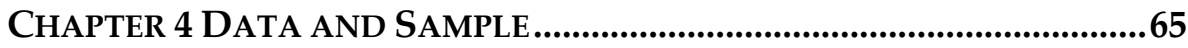

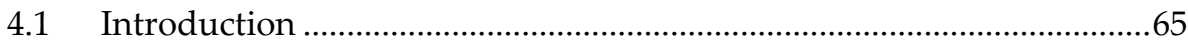

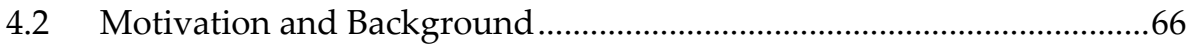

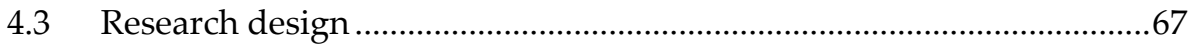

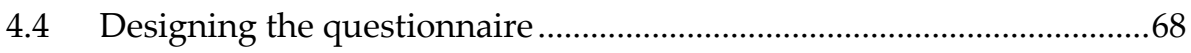

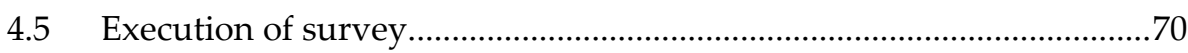

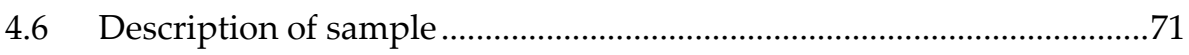

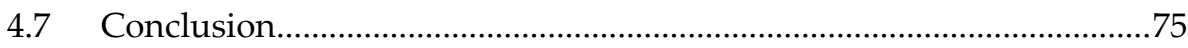




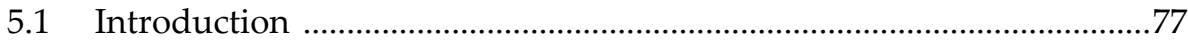

5.2 The roles of alliance experience and alliance mechanisms in raising alliance performance ............................................................................

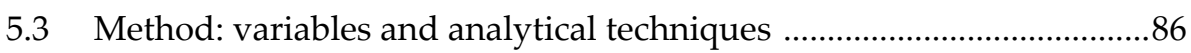

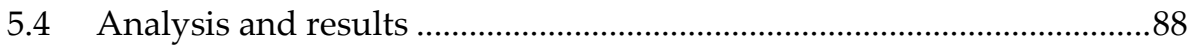

5.5 Discussion and conclusion ...................................................................

Chapter 6 DeVeloping Alliance Portfolio CAPAbility.................97

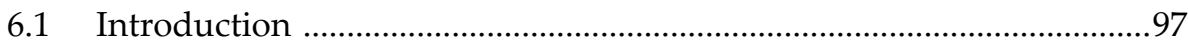

6.2 Conceptualising alliance capability on individual vs. portfolio level ..99

6.3 Developing alliance portfolio capability: the role of alliance

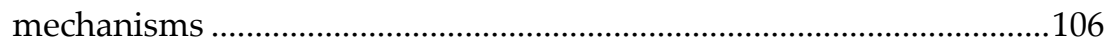

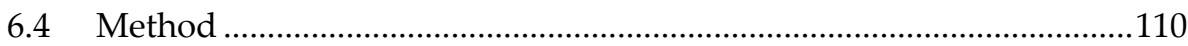

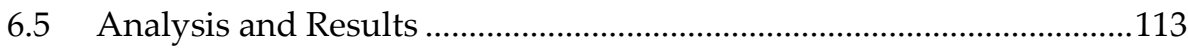

6.6 Discussion and Conclusion ............................................................117

Chapter 7 Managing Alliance Portfolio Diversity ................121

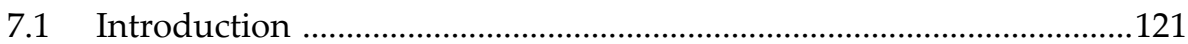

7.2 Understanding alliance portfolio diversity .............................................123

7.3 Managing alliance portfolio diversity ..................................................129

7.4 Method: variables and analytical techniques .....................................132

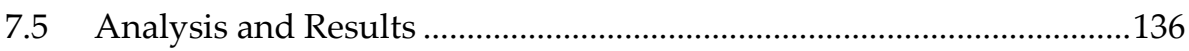

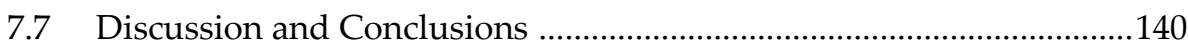

\section{CHAPTER 8 CONCLUSIONS, IMPLICATIONS AND FURTHER RESEARCH}

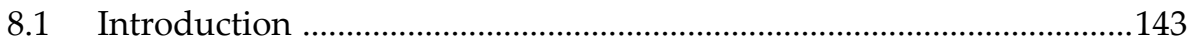

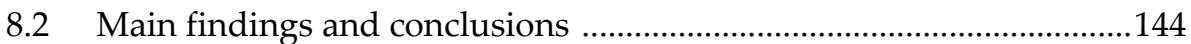

8.3 Contributions and their theoretical and managerial implications ......149

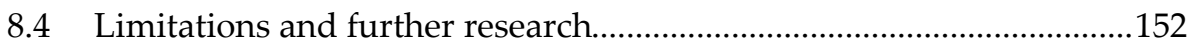

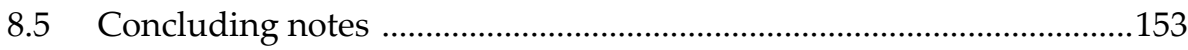


REFERENCES

APPENDIX

169

SAMENVATTING 173

CURRICULUM VITAE...............................................................................177 



\section{LIST OF TABLES}

Table 3.1: Alliance capability and related concepts in chronological order..... .46

Table 3.2: Alliance management mechanisms. .60

Table 3.3: Alliance mechanisms categorised in terms of main purpose in alliance capability building

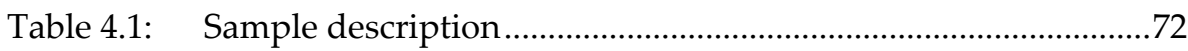

Table 4.2: $\quad$ Types of alliances used by companies ......................................73

Table 4.3: Alliance performance distribution ...............................................73

Table 4.4: Frequency of implemented alliance mechanisms .......................74

Table 5.1: Distribution of alliance experience................................................87

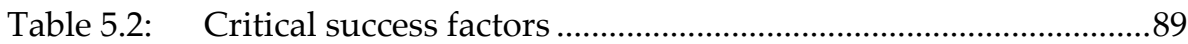

Table 5.3: Average adoption by absolute number of alliance mechanisms per alliance experience level (ANOVA) ................90

Table 5.4: Successful mechanisms given level of alliance experience........91

Table 6.1: What constitutes alliance portfolio capability? ..........................105

Table 6.2: $\quad$ Distribution of alliance portfolio size in sample .......................111

Table 6.3: Average adoption by absolute number of alliance mechanisms per alliance portfolio size category (ANOVA)

Table 6.4: Average adoption of alliance mechanisms types per alliance portfolio size category (ANOVA)

Table 6.5: Critical success factors for alliance portfolio performance ..... 116

Table 7.1: Distribution of functional activities in alliance portfolios .......134

Table 7.2: Distribution of partner diversity in alliance portfolios .............134

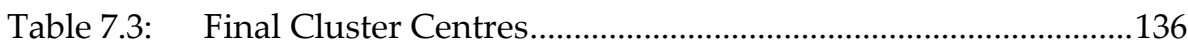

Table 7.4: $\quad$ Number of Cases in each Cluster ...............................................137

Table 7.5: Average adoption of absolute number of alliance mechanisms (ANOVA) .............................................................137

Table 7.6: Average adoption of categorised alliance mechanisms per alliance portfolio category (ANOVA) .......................................138

Table 7.7 Critical success factors for different alliance portfolio

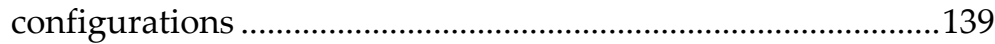

Table 8.1: $\quad$ Overview of effective alliance mechanisms .............................148 


\section{LIST OF FIGURES}

Figure 1.1: Outline of thesis........................................................................22

Figure 2.1: Alliance capability research versus traditional research............37

Figure 3.1: Life cycle of alliance relationships.............................................49

Figure 5.1: Relationship between the number of alliances and the success of the organisation's alliances........................................8 80

Figure 5.2: Levels of alliance capability development. ..................................84

Figure 7.1: Typology of alliance portfolio configurations ..........................124 


\section{CHAPTER1 INTRODUCTION}

"If you think you can go it alone in today's global economy, you are highly mistaken" Jack Welch, CEO of General Electrics

\subsection{The strategic importance of alliances}

Our study aims to investigate how firms can successfully manage a portfolio of alliances. This is an important issue as firms rarely actualise the full value creating potential of their alliances. Strategic alliances (hereafter also referred to as 'alliances') are commonly defined to involve at least two partner firms that aim to improve their competitive position and performance by the joint contribution of resources and knowledge and the sharing of benefits, risks and control of joint activities (Ireland et al., 2003; Yoshino and Rangan, 1995). Contrary to integrative agreements such as mergers and acquisitions, partner firms in alliances maintain their own corporate identities after the alliance is formed. An alliance network comprises direct alliance relationships (between the focal firm and its alliance partners) and indirect ties (partners-of-partners) (Knoke 2001:128). An alliance portfolio, however, is defined as the collection of simultaneously operating direct alliance relationships of the focal firm, also referred to as an egocentric alliance network (Baum et al., 2000; Rowley et al., 2000).

In the following, we will emphasise the importance of advancing research on alliance portfolio management and discuss research gaps in extant alliance literature. We then outline how we intend to address these research gaps in the course of this study.

The surge in alliance portfolio formation (Lavie, 2007) and the rising percentage of revenues being generated by alliance portfolios (Duysters and De Man, 2007) indicate the remarkable rise in the importance of these collaborative ventures for the competitive survival of many firms (Narula and Duysters, 2004; Margulis and Pekar, 2001). 
To respond to the increasing globalization of markets, shortening of innovation cycles and the search for new capabilities, firms no longer rely on a single high profile alliance but increasingly depend on various external partners to enhance their resource endowment (Eisenhardt and Schoonhoven, 1996), master strategic uncertainty (Hoffmann, 2007), access foreign markets (Parkhe, 1991), improve competitive positioning vis-à-vis rivals (Gimeno, 2004; Silverman and Baum, 2002) and share technology and knowledge (Dyer and Nobeoka, 2000; Kale, Singh, Perlmutter, 2000; Hagedoorn, 1993). Therefore, building a portfolio of alliances enables the firm to maximise its presence in not only its primary markets but in different (foreign) business sectors as well as to exploit and secure the differing competitive advantages of each location. In addition, a large alliance portfolio with a variety of alliance partners allows firms timely access to a diversity of information and resources. As a result, many firms are embedded in a dense network of alliance relationships with competitors, suppliers and customers often from a variety of industrial and national backgrounds (Hoffmann, 2007).

Despite the increasing strategic importance of alliance portfolios, firms seem to encounter severe problems in managing their alliances, as the success rate of alliances is fairly low. Average alliance success rates of 50\% (Duysters and De Man, 2007) testify to the difficulty of consistently attaining successful alliances. Alliance success has commonly been measured by both objective measures (e.g. profitability, market share, new product development, or sales growth) and subjective assessments of goal achievement and overall satisfaction (McCutchen, Swamidass and Teng, 2008). Notwithstanding the different measures of alliance performance, alliance success remains a rare phenomenon for many companies.

While firms aim for collaborative synergy, the nature of strategic alliances leaves the partners vulnerable to the many relational and structural obstacles that challenge the successful conduct of alliances. Especially the mutual interdependence and frequent simultaneous cooperation and competition between partners (Parkhe, 1993) are suggested to contribute to the premature termination of the alliance relationship, reflecting a business failure or an irresolvable conflict among partners (Kogut, 1989) that is likely to result in incurable losses of proprietary information and technology (Park and Ungson, 2001). 
Notwithstanding the many potential challenges, the current growth rate in alliance portfolio formation is suggested to continue (Lavie and Miller, 2008). It seems that while many firms are well aware of the strategic importance of alliances, only a few have mastered the ability to attain successful alliance portfolios. Therefore, the question how to attain successful alliance portfolios is appealing to both academics and practitioners.

\subsection{State-of-the-art}

By and large, two distinct research streams can be distinguished that address the question of how to create successful alliance portfolios. The first research stream generally adopts a social network perspective and emphasises the structural characteristics of firms' alliance portfolios (such as portfolio size and structure) and its implications for the firms' positioning in a network of alliances. The second research stream assumes a firm-level perspective and finds that a firm's organizational capability in managing alliances is the main determinant for alliance performance.

From a social network perspective (Burt, 1992; Coleman, 1990; Kogut, Shan and Walker, 1992), scholars suggest that a firm's network of alliances can itself present a source of strategic advantage as it facilitates access to unique resources and information (Gulati, 1999). Thus, how a firm positions itself in a network of alliances substantially influences its competitiveness (Dyer and Singh, 1998; Gulati, Nohria, and Zaheer, 2000). The firm's positioning in networks is defined by the configuration of its alliance portfolio (Hoffmann, 2007). Social network studies emphasise that alliance portfolio configuration is a complex concept consisting of multiple dimensions including the number, dispersion and redundancy of alliances and their linkage strength (Granovetter, 1985; Rowley, Behrens and Krackhardt, 2000). Therefore, as Hoffmann (2007:834) notes, the configuration of alliance portfolios determines

"(1) the quality, quantity, and diversity of information and resources to which the focal company has access, (2) the efficiency of the access to these network resources, and (3) the flexibility or stability of the focal company's position in the interorganizational field". 
As a result, a rising number of studies investigate the implications of structural characteristics of alliance portfolios for performance; such as portfolio size (Rothaermel and Deeds, 2006), partner diversity (Stuart, 2000), the number of direct and indirect ties (Ahuja, 2000), tie diversity (Powell, Koput and Smith-Doerr, 1996), and the strength of ties (Rowley et al., 2000). Consequently, how the focal firm configures its alliance portfolio is expected to influence its positioning in the larger context of inter-firm networks and eventually its competitiveness (Dyer and Singh, 1998; Gulati, 1998, 1999).

The second stream of alliance research adopts a firm-level perspective and finds that firms that score high alliance performance rates possess superior alliance management skills (Draulans et al. 2003; Kale, Dyer and Singh 2002; Anand and Khanna 2000; Sivadas and Dwyer 2000). This proficiency in alliance management has been termed alliance capability and is defined as the institutionalised approach to learning about alliance management in order to support the firm in the formation, operation and evaluation of its alliances. ${ }^{1}$ Firms with a high level of alliance capability have institutionalised managerial practices that guide and support alliance management as opposed to a 'trial and error' approach to alliance management. For instance, a standardized alliance partner selection process can present a repeatable pattern of action that has proven to be fruitful in managing alliances. Hence, alliance performance differences among firms are suggested to be due to heterogeneity in firms' level of alliance capability.

To develop alliance capability, scholars emphasise the importance of acquiring alliance experience through engaging in numerous alliances (Anand and Khanna, 2000; Rothaermel and Deeds, 2006) and using organizational mechanisms to translate alliance experience into accessible lessons on alliance management (Kale et al., 2002). Herewith, the 'alliance capability view' shifts away from the outcome implications of structural characteristics of alliance portfolios but directs the focus towards the learning processes and managerial practices that improve the firm's overall ability to manage alliances and hence raises performance rates.

\footnotetext{
${ }^{1}$ A detailed discussion of the definitions and construct of alliance capability will be provided in Chapter 3.
} 
A predominant share of this stream's contributions to the scholarly debate on alliance capability has centred on the management of individual alliances and only sparsely tackled the question what alliance capability needs to be developed for the effective management of firm's alliance portfolios. Only recently, a number of studies have proposed the importance of an 'alliance portfolio management capability' that addresses the particular managerial challenges that arise in a portfolio of alliances (Hoffmann, 2005, 2007; Sarkar, Aulakh and Madhok, 2009). These studies advise to shift away from treating each alliance as a standalone transaction but to take the entire alliance portfolio as the unit of analysis. As alliances do not create value independent of each other (Gulati, 1998), an alliance portfolio can create more value through synergy than the sum total of each alliance (Bamford and Ernst, 2002). Hence, Sarkar et al. (2009) emphasise that "managing the portfolio as a coordinated, holistic collective is thus likely to create value beyond what can be accomplished if each were managed separately" (p.588). In line with this logic, Hoffmann (2007) states that "what really matters is not the success or failure of a single alliance but that the company will reach its strategic goals with the bundle of its alliances" (p.828).

Thus, scholars indicate that alliance capability needs to be differentiated in terms of single vs. portfolio alliance management (Hoffmann, 2007; Sarkar et al., 2009; Schreiner, Kale and Corsten, 2009). For instance, while alliance capability at the single alliance management level needs to address challenges that arise at different stages of the life cycle of an individual alliance relationship (Schreiner et al., 2009), alliance management at the portfolio level attends the development of a portfolio strategy and tackles the monitoring and coordination of the alliance portfolio (Hoffmann, 2005). Along the same lines, Sarkar et al (2009, p.584) note that alliance portfolio capability needs to be directed at alliance portfolio formation, development and integration. A number of managerial tools have been identified to facilitate such an approach, such as partner programs and alliance databases (Duysters, De Man and Wildeman, 1999; Heimeriks et al., 2008). 


\subsection{Research questions}

Reflecting upon both streams of alliance research, we find that neither body of literature provides a comprehensive understanding of how to manage alliance portfolios successfully. Alliance literature drawing on social network theory emphasises the importance of alliance portfolio characteristics for value creation but allows for no further insight on how to manage alliance portfolios successfully. At large, alliance capability literature has mainly addressed managerial tasks at the single alliance management level. The few studies that particularly address the need for alliance portfolio capability are highly valuable but generate only fragmented insight into effective alliance portfolio management. Hence, we suspect that the inability of firms to attain successful alliance portfolios might be due to a lack of research on alliance portfolio management. Therefore, adopting an alliance capability view,$^{2}$ we dedicate this dissertation to the following overall question:

How can firms develop alliance capability that effectively supports the management of their alliance portfolios?

In answering this question, we address a number of critical research gaps in current alliance literature. Firstly, we need to clarify the definition of alliance capability and its organizational determinants. Conceptually speaking, we find that literature on the construct of alliance capability is plagued with inconsistencies and ambiguities, as it is reflected by the numerous terms used and various definitions provided. In particular, alliance literature seems to disagree about the constituents of alliance capability. Therefore, we find it crucial to address the question:

\section{(1) What is alliance capability?}

Secondly, the conceptual uncertainty has severe consequences for the empirical methods used in extant alliance literature. On the empirical level, a number of studies strive to assess how firms can develop alliance capability and thus raise alliance performance. Within this field, we note that empirical

\footnotetext{
${ }^{2}$ The reason why we adopt an alliance capability view will be discussed in Chapter 2
} 
methods to proxy and measure the organizational determinants of alliance capability and assess their relationship to alliance performance tend to be frail. While the theoretical discourse on capability building is advanced, empirical applications in extant literature do not seem to capture sufficiently the complexity of the alliance capability development process. In particular, the relationship between alliance experience, alliance mechanisms, and alliance performs remains ambiguous.

(2) How can firms build alliance capability in order to raise the performance rate of their alliances?

Thirdly, prior research has given us reasonable grounds to expect that alliance capability - with its underlying managerial practices - can be differentiated into $a$ ) single alliance management capability that is geared towards the management of individual alliance relationships (Schreiner et al. 2009) and (b) alliance portfolio management capability (Hoffmann, 2005; Sarkar et al., 2009). However, conceptual research on this issue remains fragmented. Therefore, to explore how alliance capability on the single alliance level is different from alliance capability on the portfolio level, we need to differentiate clearly:

(3) How does the management of alliance portfolios reach beyond the tasks of managing individual alliances?

Fourthly, this differentiation has largely remained conceptual in nature. To substantiate such an argument, a number of studies have empirically tested the different management practices needed to manage small vs. large alliance portfolios (e.g. Heimeriks et al., 2008; Hoffmann, 2005; Duysters et al., 1999). That is, expanding alliance portfolio size (i.e. increasing the number of simultaneously operating alliance relationships) is associated with different managerial challenges and hence the need for more 'advanced' alliance management practices (Draulans et al., 2003). However, with the single exception of a study by Heimeriks et al. (2008), we notice a critical lack of large-scale empirical research to differentiate the alliance mechanisms associated with the successful management of alliance portfolios of different sizes. Therefore, to advance empirical research on the effective management of alliance portfolios, we intend to research: 
(4) With an increase in alliance portfolio size, which alliance management practices are particularly effective to raise the performance of the alliance portfolio?

Finally, in order to complete our analysis on the effective management of alliance portfolios, we need to examine the phenomenon of alliance portfolios in its entirety. In addition to portfolio size, alliance portfolio diversity is another important feature characterising the configuration of alliance portfolios and thus influencing firm performance (Hoffmann, 2007). Alliance portfolio diversity is defined as the variety in sources of information, knowledge and resources that a firm has access to by virtue of its alliance portfolio (Harrison and Klein, 2007). For instance, by engaging with different types of alliance partners (e.g. partners from different industries) the firm gains access to a variety of different information and resources, which can lead to a competitive advantage in its strategic field (Koka and Prescott, 2002; McEvily and Zaheer, 1999). So far, largely drawing on social network theory, alliance studies have investigated the merits and drawbacks of alliance portfolio diversity for firm innovativeness and performance (Beckman and Haunschild, 2002; Bae and Gargiulo, 2004; Baum, Calabrese and Silver, 2000; George, Zahra, Wheatley and Khan, 2001; Rowley, Behrens and Krackhardt, 2000, Stuart, 2000). However, while this stream assesses the implications of alliance portfolio diversity, little insight is offered on how firms can address and manage challenges that arise with diversity in alliance portfolios. More specifically, the issue of managing alliance portfolio diversity has not been examined sufficiently from an alliance capability view. Therefore, to complete our analysis on successful alliance portfolio management, we intend to research:

(5) What managerial challenges arise with alliance portfolio diversity and which alliance mechanisms are most effective at addressing these challenges?

By addressing these questions, we aim to generate a more comprehensive insight into how firms can develop alliance capability to successfully manage alliance portfolios. 


\subsection{Outline of thesis}

As the title of this dissertation suggests, we adopt an alliance capability view in order to research the effective management of alliance portfolios. Chapter 2 provides an extensive discussion of various theoretical views on alliance portfolio formation and success factors. Discussing these theories, we provide the reader with a comprehensive overview of different theories and derive a set of arguments on why we find the alliance capability view particularly suited to guide our research on alliance portfolio management.

Chapter 3 derives a definition of alliance capability by discussing its constituent elements. By critically discussing state-of-the-art literature, we address important inconsistencies and ambiguities regarding the notion of alliance capability in extant alliance literature.

Chapter 4 describes our research strategy and design and presents a brief overview of our data sample.

Chapter 5 examines empirically how firms can build alliance capability. In particular, we test more fine-grained empirical measures to capture the organizational determinants of alliance capability and their relationship to alliance performance.

Chapter 6 focuses on the development of alliance capability for alliance portfolio management. To this end, we examine the specific managerial challenges that arise at the portfolio level of alliance management. We investigate empirically which alliance mechanisms are most effective in supporting the management of large alliance portfolios.

Chapter 7 examines the various managerial challenges that arise with different types of alliance portfolio diversity and provides empirical proof for which alliance mechanisms are most effective in raising alliance portfolio performance.

Chapter 8 synthesises the results of our theoretical and empirical chapters and draws conclusions with regard to our research questions. 
Figure 1.1: $\quad$ Outline of thesis

Chapter 2 Theoretical perspectives on alliance portfolio formation and success factors

Chapter 3 Alliance capability: an elusive phenomenon

Chapter 4 Data and Sample

Chapter 5 Building alliance capability

Chapter 6 Developing alliance portfolio capability

Chapter 7 Managing alliance portfolio diveristy

Chapter $8 \quad$ Conclusions 


\section{Chapter 2 Theoretical Perspectives on Alliance (PorTfolio) Formation AND SucCess}

\section{FACTORS}

\subsection{Introduction}

The surge in alliance portfolio formation accompanied by the increasing share of revenue generated by alliance portfolios signifies the growing strategic importance of alliances for the competitive survival of many firms. A recent study on the evolution of alliance portfolios in the U.S software industry 1990-2001 demonstrates how the average number of alliances per company increased from four to more than 30 alliances during this decade (Lavie, 2007). As a result, we can observe that firms engage in multiple alliance relationships simultaneously, often with partners from different industrial and national backgrounds. However, research on alliance portfolios indicates a significant heterogeneity in performance rates among companies (Duysters and de Man, 2002, 2007). Average alliance failure rates range from $40 \%$ to $70 \%$ among companies (Bleeke and Ernst, 1993; Park and Ungson, 2001) illustrate that some companies are more able to attain successful alliance portfolios than other firms. Two important questions arise: (1) what drives the trend towards building large portfolios of alliances (2) how can companies attain successful alliance portfolios?

Regarding the first question, alliance research has extensively documented the manifold motives of companies to enter into strategic alliances; ranging from reducing transaction costs (Kogut, 1988), accessing valuable resources (Chung, Singh and Lee, 2000; Das and Teng, 2000; Eisenhardt and Schoonhoven, 1996; Hagedoorn, 1993; Lavie, 2006), to learning from partner firms (Inkpen, 2000; Dyer and Nobeoka, 2000; Kale, Singh, Perlmutter, 2000). Commonly adopted theories to study the rationale for alliance formation include organizational economics as well as resource-based and knowledgebased theories of the firm. 
We will refer to these three research streams as 'original alliance research' as their common unit of investigation is the individual alliance. ${ }^{3}$ Only recently, drawing upon social network theory, alliance studies suggest rationales of firms to build and maintain alliance portfolios that reach beyond the motivations to enter into individual alliances.

Regarding the second question, we observe that the quest for the ultimate key to alliance success is not a novel endeavour. Triggered by the growing strategic importance of alliances and the fact that firms are rarely able to tap into the full value creating potential of their alliances, we find a large share of alliance literature dedicated to unravel the factors that contribute to the success of alliances. However, what seems to be 'new' in the field of alliance research is a shifted focus in the unit of analysis. While original alliance research has focussed on the individual alliance, social network studies advise to shift away from treating each alliance as a standalone transaction but to take the entire alliance portfolio as the unit of analysis (Gulati, 1998). Accordingly, while original alliance research proposes the importance of structural and relational characteristics of the individual alliance relationship to determine success, social network scholars emphasise that "what really matters is not the success or failure of a single alliance but that the company will reach its strategic goals with the bundle of its alliances" (Hoffmann 2007 p.828). Hence, alliance research building upon social network theory stresses the implications of different alliance portfolio configurations (in terms of size and structure) for the overall success of the portfolio. A third unit of analysis is introduced by alliance capability scholars that direct the focus to the organizational level of the firm; proposing that a firm's ability to attain successful alliances is a function of its managerial capabilities.

The recent developments towards building large alliance portfolios call for a critical reflection about the meaningfulness of these theories to understand how firms can attain successful alliance portfolios. Especially with regard to 'original alliance research' we need to evaluate how well these theories are able to capture the importance of building alliance portfolios and how well

\footnotetext{
${ }^{3}$ Terming these three theories as 'original alliance research' does not intend to disregard their distinctiveness in theoretical views and logic. It is merely intended to highlight the differentiation between these three theories and other theories that we intend to introduce in the course of this chapter.
} 
they explain why some firms are more able to attain successful alliance portfolios than other firms. Therefore, we will present and discuss alliance research building upon alliance capability view and social network theory and make our case why these two theoretical stances provide complementary and comprehensive insights into the success factors of alliance portfolios.

In the remaining of this chapter, we will briefly present each theory and elaborate on how alliance research has drawn upon these theories to addresses the conditions of alliance (portfolio) formation, sources of instability and factors influencing alliance success. We conclude this chapter with a thorough discussion and evaluation of the explanatory power of these theories with regard to our two questions.

\subsection{Theoretical underpinnings}

In the following, we present the essential arguments of transaction cost theory, knowledge- and resource-based views of the firm, social network theory and dynamic capability view on alliance (portfolio) success factors. Alliance studies that have drawn upon either theory have generated comprehensive accounts of potential causes for relational conflict and alliance failure (e.g. Bleeke and Ernst, 1993; Douma et al., 2000; Hoffmann and Schlosser, 2001). All of these theories have been vastly applied in alliance literature to study the conditions for successful alliances (Hunt, Lambe and Wittmann, 2002; McCutchen, Swamidas and Teng, 2008). Alliance scholars building on transaction cost theory suggest that alliance success is significantly influenced by the appropriate choice of governance (Williamson, 1985; Hennart, 1988; Pisano, 1989; Osborn and Baughn, 1990), while proponents of the social exchange theory find relational factors such as trust (Ring and Van de Ven, 1992; Gulati, 1995) and commitment (Anderson and Weitz, 1992; Moorman et al., 1992) to drive alliance success. Resource-based alliance scholars emphasise the role of strategic fit in terms of complementary resources to determine alliance success (Sarkar et al., 2001; Harrison, Hitt, Hoskisson and Ireland, 1991; Hitt, Harrison and Ireland, 2001c; Ireland et al., 2001b). More recently, alliance research building on social network theory illustrate how the configuration of alliance portfolios (in terms of size and structure) significantly determine the value firms can generate from their portfolio of 
alliances, while dynamic capability scholars emphasise the important role of organizational managerial capabilities to attain successful alliance portfolios. To derive why certain factors are deemed important, we first briefly review the different rationales for alliance (portfolio) formation that each theory suggests. The reader will notice that a theory's view on alliance (portfolio) formation rationale is closely related to the theory's understanding of alliance (portfolio) success factors.

\subsubsection{Transaction cost theory: the importance of governance}

Transaction cost theory (Coase, 1937; Williamson 1985) has been widely adopted to study strategic alliances formation as it addresses the characteristics of economic activity that encourage managers to choose between vertical integration (e.g. mergers and acquisition), inter-firm cooperation (e.g. strategic alliances) and market exchange. Dating back to Coase (1937), firms are driven by the attempt to minimize the sum of transaction and production costs. Presuming opportunistic behaviour of economic agents (Hesterley et al 1990) and incomplete contracts (Hart, 1995), proponents of the transaction cost theory expect the potential consumer/supplier to default on the transaction agreement and thus lead to the failure of the market transaction (Klein, Crawford and Alchian 1978). In anticipation of opportunistic behaviour, business partners are inclined to write and enforce contracts to limit opportunistic actions - all of which generate transaction costs. As an alternative, when the transaction costs of market exchange are too high, firms can internalise the activity to control transaction costs efficiently. Nevertheless, such internalisation (e.g. mergers and acquisitions, internal development) can bear substantial production costs from coordinating activities in-house, such as the costs incurred from producing and managing the product internally. Hence, when the costs of market exchange (transaction costs, incomplete contracts) and internal development (costs of producing in-house) are too high, alliances are considered an appropriate alternative governance structure, as alliances combine features of internalisation with market exchange ${ }^{4}$. As opposed to internalisation, an alliance allows the partner firms to decrease the costs of production through joint coordination of the activity.

\footnotetext{
4 For the interested reader, Kogut (1988) provides an insightful discussion on the motivations of and conditions for joint venture formation from the perspective of transaction-cost theory.
} 
As opposed to market exchange, the formation of an alliance reduces transaction costs, as parties in alliances are expected to behave cooperatively as long as their rewards are largely dependent upon their own performance outcomes (e.g. Combs and Ketchen 1999). Therefore, the formation of alliances is expected to reduce the scope of opportunistic actions (which minimizes transaction costs) as well as to decrease the costs of production through joint coordination of the activity.

Nevertheless, since alliances are not immune to opportunistic behaviour of alliance partners, partner opportunism is found as the inherent source of instability in strategic alliances. Anticipating opportunism, partner firms negotiate and write contracts and use governance devices to monitor and address contractual breaches (Joskow, 1987) all of which accumulates in high transaction costs and endanger the outcome of the alliance (Das and Teng, 2000). To reduce the risks of opportunistic behaviour, transaction cost scholars advocate the formation of equity based joint ventures, where partner firms hold stakes in the alliance (Pisano, 1989; Pisano, Russo and Teece, 1988; Hennart, 1988). The ownership aspects of an equity-based joint venture are suggested to limit opportunistic behaviour as the partners are joined in their mutual interest to maintain the partnership (Jarillo, 1988; Osborn and Baughn, 1990). Asset specificity in an equity-based joint venture can further reduce the partners' incentive to engage in opportunistic behaviour, as both firms must invest in specific assets that lead to a reciprocal dependency (Dyer, 1996; Klein and Murphy 1988; Teece, 1987). This reciprocal dependency leads to a 'mutual hostage' situation through ex ante commitments by alliance partners and hence reduces the possibility of opportunistic behaviour over the course of the alliance (Pisano, 1989). Consequently, alliance research that has adopted a transaction cost perspective suggests that the key to successful alliances is the choice of appropriate contractual agreements that limit opportunistic actions of partner firms (Kogut, 1988).

\subsubsection{Knowledge based view: the importance of trust in learning alliances}

Drawing on knowledge-based theory (Nonaka and Takeuchi, 1995; Grant, 1996; Spender, 1996), alliances are suggested to be ideal platforms for learning, where 'knowledge creation occurs in the context of a community, one that is fluid and evolving rather than rightly bound or static' (Powell, Koput and Smith-Doerr 1996:118). Especially in knowledge intensive industries, strategic alliances are employed as the primary vehicle for accessing and 
sharing knowledge (including technology, know-how and organizational capabilities) across firms (Barringer and Harrison, 2000; Grant and BadenFuller, 2002; Hamel, 1991).

Within this field, the great majority of studies have assumed an 'organizational learning perspective', proposing that the main rationale of strategic alliances is to acquire the knowledge of the alliance partner. This rationale is closely related to what Spender (1992) called 'knowledge application' and what March (1991) referred to as 'exploitation'. In knowledge application alliances, the firm aims to "access the partner's stock of knowledge in order to exploit complementarities, but with the intention of maintaining its distinctive base of specialised knowledge" (Grant and Badenfuller, 2002: 421). In contrast, 'knowledge generation' (Spender 1992) or 'exploration' (March, 1991) points to alliances "as vehicles of learning, in which each member firm uses the alliance to transfer and absorb the partner's knowledge base" (Grant and Badenfuller, 2002: 421). In either case, these alliances allow partner firms to create a knowledge base that can present a crucial source of competitive advantage (Nonaka and Takeuchi, 1995; Grant, 1996; Spender, 1996). Nevertheless, learning alliances can easily result into 'learning races' where partner firms aim to 'outlearn' each other and then under invest after they achieved their learning objective (Hamel, 1991). Numerous studies warn again the detrimental effects of learning races on alliance outcomes (e.g. Khanna et al., 1998). This inherent risk of loosing core capabilities or skills to partner firms presents a considerable source of conflict that has evoked a large share of alliance research to highlight the importance of trust for securing the successful outcome of learning alliances (Ring and Van de Ven, 1992; Gulati, 1995; Zaheer, McEvily and Perrone, 1998). To minimize the threat of opportunistic behaviour in learning alliances, numerous scholars advocate the development of mutual trust to reduce the fear of opportunistic behaviour (Gulati, 1995; Zaheer et al., 1998).

Informed largely by social exchange theory (Homans, 1961; Blau, 1964), scholars emphasise the importance of relational factors for the performance of alliances (e.g. Muthusamy and White, 2005; Sarkar et al., 2001; Spekman et al., 2000; Doz, 1996; Gulati, 1995; Ring and Van de Ven, 1992). In particular, this research view challenges the logic of 'contractual based governance' in transaction cost theory, and promotes the importance of 'relational based 
governance' for attaining successful alliance outcomes. In particular, the relational view criticises the transaction cost perspective on alliances for its 'its singular focus on partner opportunism' (Muthusamy, White and Carr, 2007) and its failure to capture the important role of interfirm trust for alliance performance (Gulati, 1995). Within this 'relational view' of alliances, transaction costs are no longer minimized by the creation of a 'mutual hostage' situation but through the development of interorganizational trust (Gulati, 1995; Ring and Van de Ven, 1992; Zajac and Olsen, 1993). Defining trust in an alliance as the reliance on another party under conditions of risk (Nooteboom, 1996), trust is found to be an important characteristic for successful relational exchanges (Sivadas and Dwyer, 2000; Smith and Barclay, 1997; Weitz and Bradford, 1999). Another prominent building block for alliance success includes reciprocal commitment (Anderson and Weitz, 1992; Moorman et al., 1992) that induces a 'sense of duty to the venture and the other party' (Muthusamy and White, 2005). Spekman et al. (2000:43) even find that "without trust and commitment, there can be no alliance".

The important role of trust and commitment for alliance success has been particularly emphasised in the context of learning alliances (Arino and de la Torre, 1998; Lazaric, 1998), where the effectiveness of knowledge exchange depends on the degree of transparency between partner firms (Doz and Hamel, 1998) and suspicion and anticipation of opportunistic behaviour reduces the willingness to share knowledge. Further, mutual trust encourages partners to develop idiosyncratic knowledge-sharing routines that facilitate knowledge transfer and learning (Dyer and Singh, 1998; Kale et al., 2000). As opposed to relying on contractual based governance, the development of mutual trust promotes the formation of repeated partnerships $(\mathrm{Gu}-$ lati, 1995; Zaheer et al., 1998). Hence, trust is suggested to decrease uncertainty and enable the formation of loyal, cooperative and stable alliances (Morgan and Hunt, 1994; Hewett and Bearden, 2001). In similar vein, Kale, Singh and Perlmutter (2000) find the development of 'relational capital' (referring to the level of mutual trust, respect and friendship in an alliance relationship) to significantly curb opportunistic behaviour, facilitate learning and hence contribute to the successful conduct of learning alliances. 


\subsubsection{Resource-based view: the role of idiosyncratic and complementary resources}

A third stream of alliance research has largely adopted the logic of the resource-based view (Penrose, 1959; Barney, 1991; Nelson, 1991; Peteraf, 1993; Prahalad and Hamel, 1990) to research the rationales for strategic alliance formation and conditions for alliance success. The resource-based view conceptualises firms as bundles of resources ${ }^{5}$ and hence finds that a firm's competitive position is defined by its resource base (Das and Teng, 2000). ${ }^{6}$ Firms will enjoy superior financial performance when they have access to resources that are rare, inimitable, and nonsubstitutable. Establishing such a unique collection of resources allows firms to implement value-creating strategies that cannot be easily duplicated by competing firms (Barney, 1991; Nelson, 1991; Penrose, 1959; Peteraf, 1993; Prahalad and Hamel, 1990, Teece, Pisano and Shuen, 1997).

In the view of resource-based scholars, strategic alliances often provide a superior means to gaining access to desired resources as compared to market transactions or internalisation (e.g. mergers and acquisitions). First, given that resources are often intangible, tacit and difficult to price makes those infeasible to obtain on the spot market (Mowery et al 1996; Chi, 1994). Second, while mergers and acquisitions can present an efficient strategy to secure access by fully internalising the target firm's resources, firms often face the challenge that many undesired or unneeded resources are fused with needed resources. Hence, merging with or acquiring the entire target firm may result in a bulk of unneeded resources (Hennart and Reddy, 1997; Ramanathan, Seth and Thomas, 1997; Das and Teng, 2000). Especially, when a certain degree of asset specificity is involved, the attempt to get rid of the less valuable or redundant resources in an M\&A inevitably incurs high losses (Ramanathan et al., 1997). Therefore, in the case when non-desired resources are not easily separable from desirable resources, strategic alliances are suggested to be the preferred strategy.

\footnotetext{
${ }^{5}$ Resources are suggested to include physical, human or organizational assets as well more abstract notions such as machine capacity, customer loyalty or production experience (Wernerfelt 1984)

${ }^{6}$ The close relationship between firm resources and competitive advantage has been established by strategy literature; e.g. Reed and DeFillippi (1990)
} 
Alliances allow partner firms to retain their own corporate identities while efficiently accessing only the resources each desires while bypassing nondesired ones and thereby reducing superfluity. Consequently, drawing on the logic of resource-based theory, strategic alliances allow the 'resourceconstrained firm' (Oviatt and McDougall, 1994) to create a heterogeneous and unique resource base (Amit and Schoemaker, 1993; Mahoney and Pandian, 1992) that can become a viable source of competitive advantage and eventually lead to above-normal returns (Rumelt, Schendel and Teece, , 1984; Barney, 1986, Peteraf 1993). Therefore, strategic alliances are suggested to facilitate the pooling and combining of valuable resources that enables companies to achieve value maximisation (Eisenhardt and Schoonhoven, 1996; Gulati, 1999; Rothaermel, 2001; Van De Ven and Walker, 1984).

Especially in case where a firm is in a vulnerable strategic position needing resources or in strong social positions possessing valuable resources to share, the likelihood of strategic alliance formation increases (Eisenhardt and Schoonhoven (1996). Vulnerable strategic positions occur when firms pursue expensive or risky strategies and face changes in both structure and competitive dynamics. In such situations, alliance partners can provide critical resources and skills as well as legitimacy and market power (e.g. Weiwel and Hunter, 1985; Hagedoorn, 1993, Baum and Olivier 1991) that improve the strategic position of the 'vulnerable' firm. Hence, the underlying logic to enter strategic alliances for firms in vulnerable strategic positions is need. Contrary, firms that enjoy strong social positions due to their status and reputation are favoured alliance partners. Their extensive personal relationships create an awareness of opportunities for alliances and induce trust among potential partners (Eisenhardt and Schoonhoven 1996). In this regard, strategic alliances allow firms to protect vulnerable strategic positions or exploit strong social positions.

Given the 'quest for resources' as the driving motivation for alliance formation, it is not surprising that alliance success is suggested to be a function of the resources each partner contributes. Complementary and idiosyncratic resources are suggested to foster alliance success (Jap, 1999). We find a large share of alliance literature devoted to the performance implications of 'complementary resources' in strategic alliances where complementary resources are suggested to enhance the efficiency and effectiveness of the performance of alliance partners (e.g., Chung, 2000; Varadarajan and Cunningham, 1995; 
Bleeke and Ernst, 1991, Sarkar et. al. 2001). Further, the ability of the alliance to produce 'idiosyncratic resources' is suggested to define alliance success (Lambe et al., 2002). Idiosyncratic resources are developed during the lifetime of an alliance by combining and integrating the respective resources of the alliance partners (Jap, 1999). Following Das and Teng (2000), developing idiosyncratic resources creates "more value in the integrated condition than the sum of the separate values of the resources with individual firms" (p.49). To conclude, from resource-based perspective, the main determinant of alliance success is the presence of strategic fit in terms of complementary resources and the development of idiosyncratic resources (Sarkar et al., 2001; Harrison, Hitt, Hoskisson and Ireland, 1991; Hitt, Harrison and Ireland, 2001c; Ireland et al., 2001b).

\subsubsection{Social network theory}

The importance of accessing resource and sharing knowledge as driving forces of alliance formation is further elaborated by social network theory. From a social network perspective, the increased rate of network formation in many industries implies that resources and knowledge (i.e. technologies, know-how and organizational capabilities) are ever more embedded in interfirm networks (Gulati, 1999). Therefore, by entering into multiple alliance relationships simultaneously, the focal firm can access a larger quantity or volume of information and resources (Koka and Prescott, 2002). In addition to the benefits that accrue from a large quantity of information and resources, an alliance portfolio with diverse ties allows the focal firm to access different sources of information, knowledge or resources that it could not access by itself. Engaging with partner firms that possess different information and resources than the focal firm implies non-redundancy, that is, the contextual overlap of information context between the partner firms is kept at a minimum. Thus, non-redundancy implies the presence of structural holes between focal firm's partners (Burt, 1992). Therefore, creating a portfolio of diverse alliance relationships enables the focal firm to exploit structural holes between partner firms (Gulati, 1998).

Conversely, interfirm networks are characterised as redundant, if they offer access to the same information and resources (Hoffmann, 2007). In case interfirm relations are highly redundant, these may even prevent the focal firm from obtaining novel information critical to its competitive survival (Uzzi, 
1996, 1997). Therefore, Baum, Calabrese and Silverman (2000) warn against increasing the number of alliances without considering partner diversity, as this would yield an inefficient configuration with little diverse information. Similarly, Goerzen and Beamish (2005) state that firms with diverse ties are exposed to a variety of ideas and perspectives that firms with primarily redundant contacts cannot enjoy. Hence, increasing the diversity of interfirm ties increases the scale and scope of available information and resources to the focal firm. As a result, the focal firm accumulates social capital.

In line with social network theory, a firm's alliance portfolio corresponds to its social capital (Koka and Prescott, 2002), meaning access to greater network resources (Chung, Singh and Lee, 2000). Social or relational capital develops through long-term interaction between strategic alliance partners (Kale, Singh and Perlmutter, 2000) and is positively related to the extent of resource exchange between organizations (Tsai and Ghoshal 1998). Therefore, creating a portfolio of alliances does not only give access to the resource base of a partner firm but to its' networks.

The importance of social capital for alliance portfolio creation is further emphasised by the relational embeddedness theory, which postulates that the joint history of collaborations between partner firms increases social capital and trust, inducing additional alliances between the partner firms (Goerzen, 2007, Gulati, 1995, Walker, Kogut and Shan, 1997). Similarly, the structural embeddedness perspective argues that prior indirect alliance ties induce collaboration between the focal firm and potential new alliance partners, hence providing a rationale to add alliances to the portfolio (Gulati and Garguilo, 1999). Consequently, from a network and social capital theory perspective it follows that 'the greater the diversity of alliances maintained, the richer would be the benefits of the firm's portfolio' (Goerzen and Beamish 2005).

Following Kogut (2000), the configuration of alliance portfolios can give rise to two different forms of relational rents. Depending whether a firm's alliance portfolio consists of redundant strong ties to similarly positioned alliance partners or nonredundant weak ties to partners outside its familiar business domain influences the type of relational rent that a firm can enjoy. When the alliance portfolio consists mainly of redundant strong ties, the firm has built relatively stable alliances based on trust and hence can attain the so called 'Coleman rent' (Kogut, 2000). On the other extreme, when the 
portfolio consists mainly of alliances with unfamiliar firms (nonredundant weak ties) then the focal firm has the opportunity to exploit structural holes and hence benefit form the so called 'Burt rent' (Kogut, 2000).

Surely, both cases are extreme. The point is to illustrate that designing a portfolio of alliances will always involve a certain trade-off between the richness of information and resources obtained from similar partner firms (strong and redundant ties) and, on the other hand, the diversity of information and resources obtained from dissimilar firms (weak and nonredundant ties). Engaging in strong ties will stabilise the alliance portfolio, while weak ties ensure the flexibility of the alliance portfolio - a trade-off that certainly needs careful attention paid by alliance managers. Consequently, the logic of network and relational view theory implies that inter-firm ties that provide the focal firm with access to diverse network resources can create relational rent and serve as an invaluable source of competitive advantage (Dyer and Singh, 1998; Gulati et al., 2000).

\subsubsection{Dynamic capability view of the firm: the role of alliance capability}

A large share of recent alliance studies has drawn upon the dynamic capability view of the firm to explain the conditions for alliance success and to derive what has become termed the alliance capability view. Introduced in the 1980s, the dynamic capability view answers the question how firms achieve sustainable competitive advantage. Extending the theoretical logic of the resource based view, the dynamic capability view suggests that resource endowment is not sufficient to cause performance differences among firms. In particular, resource-based theory seems to assume that these valuecreating resources simply 'exist', regardless of the firm's changing business environment that might quickly erode the firm's competitive advantage. One important question that remains unanswered in this context is how are these resources developed, integrated and released? To sustain the firm's competitive advantage, dynamic capability scholars emphasise that firms do not operate in static environments but need to adjust and renew their resource bases to match the changing demands of their environment. Hence, while resources are the input (tangible or intangible) to production that a firm owns and controls, an organizational capability "refers to the ability of an organization to perform a coordinated set of tasks, utilizing resources, for the purpose of achieving a particular end result" (Helfat and Peteraf, 2003:999). That is, while resources (physical, human, organizational) are 
available to all firms, the 'capability' to deploy and alter them productively is heterogeneously distributed among firms. By altering the resource base acquiring new resources or recombining existing ones - companies are able to generate new value creating strategies (Grant, 1996; Pisano, 1994; Henderson and Cockburn, 1994; Teece et al., 1997). Therefore, dynamic capability scholars stress the necessity for firms to build 'higher-order resources' or capabilities that improve the productivity of 'basic' resources (Makadok, 2001).

Important to note, scholars draw a vital distinction between operational and dynamic capabilities. Following Helfat and Peteraf (2003:999) an "operational capability generally involves performing an activity, such as manufacturing a particular product" while "dynamic capabilities (...) do not involve production of a good or provision of a marketable service. Instead, dynamic capabilities build, integrate, or reconfigure operational capabilities". Both, operational and dynamic capabilities are suggested to consist of "routines to execute and coordinate the variety of tasks required to perform the activity" (Helfat and Peteraf, 2003:999). Herewith the authors refer to Nelson and Winter's (1982: 97) definition of routines as 'repetitive, highly patterned activities or behaviour that are learned'. According to Nelson and Winter (1982), skills are embodied in individuals, while routines are found the organizational level where they act as 'rules of conduct' or 'best practices' for the members of the organization. The notion of routines is crucial for the definition of capabilities (operational or dynamic) as routines imply that the organization must have reached some threshold level of practiced activity. As Winter (2003) stated, 'brilliant improvisation is not a routine'.

The related notion of 'organizational competence' is often used synonymously for the notion of dynamic capability. According to Sanchez et al. (1996:8), a competence is the firm's ability to "sustain the coordinated deployment of assets in a way that helps a firm to achieve its goals". Similar to the definition of capabilities, a competence is defined to be a higher-order resource. Since for the purpose of this dissertation a thorough differentiation between capabilities and competences is not vital, we use the term capability to denote a firm's collection of routines that enable it to execute and coordinate the variety of tasks required to perform an activity (Helfat and Peteraf, 
2003:999)7. What is essential is the argument that differences in organizational capabilities between firms are suggested to explain the difference in firm performances (Lado, Boyd, and Wright, 1992).

The dynamic capability view has had several important implications for research on alliances. First, it promoted a fundamental shift in research focus. Whereas traditional alliance literature investigated critical aspects peculiar to the individual alliance relationship at hand, recent alliance research examined the firm as its unit of analysis and with it the firm's managerial capabilities as key to the firm's ability to create successful alliances. Therefore, in the view of alliance capability scholars, the source of alliance success may not only lie in the relationship between the partners, but in the partners' ability to manage alliance relationships. Consequently, alliance capability scholars suggest that the difference in alliance performance rates between firms can be explained by the firms' organizational capability to manage alliances. This 'alliance capability' or 'alliance competence' (as it used interchangeably in alliance literature) is suggested to entail different aspects related to the formation, management and termination of alliances. For instance, Lambe et al (2002:145) proposed that alliances are successful when the partners have developed the ability for securing, developing and managing alliances.

To put it boldly, not the relationship is studied, but the ability of the individual partners to manage the relationship. Figure 2.1 shows the differences in focus between traditional alliance research and research into alliance capability.

With this shift in focus, new factors have been introduced to explain alliance success. Alliance capability studies emphasise the importance of accumulating alliance experience for attaining successful alliance outcomes (Shan et al., 1994; Anand and Khanna, 2000; Kale et al., 2002; Heimeriks and Duysters, 2007). By engaging in numerous alliances, and hence accumulating experience, firms learn the crucial aspects of alliance management e.g. recognizing potential pitfalls and troublesome situations in the collaboration and hereby

\footnotetext{
${ }^{7}$ For a detailed discussion of the differences between these notions, we refer to Sanchez et al. (1996).
} 
increasing the chances to manage alliances successfully on a continuous basis (Anand and Khanna, 2000).

Figure 2.1: Alliance capability research versus traditional research

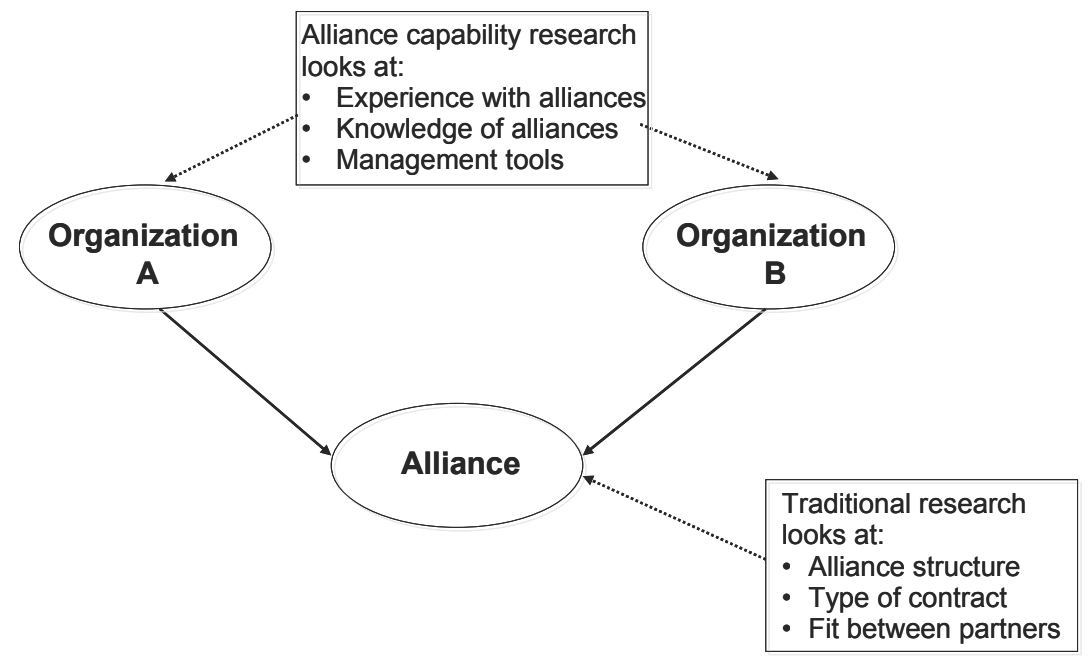

Source: Draulans et al. (1999)

Moreover, studies emphasise the need for managerial practices that guide and support the alliance management on a daily basis. Examples of alliance management practices that are found to raise the performance levels of a firm's alliances are alliance management training programmes (Draulans et al., 2003), the introduction of an alliance department coordinating all alliance-relevant activities (Borker et al., 2004) or the use of an alliance database that disseminates alliance relevant knowledge throughout the firm (Harbison and Pekar, 1998). It follows that some firms are more capable of collaborating than others, due their level of experience, the knowledge they have acquired about alliance management and the presence of effective management processes related to alliances.

For practice, this shift is important as well. Rather than the traditional advice given to managers about looking for strategic fit with a partner or choosing the right governance structure, advice based on the alliance capability view would revolve around investing in alliance managers, in alliance management tools and in gaining knowledge about alliances. The notion of alliance capability implies an organization-wide ability to manage alliances as opposed 
to alliance expertise residing in individuals inside the firm. To illustrate, firms that have developed alliance capability are characterised by having an institutionalised approach to learning about alliance management, they have standardised managerial procedures and have often formed a dedicated staff with a high degree of experience and expertise sharing that allows for the development of some repository of knowledge for future use (Harbison and Pekar, 1997:83).

In this regard, firms that possess alliance capability are different from firms that depend on a trial-and-error approach to alliance management with no best practices or expertise to rely on. This ad-hoc approach captures no lessons for future alliances and often results in unsatisfactory outcomes. Firms without alliance capability may rely on alliance specialists, but since no sharing of expertise takes place, the alliance knowledge residing in these individuals is lost when the specialists leave the firm. Therefore, alliance capability is suggested to be an organization-wide ability of the firm to manage its alliances. The value creating potential of alliance capability stems from the fact - that similar to other organizational capabilities - the proficiency to manage alliances effectively is firm specific and hence impossible for other firms to buy or imitate (Leonard Barton, 1992; Teece and Pisano, 1994; Henderson and Cockburn, 1994; Eisenhardt and Martin, 2000). Therefore, developing alliance capability can serve as an invaluable source of competitive advantage

Following this logic, scholars posit that developing alliance capability enables firms to master the difficult task of alliance management (Ireland et al., 2002) and hence is considered as the main determinant of alliance performance (Kale and Singh, 1999; Sanchez, 2001; Draulans et al., 2003; Anand and Khanna, 2000; Leonard-Barton, 1992; Henderson and Cockburn, 1994; Eisenhardt and Martin, 2000).

\subsection{Discussion and conclusions}

Without doubt, each of these presented streams of alliance research have considerably influenced our understanding of alliance formation rationales and provided critical grounds for further investigating the phenomenon of strategic alliances. However, we find that not all of the presented theories can quite capture the recent developments in the field of strategic alliances. 
In particular, the current trend towards building portfolios of alliances calls for a critical reflection on the appropriateness of theories for explaining alliance portfolio formation and success factors.

\subsubsection{How well do these theories capture the importance of building alliance portfo- lios?}

Alliance research that adopts a transaction costs view has been severely criticised to confine the rationale for strategic alliance formation to efficiency and cost-minimization factors only (Barringer and Harrison, 2000: Faulkner, 1995). In the view of transaction costs theorists, firms are treated as individual, self-fulfilling units that prefer 'going alone' to collaboration (Williamson, 1975, 1991). In this regard, alliances can only be considered as a secondbest option, an alternative to market transaction or internalisation. Therefore, in our view, depicting alliances as mere cost minimizing governance devices does not seem to take into account the 'unsettling new reality' that includes the globalization of markets, the search for new capabilities, and intensifying competition for markets" (Harbison and Pekar, 1998:11). Faced with this reality, firms can no longer rely on the traditional mode of selfsufficiency and closed innovation as propagated by transaction costs scholars. Neither can firms rely on a single high profile alliance to fulfil their goals, but increasingly need to depend on various external partners to enhance their resource endowment (Eisenhardt and Schoonhoven, 1996) and share technology and knowledge (Dyer and Nobeoka, 2000; Kale, Singh, Perlmutter, 2000). Therefore, it seems difficult to comprehend the phenomenon of building large alliance portfolio from a transaction cost perspective. In contrast, the logic of resource-based, knowledge-based and social network theory commonly emphasise the importance of accessing a variety of valuable resources and knowledge as underlying drivers of firm competitiveness and thus promote the creation of large and complex alliance portfolios, which allow firms to access a large quantity of different resources and knowledge. Therefore, we find these three theories particularly insightful into why firms build portfolios of alliances.

The alliance capability view does not explicitly provide a rationale for firms to build alliance portfolios. Nevertheless, its underlying logic implies the importance of accumulating alliance experience for improving the firm's proficiency in managing alliances. Alliance capability literature often con- 
ceptualised a firm's alliance portfolio as a "repository of experience as well as a vehicle for learning" (Wassmer, 2008: 18). By allying with a large number of different partners, the firm gains experience about the crucial aspects of alliance management and increases its chances to mange alliances successfully. Therefore, from an alliance capability view, engaging in multiple alliances simultaneously enhances the firm's development of alliance capability (which in turn, is associated with high alliance success rates).

\subsubsection{How well do these theories explain success factors of alliance portfolios?}

Theories such as transaction cost, resource based and knowledge-based view of the firm commonly put a strong emphasis on either relational factors (e.g. trust and commitment) or structural factors (choice of governance, strategic fit in terms of resources). These factors are collectively referred to as relational advantage (Dyer and Singh, 1998) or collaborative advantage (Kanter, 1994) and have been suggested to explain the collaboration-specific rent (Madhok and Tallman, 1998) that companies enjoy from an alliance. Thus, these theories do well in explaining success factors for individual alliances, but cannot explain sustained success of firms on the alliance portfolio level.

Regarding social network studies on alliance portfolio success factors, we find a strong emphasis on the structural characteristics of the alliance portfolio as the main determinant for alliance portfolio success. This literature provides no further insight into how firms can attain successful alliance portfolio configurations, that is, how do firms know or learn about effective portfolio configuration and how they can learn to ensure that each alliance relationship is contributing to the overall goal of the alliance portfolio.

In contrast, the alliance capability view does explicitly contribute to the explanation of why some firms are more able to sustain successful alliance portfolios than other firms. Directing the research focus to the internal organization of the firm, the development of an organization-wide alliance capability to manage alliances is found to be the key determinant for alliance success. Rather than the traditional advice given to firms to ensure strategic or cultural fit with a partner or choosing the right governance structure, advice based on the alliance capability view emphasises the importance of accumulating valuable knowledge about alliance management and investing in effective alliance management practices that can be applied across the portfolio of a firm's alliances. 
Critically evaluating these different theoretical perspectives, we conclude that none of these theories provides a comprehensive answer to both of our questions. Instead, each theory offers indispensable insight for either alliance portfolio formation rationales or alliance portfolio success factors. Thus, for the course of this dissertation, we refer to various theories in order to understand the motivations behind creating a portfolio of alliance, sources of instability and critical success factors.

An important message of social network studies on alliance portfolios is the understanding that 'going alone' is no longer a viable option for the majority of companies: creating a portfolio of alliances allows partner firms to perform activities together that neither could perform alone. Therefore, we will refer to social network theory in order to explain the phenomenon of alliance portfolios.

Acknowledging alliance capability as the main determinant of alliance success implies that companies are no longer dependent upon the structural characteristics of the alliance relationship at hand but can consistently attain high alliance performance rates across their alliance portfolio by actively investing into and advancing their proficiency in alliance management. In order to understand how firms can build alliance capability, we draw on dynamic capability view, knowledge-based view, organisational learning theory and evolutionary economics. Therefore, combining the essential arguments of both social network and alliance capability view allows us to address the important alliance portfolio relevant issue of building alliance capability for superior alliance portfolio performance. 



\section{Chapter 3 Alliance CAPAbility: AN Elusive PHENOMENON}

\subsection{Introduction}

Over the last decades, various schools of thought contributed to the unravelling of critical success factors for alliance performance. In Chapter 2 we witnessed a shift in the research focus from an emphasis on relational (e.g. cultural fit, trust) and structural factors (e.g. choice of governance) towards a focus on a firms' collaborative skills or so-called alliance capability. We reviewed how the alliance capability view has extended the logic of the dynamic capability view to explain the heterogeneity in alliance performance rates among firms. Alliance capability studies claim that the source of alliance success may not only lie in the relationship between the partners, but in the internal organization of each of the individual partners as well. Some companies may be more capable at collaborating than others, because they have more experience, knowledge or management processes related to alliances. Numerous studies confirm that firms that have developed alliance capability continuously outperform firms without alliance capability (Kale and Singh, 1999; Sanchez, 2001; Draulans et al., 2003; Anand and Khanna, 2000; Leonard-Barton, 1992; Henderson and Cockburn, 1994). Therefore, as discussed in the previous chapter, we have decided to adopt an alliance capability view to studying how firms can attain successful alliances and alliance portfolios.

The purpose of this chapter is to clarify the notion of alliance capability and its organisational determinants. This is vital, as extant conceptual work on alliance capability is plagued with inconsistencies and ambiguities, as it is reflected by the numerous terms used and various definitions provided. In particular, alliance literature seems to disagree about what elements specifically constitute alliance capability (Schreiner et al., 2009). As a result, we find no single clear-cut definition of alliance capability. 
To address this shortcoming, we review and critically discuss extant alliance capability literature and derive an own definition of alliance capability that reflects the multi-dimensional construct of this notion. Second, we clarify how firms can develop alliance capability. Reviewing state-of-the-art literature, we note a widespread agreement in alliance literature that accumulating alliance experience is decisive for the development of alliance capability (e.g. Anand and Khanna, 2000; Hoang and Rothaermel, 2005). Some scholars thereby suggested that in order to build alliance capability and to capitalize on its accumulated alliance experience, the firm needs to develop organisational routines to capture, internalise, and apply alliance experience in the form of accessible knowledge throughout the organization (Kale et al. 2002; Kale and Singh, 2007). Alliance literature is filled with examples of so-called 'learning routines', 'processes', 'mechanisms' and 'management practices' for alliance capability building. The sheer volume of examples accompanied by ambiguities and inconsistencies in terminology leads to a fragmented understanding of the organisational determinants of alliance capability. Therefore, by reviewing extant literature we delineate the different types of organisational determinants that are necessary to build alliance capability.

In the first part of this chapter, we review state-of-the-art literature on the subject of alliance capability and propose an own definition of alliance capability. In the second part of this chapter, we review how alliance studies have drawn on various theories to explain alliance capability building. We conclude with an overview of organisational determinants of alliance capability and classify these according to their main purpose in the capability building process.

\subsection{What is alliance capability?}

\subsubsection{Concepts and definitions of alliance capability in extant alliance literature}

The first efforts to explain alliance performance from an alliance capability view emerged in the 1990s. Over time, the definition of the concept of alliance capability evolved. Table 3.1 provides a chronological overview of concepts referring to alliance capability. The first time the literature discussed the issue of alliance capability was in 1993 when Huxham (1993) dubbed the term collaborative capability. 
However, it appears that other researchers did not pick up this topic as none of the later authors refer to this paper (De Man, Duysters and Saebi, 2010). Subsequent work on the concept of alliance capability was brought forward by consultants Harbison and Pekàr (1997), discussing the issue of alliance capability in a white paper and provided some first, albeit crude, empirical evidence that firms that invest more in alliance management skills are more successful with alliances than companies that do not make such investments. In the same year, on a more academic note, Simonin (1997) published results of a large-scale research showing that collaborative know-how is a determinant of alliance success. Successively, Draulans et al. (1999) provided a more detailed statistical analysis of the effect of investments in alliance management tools (e.g. alliance management training, alliance evaluation techniques) on alliance success. A more refined version of this study was published four years later (Draulans et al., 2003) and was taken up and extended by later authors (e.g. Duysters and De Man, 2007; Heimeriks and Duysters, 2007).

In parallel, Spekman and Isabella (2000) referred to the notion of 'alliance competence', highlighting the important role of 'know how', supporting processes and structure, mindset, learning and bench depth (having sufficient alliance staff) as part of a firm's competency in alliance management. Consecutively, alliance competence has been defined as the "organizational ability for finding, developing, and managing alliances" (Lambe et al 2002: 142). Independently, Kale et al. (2001) defined the concept of alliance skills and later, alliance capability (Kale et al., 2007).

By then, more authors had identified alliance capability as a relevant theme (see table 3.1) and the diverse streams of literature appear to gradually connect into a more coherent research agenda. 
Table 3.1: Alliance capability and related concepts in chronological order

\begin{tabular}{|c|c|c|}
\hline Author & Concept & Definition \\
\hline Huxham (1993, p. 23) & $\begin{array}{l}\text { Collaborative } \\
\text { capability }\end{array}$ & $\begin{array}{l}\text { "...the capacity and readiness of an organization } \\
\text { to collaborate" }\end{array}$ \\
\hline $\begin{array}{l}\text { Harbison \& Pekàr } \\
\text { (1997) }\end{array}$ & $\begin{array}{l}\text { Alliance capa- } \\
\text { bility }\end{array}$ & undefined \\
\hline Simonin (1997) & $\begin{array}{l}\text { Collaborative } \\
\text { know-how }\end{array}$ & $\begin{array}{l}\text { skill in identifying, negotiating, managing, moni- } \\
\text { toring and terminating collaboration }\end{array}$ \\
\hline $\begin{array}{l}\text { Draulans et al. (1999, } \\
\text { p. 53) }\end{array}$ & $\begin{array}{l}\text { Alliance capa- } \\
\text { bility }\end{array}$ & "...the ability to manage alliances successfully" \\
\hline Gulati (1999: 402) & $\begin{array}{l}\text { Alliance forma- } \\
\text { tion capability }\end{array}$ & $\begin{array}{l}\text { "...organizational capabilities that enable firms to } \\
\text { form alliances with greater ease". }\end{array}$ \\
\hline $\begin{array}{l}\text { Anand \& Khanna } \\
(2000: 295)\end{array}$ & $\begin{array}{l}\text { Alliance capa- } \\
\text { bility }\end{array}$ & "...ability $(\ldots)$ to create value through alliances" \\
\hline $\begin{array}{l}\text { Spekman and Isabella } \\
(2000)\end{array}$ & $\begin{array}{l}\text { Alliance com- } \\
\text { petence }\end{array}$ & $\begin{array}{l}\text { a function of individual skills, capabilities, and } \\
\text { firm-level attributes that enhance, encourage and } \\
\text { support alliance-like thinking and behaviour } \\
\text { throughout the firm }\end{array}$ \\
\hline $\begin{array}{l}\text { Kale et al. (2001, p. } \\
464) \text {, }\end{array}$ & Alliance skills & $\begin{array}{l}\text { "Alliance skills, coordinate, capture, codify, com- } \\
\text { municate and create alliance management lessons } \\
\text { and insights associated with alliance experience" }\end{array}$ \\
\hline $\begin{array}{l}\text { Lambe, Spekman \& } \\
\text { Hunt (2002, p.142) }\end{array}$ & $\begin{array}{l}\text { Alliance com- } \\
\text { petence }\end{array}$ & $\begin{array}{l}\text { "the organizational ability for finding, developing } \\
\text { and managing alliances" }\end{array}$ \\
\hline $\begin{array}{l}\text { Kale, Dyer and Singh } \\
(2002, \text { p. } 750)\end{array}$ & $\begin{array}{l}\text { Alliance capa- } \\
\text { bility }\end{array}$ & $\begin{array}{l}\text { The firm's ability to effectively "capture, share } \\
\text { and disseminate the alliance management know- } \\
\text { how, associated with prior experience" }\end{array}$ \\
\hline $\begin{array}{l}\text { Draulans et al. (2003, } \\
\text { p. 152), }\end{array}$ & $\begin{array}{l}\text { Alliance capa- } \\
\text { bility }\end{array}$ & $\begin{array}{l}\text { "...the ability to create successful alliances, based } \\
\text { on learning about alliance management and lever- } \\
\text { aging alliance knowledge inside the company" }\end{array}$ \\
\hline $\begin{array}{l}\text { Rothaermel \& Deeds } \\
(2006, \text { p.431) }\end{array}$ & $\begin{array}{l}\text { Alliance man- } \\
\text { agement capa- } \\
\text { bility }\end{array}$ & $\begin{array}{l}\text { "A firm's ability to effectively manage multiple } \\
\text { alliances" }\end{array}$ \\
\hline $\begin{array}{l}\text { Heimeriks, Duysters } \\
\text { \& Vanhaverbeke } \\
(2007, \text { p.374) }\end{array}$ & $\begin{array}{l}\text { Alliance capa- } \\
\text { bility }\end{array}$ & $\begin{array}{l}\text { "...a higher-order resource that is difficult to } \\
\text { obtain or imitate and has the potential to enhance } \\
\text { the performance of the firm's alliance portfolio" }\end{array}$ \\
\hline $\begin{array}{l}\text { Schreiner et al. (2009: } \\
\text { 1402) }\end{array}$ & $\begin{array}{l}\text { Alliance man- } \\
\text { agement capa- } \\
\text { bility }\end{array}$ & $\begin{array}{l}\text { "...alliance management capability as comprising } \\
\text { coordination, communication, and bonding skills } \\
\text { that are critical during the post-formation phase of } \\
\text { an alliance" }\end{array}$ \\
\hline
\end{tabular}

Overall, these studies have in common the essential idea that companies can develop an organizational capability that is responsible for the effective management of alliances. 
As the literature on alliance capability is an extension of the dynamic capability theory (please see Chapter 2) alliance capability scholars commonly agree that the value creating potential of alliance capability stems from the fact that the proficiency to manage alliances effectively is firm specific and hence impossible for other firms to buy or imitate. Moreover, a common theme to definitions in table 3.1 is the emphasis on alliance capability as an organisation-wide proficiency in managing alliances as opposed to alliance expertise residing in individuals inside the firm. To illustrate, firms that have developed alliance capability are characterised by having an institutionalised approach to learning about alliance management, they have standardised managerial procedures and have often formed a dedicated staff with a high degree of experience and expertise sharing that allows for the development of some repository of knowledge for future use (Harbison and Pekar, 1997:83).

In this regard, firms that possess alliance capability are different from firms that depend on a trial-and-error approach to alliance management with no best practices or expertise to rely on. This trial-and-error approach captures no lessons for future alliances and often results in unsatisfactory outcomes. Hence, the notion of alliance capability in extant studies commonly refers to an organisation-wide dedication to alliance management by accumulating alliance management expertise as well as by developing standardised processes, which allow the firms to manage its alliances effectively.

However, we must note a number of critical shortcomings in extant definitions of alliance capability. First, we would like to draw the attention to a suggested analogy between alliance capability and alliance success. To illustrate, Draulans et al $(1999,2003)$ refer to alliance capability as the ability to manage alliances successfully. Similarly, Anand and Khanna (2000:295) define alliance capability as "the ability to create value through alliances", while Rothaermel and Deeds (2006, p.431) define alliance capability as the "firm's ability to effectively manage multiple alliances". Besides the apparent tautology in defining capability as ability, these definitions incorporate the notions of effectiveness and success into the definition of alliance capability. By equalising alliance capability with superior performance, it seems as if scholars identify the presence of alliance capability ex post, inferring its existence from successful alliance outcomes. However, we argue that while superior performance of firms can be explained by the presence of alliance 
capability, the presence of alliance capability does not necessarily have to lead to higher performance. For instance, Heimeriks, Duysters and Vanhaverbeke (2007, p.374) define alliance capability more cautiously by pointing out that it is "a higher-order resource that is difficult to obtain or imitate and has the potential to enhance the performance of the firm's alliance portfolio". Hereby Heimeriks et al (2007) not only avoid the apparent tautology of defining alliance capability as 'ability' but emphasise the potential of alliance capability to enhance the performance of the firm's alliance portfolio, which implies that alliance success is not an inevitable consequence of alliance capability. Nevertheless, the definition by Heimeriks et al (2007) does not specify what constitutes alliance capability

Furthermore, we must note that there is no single clear-cut definition of alliance capability. In particular, we find that authors stress either one aspect of the alliance capability construct. On the one hand, some scholars define alliance capability as the mechanisms and routines to accumulate, store and disseminate alliance management know-how associated with prior experience (e.g. Draulans et al, 2003; Kale et al. 2001, 2002) hence referring to alliance capability as a learning capability of alliance management (Heimeriks and Duysters, 2007). As Sarkar et al. (2009) punctuate, these studies seem to define alliance capability in terms of its underlying organisational determinants.

On the other hand, some studies define alliance capability in terms of the skills needed to address important alliance management relevant tasks. For instance, Schreiner et al. (2009) define alliance capability as the 'coordination, communication, and bonding skills that are critical during the postformation phase of an alliance'. Another example is Simon' (1997) definition of alliance capability as the 'skill in identifying, negotiating, managing, monitoring and terminating collaboration', thus emphasising what constitutes alliance capability. Similarly, Lambe, Spekman and Hunt (2002, p.142) define alliance capability as the "organisational ability in finding, developing and managing alliances". Hence, while the first type of definitions indicate the underlying learning processes that enable firms to build alliance management expertise, the second group of definitions addresses tasks of alliance management.

Scholars such as Sarkar et al. (2009) and Schreiner et al. (2009) advocate a strict differentiation between what skills constitutes alliance capability and 
what underlies its development when defining alliance capability. However, we argue that a definition of alliance capability needs to integrate both the organisational determinants of alliance capability (i.e. learning processes to develop alliance capability) as well as indicating the tasks of alliance management (e.g. finding partners, coordination, monitoring etc.). We suggest that firms cannot address tasks of alliance management without having accumulated alliance management expertise neither is learning sufficient without identifying the tasks of alliance management. Therefore, we intend to derive a definition of alliance capability that incorporates both its underlying organisational determinants and indicates its tasks in alliance management. To this end, we will discuss alliance literature on these two issues and consecutively derive our own definition.

\subsubsection{Tasks of alliance management}

In general, alliance capability literature has identified constituents of alliance capability in terms of the skills needed to address important areas of alliance management as the alliance relationship passes through the various stages of its life cycle. Typically, these studies refer to challenges and tasks that arise as at different stages in the course of the alliance lifecycle (e.g. Child and Faulkner, 1998; Doz and Hamel, 1998). Figure 3.1 illustrates a simplified overview of the different phases involved.

Figure 3.1: Life cycle of alliance relationships

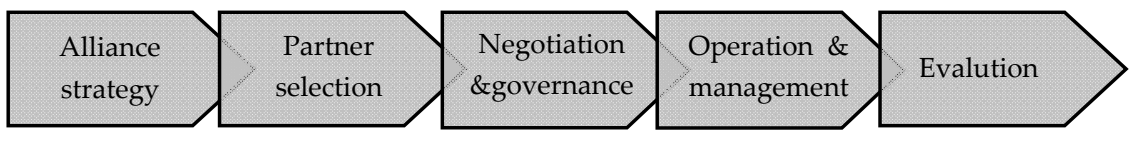

Source: adapted from Dyer, Kale and Singh (2001) and Das and Teng (1997)

In short, the first stage of the alliance life cycle generally refers to the development of an alliance strategy that sets the goals of the alliance at hand. The second stage involves the screening and selection of alliance partners, where both, cooperative and business compatibility, are assessed in depth. The third stage of the alliance life cycle evolves around the negotiation of the alliance contract, e.g. deciding upon mutual contributions in terms of financial or technological investments as well as the choice of governance structure. The fourth stage addresses daily operations of the alliance and requires 
the coordination and distribution of alliance tasks and responsibilities among alliance staff. Finally, as the alliance relationship evolves to the fifth stage, the alliance relationship has to be evaluated in terms of goal accomplishment and whether the relationship is worthwhile to continue, renegotiate, or to be terminated. Notably, studies differ on the terminology and number of these life cycle stages. Nevertheless, the description of these stages appears quite similar. ${ }^{8}$

In an attempt to define the constituent elements of alliance capability, alliance scholars proposed various skills that are crucial in order to manage the alliance as it passes through the stages of the lifecycle. For instance, referring to the different stages of the alliance lifecycle, Simonin (1997) defines alliance capability as the skills in identifying, negotiating, monitoring and terminating collaboration'. Similarly, Lambe et al. (2002) defines skills in 'finding, developing and managing alliances' to constitute alliance capability.

Furthermore, Gulati (1999: 402) defines alliance capability as the ability of firms to 'form alliances with greater ease' hence merely emphasising the formation phase of the alliance life cycle. Moreover, Schreiner et al. (2009) conceptualise alliance capability in terms of the skills that are critical in the post formation phase of the alliance relationship and thus omitting the formation and governance aspects of alliance management. Schreiner et al. (2009) suggest coordination, communication and bonding skills to constitute alliance capability as these skills are crucial in addressing the issues of coordination failures, communication lapses and underdeveloped personal bonds that potentially arise in any alliance relationship.

Critically reflecting on these definitions, we note that some of these appear to concentrate on a certain stage of the alliance lifecycle only; e.g. the formation stage as proposed by Gulati (1999) or the post-formation phase as proposed by Schreiner et al. (2009).

8 For instance, Dyer et al. (2001) identify the following five phases - alliance business case, partner assessment and selection, negotiation and governance, management, assessment and termination. Das and Teng (1997) who specify alliance strategy, partner selection, negotiation and formation, operation and finally evaluation and modification as the five stages of any alliance relationship provide a similar differentiation. Another example with four stages only is given by Spekman et al. (1996) who differentiate between anticipation, engagement and valuation, coordination and investment, and stabilisation and decision. 
By only focussing on the skills necessary for a particular stage in the alliance life cycle, these definitions provide a limited indication of the constituent elements of alliance capability. Furthermore, we like to add that Schreiner' et al. (2009) emphasis on coordination, communication and bonding skills appear to solely address challenges that might arise during the operation phase of the alliance; thus, they support neither the formation phase of the alliance nor the evaluation and termination phase of the relationship. We argue that the challenges and tasks that arise during all the stages of the life cycle are equally important and need to be addressed effectively.

The definition by Lambe et al. (2002) suffers from the fact that 'management of alliances' is included into the definition of alliance capability as a separate skill. However, we would like to point out that firms develop alliance capability in order to manage their alliances effectively. Therefore, alliance management should not be regarded as a separate skill but as the overarching aim of alliance capability. Hence, a definition of alliance capability needs to specify which skills are crucial to the management of alliances as the alliance passes through its life cycle. In this respect, the definition by Simonin (1997) appears to be clearest in terms of specifying the skills that are crucial to alliance management and thus constitute alliance capability.

In concluding what constituted alliance capability, we would like to boil down the various stages of the alliance lifecycle into the three phases only, namely, formation, operation and evaluation. We propose that a formation phase comprises the setting up of an alliance strategy, partner selection and negotiating of the alliance deal, while the operation and evaluation phases correspond to the initial phases in figure 3.1. Consequently, we delineate that alliance capability needs to address the formation, operation and evaluation of alliance relationships.

\subsubsection{The organisational determinants of alliance capability}

Some of the definitions in table 3.1 do not refer to the tasks that alliance capability needs to address, but indicate the underlying learning processes that enable firms to build alliance capability. For instance Kale et al. (2002, p.750) define alliance capability as the firm's ability to effectively "capture, share and disseminate the alliance management know-how, associated with prior experience". Similarly, Draulans et al. (2003, p. 152) define alliance capability as "the ability to create successful alliances, based on learning about alliance 
management and leveraging alliance knowledge inside the company". Herewith these definitions refer to alliance capability as a learning capability of alliance management. Two important issues are highlighted by these definitions, namely prior experience in managing alliances as well as the firm's ability to transform this experience into accessible lessons on alliance management that can be shared and disseminated throughout the organisation. Herewith these definitions refer to theoretical underpinnings of organisational capability development. ${ }^{9}$

Alliance experience is defined as the expertise on alliances generated through prior alliances (Gulati, 1995; Kale et al. 2002). By engaging in numerous strategic alliances, firms learn the crucial aspects of alliance management; e.g. recognizing potential pitfalls and troublesome situations in the collaboration (Anand and Khanna, 2000; Heimeriks and Duysters, 2007). As a result, experience is understood as repeated learning from practice over time. Accumulating alliance experience is therefore suggested to be a key determinant in alliance capability building. ${ }^{10}$

While acknowledging the important role of alliance experience, other scholars emphasise the importance of learning mechanisms for alliance capability building. As Kale et al. (2002) remarks, alliance experience is a necessary but not sufficient condition for alliance capability building as alliance experience may have little impact unless the tacit experience is internalised into accessible lessons on alliance management (Kale et al., 2002).

Rather, by the use of learning mechanisms, firms are able to capture, share and disseminate alliance management know-how, associated with prior experience. Therefore, a firm's ability "to create successful alliances, based on learning about alliance management and leveraging alliance knowledge inside the company" (Draulans et al., 2003, p. 152) does in fact refer to the firm's organisational learning mechanisms that allows the firm to transform its prior alliance experience into accessible lessons on alliance management. Hence, we can conclude that learning and sharing alliance expertise is cru-

\footnotetext{
${ }^{9}$ We take a closer look at (alliance) capability building in section 3.3, examining and discussing the roles of experience and organisational routines in the capability building process ${ }^{10}$ To capture the notion of experience on an empirical level, alliance literature often conceptualises alliance experience in terms of the number of alliances a firm has been involved in over a certain period of time.
} 
cial in the development of alliance capability as they generate the necessary alliance know-how to manage alliances.

\subsubsection{Deriving a definition of alliance capability}

Reviewing the various definitions of alliance capability, we noted several similarities and differences. Alliance capability studies have in common the essential ideas, that:

(a) Firms can develop an organizational capability that is responsible for the effective management of alliances.

(b) Alliance capability is as an organisation-wide proficiency in managing alliances as opposed to alliance expertise residing in individuals inside the firm.

(c) Firms that have developed alliance capability are characterised by having an institutionalised approach to learning about alliance management that facilitates experience and expertise sharing throughout the organisation

Yet, alliance scholars seem to disagree on whether to define alliance capability in terms of its constituent elements or its underlying learning processes. As a result, we find no clear-cut definition of alliance capability in alliance literature. Moreover, we noted that extant definitions often suffer from tautologies and equalisations. Therefore, to alleviate these shortcomings we intend to derive a definition of alliance capability that addresses the multidimensionality of the alliance capability construct.

Combining the insights on the underlying learning processes and the tasks of alliance capability, we can derive that firms are in need of learning mechanisms in order to generate the necessary alliance management knowhow, which in turn is necessary as to address the important managerial issues of alliance formation, operation and evaluation.

Therefore, synthesising these insights, we define alliance capability as the institutionalised approach to learning about alliance management in order to support the organisation in the formation, operation and evaluation of its alliances.

To complement our understanding of the nature of alliance capability, the following section examines how firms develop the capability to manage alliances. 


\subsection{How to develop alliance capability?}

The aim of this section is to describe how firms can develop alliance capability. To this end, we present the theoretical underpinnings of alliance capability research. In particular, we show how scholars have drawn upon evolutionary economics, organizational learning, knowledge-based theory and dynamic capability view of the firm to investigate the organisational determinants of alliance capability building. How firms learn about alliance management is addressed by knowledge based view (Nonaka and Takeuchi, 1995; Grant 1996) and organizational learning theory (Levitt and March, 1988, Nonaka 1994). How firms translate their experience and what they learn from it into repeatable patterns of action, i.e. routines, has been investigated by evolutionary economists (Nelson and Winter, 1982) and dynamic capability scholars (Helfat and Peteraf, 2003; Zollo and Winter 2002; Eisenhardt and Martin, 2000; Teece et al., 1997). The following review intends to illustrate how alliance research has drawn upon these four theories to advance our understanding of alliance capability building. We find these four theories particularly insightful as they demonstrate how the development of capabilities in general and alliance capability in particular rests upon unique sources of knowledge.

\subsubsection{Theoretical underpinnings of organisational capability development}

As previously described in Chapter 2, organizational capabilities, whether dynamic or operational, are conceptualised as bundles of routines; meaning a collection of 'patterned behaviour' that are learned (Nelson and Winter, 1982). The notion of routines was first introduced by Cyert and March (1963) and is now closely associated with the work by evolutionary theorists Nelson and Winter (1982). Seminal contributions to this debate have also been provided by Herbert Simon, regarding the notion of bounded rationality, and Michael Polanyi, regarding the distinction between explicit and tacit knowledge that have lead to the evolutionary perspective of the firm as a 'knowledge processor'. Polanyi (1966) defined tacit knowledge as nonverbalizable, intuitive and unarticulated ("we know more than we can tell" 1966, p.4) and explicit or codified knowledge as transmittable in formal and systematic language and hence storable. Adding a tacit dimension to the traditional concept of knowledge, knowledge was no longer seen as a non- 
exclusive public commodity (Coase 1937) but gained a 'quasi-private' dimension exclusive of the firm. Herewith, evolutionary economists stoutly rejected the neo-classical assumptions of rational agents and profit maximising firms but instead emphasised the centrality of knowledge in production. Nelson and Winter (1982) raised Polanyi's distinction of tacit/codified knowledge from the individual to the organizational (firm) level and posited that tacit knowledge is found in individual skills on the personal level and in routines at the organizational level. Routines present 'rules' that aid the members of organizations to cope with bounded rationality and knowledge processing. As such, routines act as repositories of knowledge (the cognitive aspects of learning) but also act as mechanisms of governance ${ }^{11}$. With the emphasis on tacit knowledge, routines are suggested to function as durable stores for non-codified knowledge, i.e. for procedural memory of the organization (Cohen and Bacdayan 1994).

Regarding the question how routines, and in effect, capabilities are developed, evolutionary economists suggest that routines are the result of past experiential learning efforts and therefore evolve slowly on the basis of performance feedbacks (Nelson and Winter, 1982). Performance feedbacks are gained by experiential wisdom, as they are the results of "trial and error learning and the selection and retention of past behaviour" (Gaveti and Levinthal, 2000:113). Therefore, following the view of evolutionary economists, routines are the building blocks of organizational capabilities and can only be accumulated by experiential learning.

Drawing upon the knowledge based view (Nonaka and Takeuchi, 1995; Grant 1996), scholars emphasise the necessity to transform tacit knowledge generated by experiential learning into codifiable lessons that can be shared in order to enable organization-wide learning and capability building (Zollo and Winter, 2002; Eisenhardt and Martin, 2002, Zander and Kogut, 1995). For instance, as Eisenhardt and Martin (2002, p. 1114) remark, "while repeated practice (experience accumulation) per se can contribute to the evolution of capabilities, the codification of that experience into technology and formal procedures makes that experience easier to apply and accelerates the

\footnotetext{
${ }^{11}$ Cohen et al. (1996) suspect that the conceptual difficulty on the exact definition of routines in literature stems from this double character of routines ascribed by Nelson and Winter (1982).
} 
building of routines". Nonaka (1994) explicitly documents the knowledge conversion process and interaction between tacit and explicit knowledge. He distinguishes four types of knowledge-related processes that form a 'spiralling' knowledge process interaction between explicit and tacit knowledge. 'Socialisation' involves the sharing of tacit knowledge through shared experience while 'combination' "involves the use of social processes to combine different bodies of explicit knowledge held by the individual" (p.19). These first two types of Nonaka's knowledge-related processes do not imply any transformation of tacit into explicit knowledge, yet the following two do: The third mode of knowledge conversion, 'externalisation', converts tacit knowledge into explicit knowledge, while the fourth mode 'internalisation' transforms explicit knowledge into tacit knowledge.

Building upon this concept, Zollo and Winter (2002) propose two learning mechanisms, knowledge articulation and codification, that convert tacit into explicit knowledge and hence support the development of routines. Knowledge articulation describes the expression of internalised (thus tacit) lessons learnt, opinions and beliefs. It is a widely shared opinion within the scholarly debate that knowledge articulation allows important collective learning to take place (Nonaka and Takeuchi, 1995, Argyris and Schon 1978, Duncan and Weiss 1979). Through collective discussions, debriefing sessions and performance evaluations, members of the organization can gain a clearer understanding of the overall performance implications of their actions and learn more effective ways to execute certain organizational tasks (Zollo and Winter, 2002). Cowan, David and Foray (2000) observe that firms differ considerably by the degree to which they transform potentially articulable knowledge into actually articulated statements.

Knowledge codification reaches beyond knowledge articulation. This term describes how individuals codify their understanding of outcome implications of their actions in the form of written tools such as guidelines or project management software that is intended to support the execution of future tasks (Zollo and Winter, 2002). Through the codification process, causal linkages between actions and outcome implications are made explicit, which allows for improved coordination and implementation of complex activities. Hence, knowledge codification facilitates the diffusion of tacit knowledge after it has been articulated (Winter 1987, Zander and Kogut 1995, Nonaka 1994). 
Consequently, drawing on these theoretical insights, Zollo and Winter (2002) emphasise that organisational capabilities "emerge from the coevolution of tacit experience accumulation processes with explicit knowledge articulation and codification activities" (p.344). Similarly, Kale et al (2002, p.749) emphasise that "organizational capabilities develop as a result of recombining and/or integrating knowledge within the firm" and that "firms will likely be more effective at capability development when they develop mechanisms that are purposefully designed to accumulate, store, integrate, and diffuse relevant organizational knowledge acquired through individual and organizational experience". ${ }^{12}$

\subsubsection{Building alliance capability}

Based on these theoretical perspectives on organisational capability building, alliance scholars have suggested various processes, routines, and mechanisms for building alliance capability. In the course of reviewing these studies, the reader will soon note that these terms are used loosely and interchangeably in alliance literature. Often we find additional terminologies such as 'management tools and techniques'(e.g. Parise and Casher, 2003, Duysters and De Man, 2002) which refer to the underlying processes of alliance capability building. Since all these studies draw on the same theoretical foundation, we can safely assume that these different terms commonly refer to a repeatable and standardised pattern of action with regard to alliance management. Hence, we will use routines and mechanisms interchangeably as well. Interesting for our review are the different types of mechanisms

\footnotetext{
12 As can be seen from this quote (Zollo and Winter, 2002) it must be noted that Zollo's and Winter's usage of their key terms is anything but clear-cut. Due to these ambiguities, it remains unclear whether they refer to experience or knowledge. Another important shortcoming is that Zollo and Winter (2002), Winter (1987), Zander and Kogut (1995), Nonaka (1994) and Nonaka and Takeuchi (1995) claim, on the one hand, that tacit knowledge can not be formalised but, on the other hand, they seem to suggest that it can be articulated and thus made explicit. Here lies a fundamental contradiction of all approaches that operate directly or indirectly on the basis of Nonaka's and Takeuchi's concept of the knowledge spiral: that tacit knowledge cannot be written down but obviously can be articulated in words that can be written down. Unfortunately, this obvious contradiction has not been made subject of the ongoing debate so far and therefore has not been resolved yet. Thus, it must be stated that these approaches, however influential and meaningful they are for the subject under scrutiny, are built upon a proposition that represents rather an improvable axiom than validated (academic) knowledge.
} 
described in alliance literature that are suggested to underlie alliance capability building.

One group of alliance studies focuses on alliance learning mechanisms. Indirectly referring to the Nonaka 's (1994) SECI model ${ }^{13}$ and building upon Zollo and Winter's (2002) notion of knowledge articulation and codification, Kale and Singh (2007) propose an 'alliance learning process' that entails the articulation, codification, sharing and internalization of alliance management know-how. "Such learning processes may be especially important in the context of building skills to manage tasks or activities that occur repetitively" (Kale and Singh, 2007, p. 984) and hence support the development of alliance capability. For instance, the authors suggest the use of debriefing sessions and record keeping of all major alliance-related activities to facilitate knowledge articulation, the use of collective meetings to encourage individual managers to share their personal alliance management experience, and the use of in-house and external alliance trainings to foster knowledge internalisation.

An important step in these learning processes is the codification of alliance know-how associated with prior experience into accessible lesson on alliance management. These can take the form of guidelines, manuals and standardised processes to support and guide support alliance staff in the execution of their daily tasks. Firms can use standardised processes with regard to partner selection (Harbison and Pekar, 1998) alliance evaluation and monitoring benchmarking (Draulans et al., 2003) and facilitating (or protecting, if needed) knowledge exchanges between partner firms. Individually tailored partner programmes, partner portals, intranet, joint business planning and the use of mediators encourage joint problem solving and allow for mutual value creation.

Other studies emphasise the importance of establishing an alliance infrastructure consisting of alliance staff, communication and incentive systems that support the day-to-day execution of the firm's alliance strategy (Bamford et al., 2003). To allocate resources and coordinate alliance relevant activities, a number of alliance management mechanisms need to be designed

\footnotetext{
${ }^{13}$ SECI presents the four knowledge conversion modes of socialization, externalization, combination, and internalization
} 
that specifically act as a central coordination mechanisms (Harbison and Pekar, 1998) or coordinative capacity (Teece and Pisano, 1994; Kale and Singh, 1999; Nault and Tyagi, 2001). Establishing an alliance department (Kale et al., 2002) or alliance office (Borker et al., 2004) facilitates the coordination of alliance-related activities. Here, alliance scholars emphasise the importance of individual positions or units dedicated to the management of alliances (Spekman et al, 1999) To further support the execution of alliance management, sophisticated incentive systems (Greenhalgh, 2001) are part of the alliance infrastructure and induce continuity among personnel, a crucial condition for a continuous base of knowledge absorption in the alliance (Leonard-Barton, 1995). Rewards and bonuses schemes can prove a useful means to this end (Aldrich, 1999).

Duysters and de Man (2002) collecting up to thirty-four alliance management mechanisms from various sources in academic and managerial publications provide one of the most comprehensive overviews of different alliance mechanisms. Table 1 gives an overview showing these mechanisms divided into four categories. The first group of alliance mechanisms are termed 'alliance tools', which are practical mechanisms that aid the process of day-to-day management of alliances. The second group of alliance mechanisms consist of alliance functions, which refer to individual positions or units dedicated to the management of alliances. The third group of alliance mechanisms is found in control and management processes that ease coordination between individuals and units by establishing both tacit and explicit rules for coordination (Kogut and Zander, 1996). External parties, such as consultants, lawyers, financial experts or conflict mediators form the fourth group of alliance mechanism. External parties can complement the alliance knowledge of the company and bring in a good portion of objectivity and alliance experience. 
Table 3.2: $\quad$ Alliance management mechanisms

\begin{abstract}
Alliance Tools
Alliance best practices, alliance database, alliance handbook, alliance management development program, alliance metrics, competency framework, cross-alliance evaluation, culture programme, external alliance training, in-house alliance training, individual evaluation, intranet, joint business planning, partner portal, partner programme, standard partner selection approach
\end{abstract}

\title{
Alliance Function
}

Alliance department, alliance managers, alliance specialist, gatekeeper, vice-president of alliances

\begin{abstract}
Alliance Processes
Formal experience exchange, approval processes in place, rewards and bonuses for alliance manager
\end{abstract}

\section{External parties}

Consultants, financial experts, legal experts, mediators for conflict resolution

Source: Duysters and de Man (2002)

Clearly, alliance literature is filled with examples of alliance mechanisms and routines. Part of these studies focus on a single or a few alliance mechanisms only. Due to the exclusive focus on a particular type of alliance mechanism, these studies do not indicate (1) whether different types of alliance mechanisms are necessary to build alliance capability and if so, (2) what different types of alliance capability underlie the development of alliance capability. For instance, the study by Kale et al (2007) rightly emphasises the importance of mechanisms for knowledge articulation and internalisation - yet without specifying whether these types of mechanisms suffice for alliance capability building or whether other mechanisms are necessary as well. Since each study focuses on a certain type of alliance mechanism, no comprehensive understanding is generated.

Other studies, such as by Duysters and de Man (2002) provide an exhaustive list of various alliance mechanisms that are at the disposal of companies to support alliance management. However, it does not become clear how these mechanisms contribute to the development of alliance capability. The question arises, what are the underlying purposes of these mechanisms in alliance capability building.

Therefore, in the following section we intend to derive different types of alliance mechanisms that are necessary for alliance capability building. We 
classify types of alliance mechanisms in terms of the purpose they serve in alliance capability building.

\subsubsection{Discussion}

Reflecting on the various examples of alliance mechanisms, we find that two issues are recurring in many studies. While some studies emphasise learning mechanisms, other studies focus on mechanisms that support the execution of alliance management. Therefore, we find that alliance capability mechanisms serve two important purposes in capability building:

(a) knowledge augmentation and transfer,

(b) knowledge application.

In the first case, mechanisms are designed to augment and transfer alliance know-how associated with prior experience. Examples of these mechanisms are collective meetings to encourage sharing of personal alliance management experience and the use of in-house and external alliance trainings to foster knowledge internalisation (Kale and Singh, 2007). In the second case, mechanisms are designed to apply alliance know-how to the management of alliances. Here, we can differentiate between mechanisms that constitute an alliance infrastructure responsible for the coordination and execution of alliance related activities (e.g. alliance office, alliance staff), and standardised processes that guide alliance staff in the execution of alliance related activities (e.g. standardised partner selection programs, alliance evaluation techniques).

Therefore, we delineate three types of alliance mechanisms according to their purpose in alliance capability building:

1) knowledge augmentation and transfer mechanisms

2) alliance infrastructure and functions

3) standardised management processes

By designing these different types of mechanisms, firms can develop alliance capability, which in turn supports them in the management of their alliances (i.e. supports the firm in the formation, operation and evaluation phase of their alliances.)

Clearly, these three types of alliance mechanisms are closely intertwined. An alliance department (Kale et al. 2002) or alliance office (Borker et al.,2004) provides the infrastructure in which alliance staff carries out its daily tasks 
of alliance management with the help of guidelines and standardised processes. However, it is also responsible for the accumulation and internalisation of alliance know-how by means of knowledge augmentation and transfer mechanisms, and the codification of this know-how into standardised management processes and guidelines. Hence, an alliance infrastructure facilitates the augmentation and transfer of knowledge within the organisation as well as the execution of alliance know-how to the management of the firm's management

Table 3.3 indicates the three categories of alliance mechanisms and provides examples of mechanisms for each of these categories. Hereby we adopt the list of alliance mechanisms provided by Duysters and de Man (2002) as this list provides the most comprehensive overview in extant alliance capability literature. Noteworthy, the categorisation by Duysters and de Man (2002) does not reflect the classification of mechanisms into three categories. Rather, their first category 'alliance tools' appears to comprise both 'learning mechanisms' (e.g. training programmes) as well as standardised processes (alliance evaluation). To rearrange these thirty-four alliance mechanisms gathered by Duysters and de Man (2002) into our three categories of alliance mechanisms, we examine each of these mechanisms separately and classify them according to the main purpose they serve in alliance capability building.

For our first category 'knowledge augmenting and transfer mechanisms', we include all training programmes and mechanisms that facilitate the sharing of experience residing in individuals (intranet, in-house knowledge, best practices). For our second category 'infrastructure and functions', we include alliance department, alliance staff, incentive schemes, as well as internal and external experts as these introduce the firms to novel alliance management practices and guide the firm's management in alliance-relevant legal and financial matters. For our third category 'standardised processes,' we include various alliance evaluation techniques and metrics, as well as mechanisms that facilitate the interaction and communication with alliance partners (e.g. joint business planning, partner programs, partner portals). 
Table 3.3: Alliance mechanisms categorised in terms of main purpose in alliance capability building

\begin{tabular}{l|l|l}
\hline $\begin{array}{l}\text { Knowledge augmentation and } \\
\text { transfer mechanisms }\end{array}$ & $\begin{array}{l}\text { Infrastructure and } \\
\text { functions }\end{array}$ & Standardised processes \\
\hline In-house alliance training & Alliance department & Individual evaluation \\
\hline External alliance training & Alliance managers & Joint evaluation \\
\hline Culture program & Alliance specialist & Cross alliance evaluation \\
\hline Intercultural training & Vice president & Alliance metrics \\
\hline In-house knowledge & Local alliance managers & Alliance handbook \\
\hline Formal experience exchange & Gatekeeper & Standard partner selection \\
\hline $\begin{array}{l}\text { Alliance management develop- } \\
\text { ment program }\end{array}$ & $\begin{array}{l}\text { Rewards for alliance } \\
\text { managers }\end{array}$ & Approval processes \\
\hline Best practices & $\begin{array}{l}\text { Rewards for business } \\
\text { managers }\end{array}$ & Alliance database \\
\hline Competency framework & Mediators & Partner program \\
\hline Intranet & Financial experts & Partner portal \\
\hline & Legal experts & Joint business planning \\
\hline Total: 10 & Consultants & Country specific policies \\
\hline
\end{tabular}

\subsection{Conclusion}

Our examination of the notion of alliance capability revealed two important issues in extant literature. First, we found no single clear-cut definition of alliance capability. While one type of definitions indicates the underlying learning processes that enable firms to build alliance management expertise, other definitions address tasks of alliance management. However, we argue that a definition of alliance capability needs to specify both the underlying organisational determinants as well as the tasks of alliance capability. Discussing literature on these subjects, we define alliance capability as the institutionalised approach to learning about alliance management in order to support the organisation in the formation, operation and evaluation of its alliances.

We then reviewed alliance literature on alliance capability building and noted a distinct emphasis on the important role of organisational routines and mechanisms in capability building. Alliance literature has generated numerous examples of alliance routines and mechanisms that are important 
for alliance capability building. However, alliance studies usually emphasise one type of alliance mechanism. As a result, alliance literature remains fragmented and lacks a comprehensive understanding of the different types of alliance mechanisms needed to build alliance capability.

To address this shortcoming, we reviewed various examples of alliance mechanisms and categorised these with regard to their main purpose in alliance capability building. Synergising the insights on what constitutes alliance capability and how firms develop alliance capability, we draw following conclusion. As we have discussed, the formation, operation and evaluation of alliances are the three important tasks of alliance management.

To excel in these tasks and to attain successful alliance outcomes, the firm is in need of developing alliance capability by

(1) designing routines to acquire, integrate and diffuse alliance knowhow,

(2) creating an alliance infrastructure and functions (including alliance office and staff) to coordinate and apply alliance know-how to the tasks of alliance management,

(3) generating standardised processes that guide alliance staff in the management of the firm's alliances.

These routines and processes facilitate an institutionalised approach to learning about alliance management. Therefore, we can refine our definition of alliance capability by incorporating our insights on the different types of alliance mechanisms. Consequently, we restate our definition of alliance capability as the firm's specific learning routines, infrastructure and standardised processes that support the firm in the formation, operation and evaluation of its alliance relationships. 


\section{CHAPTER 4 DATA AND SAMPLE}

\subsection{Introduction}

The main goal of this thesis is to investigate how firms can successfully manage their portfolio of alliances. By adopting an alliance capability view to researching this question, we are particularly interested in the effectiveness of alliance capability mechanisms in raising alliance portfolio performance. In the preceding theoretical chapters, we discussed the important roles and types of alliance mechanisms in building alliance capability. In the succeeding empirical chapters, we intend to examine empirically the effectiveness of alliance mechanisms in raising alliance portfolios. To this end, we will take into account the heterogeneity in firms' levels of alliance experience and alliance portfolio configurations and thus examine the effectiveness of alliance mechanisms given a certain level of experience and portfolio configuration. By taking into account that firms differ in their level of alliance experience and portfolio configuration, we can give recommendations that are more precise with regard to which alliance mechanisms are most effective in raising portfolio performance.

In order to research how firms can successfully manage their portfolio of alliances, we need data on the

(1) performance rates of firms' alliance portfolios

(2) usage of alliance capability mechanisms by firms

(3) level of alliance experience accumulated by firms

(4) size of firms' alliance portfolios

(5) diversity in firms' alliance portfolios

Our review of existing, publicly accessible databases indicated that none of these provided comprehensive information on our five issues. For instance, the 'Securities Data Company (SDC) Platinum' database from Thomson Financial, or the CATI-MERIT database has been used by numerous alliance researchers to collect data on strategic alliances (Anand and Khanna, 2000; Bae and Gargiulo, 2004; Kale et al., 2002; Reuer et al., 2002). However, these databases provide no data on the individual firms' management practices, 
levels of alliance experience or portfolio configurations. Therefore, it became apparent that we could not rely on secondary data, but needed to create an own, proprietary, dataset.

In the following sections, we describe the motivation and background of this study (section 4.2), explain the research design (section 4.3), describe the design of the questionnaire (section 4.4) and the execution of the survey (section 4.5). Finally, we provide a description of our sample (section 4.6). Please note that the descriptions of our variables and analytical techniques for our empirical analyses will be provided separately in the respective chapters.

\subsection{Motivation and Background}

Given the strong coincidence of academic research interests and business orientation of this study, it was desirable to design the empirical part of this thesis in a way that, on the one hand, guarantees that relevant firms (i.e. firms that active in alliances), are inquired and, on the other hand, that the research questions cover topics that are of interest to practitioners and academia.

To this end, we have established a close collaboration with the Association of Strategic Alliance Professionals (ASAP), a non-profit organization established in 1998 with 2000 members worldwide. ASAP's main objectives include the dissemination of research on alliance best practices, the provision of alliance educational programs and repository, and exchange of alliance management relevant knowledge among its members. Consequently, this organisation provides the probably best partner that can be found for a study with the objectives described above.

Since 2002, ASAP supports regularly surveys on its members in order to deepen insights in the functioning and problems of alliances and alliance management. Our study constitutes the 'second state of alliance management'. Before the survey used for this study was carried out, the first survey took place in 2002. While the overall topic of these surveys remained the same (i.e. to assess the state of alliance management among firms globally), the concrete questions that were asked differed from survey to survey. In particular, our survey included additional questions on the size and structure of alliance portfolios in order to allow us to investigate how firms can manage their alliance portfolios most successfully. 


\subsection{Research design}

In the following, we describe the main choices we faced when designing the research for this study. The purpose of this study is mainly descriptive, as we aim to investigate how firms can successfully manage their alliance portfolios by means of accumulating alliance experience and the use of alliance mechanisms. We chose for a statistical study versus a case study, as we aim for breadth rather than depth. A statistical study allows us to test our hypotheses and research questions quantitatively.

The time dimension of our study is set to be cross-sectional as to allow us to take a snapshot of one point in time. A longitudinal study seemed desirable as well, as to allow us to track changes in alliance management and performance over time. However, given the limited time and budget available for this study, we chose a cross-sectional setting.

Regarding the method of data collection, we had to consider whether to conduct our survey by means of personal or telephone interviews or by means of mail or on-line survey. We choose for an online questionnaire as to reach as many companies as possible with little costs involved.

ASAP provided us with their contact list of companies that are active in alliances; that is, all respondents indicated to be involved in at least one alliance relationship. The contact list included 6612 email addresses. Therefore, we defined our target population as these 6612 contacts of alliance managers and VP of companies. We decided to contact each of these persons. Our sample would include all persons that answered our questionnaire. 


\subsection{Designing the questionnaire}

The questionnaire is divided into four parts. In total, we posed 59 questions in total. ${ }^{14}$

(1) Company demographics

(2) Alliance background

(3) Types of alliances formed

(4) Alliance capability mechanisms

The first section of our questionnaire inquired the demographics of the responding firms. Respondents were asked to indicate the 'number of employees of parent company', the 'total worldwide sales volume of the previous year' and 'the primary industry' in which the company is active. To ease the answering process, we provided 'ranges' for the first two questions, and a list of industry names as well as the option to write the industry down.

The second section of our questionnaire investigated the alliance background of the respondents. In particular, we asked about the performance rate of the firm's alliances as well as the firm's alliance experience. One important point of discussion during the development of this questionnaire regarded the appropriate measurement of alliance performance. Essentially, alliance literature on measuring alliance performance is divided into two categories. While some scholars use objective, financial measures such as revenues and costs (e.g. Contractor and Lorange, 1988) or profitability and sales growth (Mohr and Spekman, 1994), others advocate the subjective managerial assessment of alliance performance (e.g. Anderson and Narus, 1992; Beamish, 1984; Hebert and Beamish, 1997; Mohr and Spekman, 1994; Parkhe, 1993; Saxton, 1997). With the exception for joint ventures that are separate legal entities, we find that traditional financial or accounting measures are incapable of capturing whether or not the alliance has been a success in terms of fulfilling its initial goals. Therefore, in line with previous studies, we relied upon the managerial assessments of alliance performance,

\footnotetext{
14 Noteworthy, as we collaborated with ASAP and Cisco Company, we included eight additional questions that were not directly relevant to this study.
} 
measuring alliance success as the percentage of alliances where the firm's initial goals were realized (Kale, Dyer and Singh, 2002).

Important to note, the level of analysis of our study is the performance of the entire alliance portfolio. Traditionally, alliance studies focussed on the performance of the firm's individual alliance. Since we are interested to examine how firms can successfully raise the performance of their alliance portfolio, the later is the unit of analysis for this study. For the respondents of our questionnaire this implied that they had to indicate the company's overall alliance success rate; i.e. the percentage of alliances where the initial goals were realized over the last five years.

Furthermore, to capture the notion of experience on an empirical level, alliance literature often conceptualises alliance experience in terms of the number of alliances a firm has been involved in over a certain period of time (Anand and Khanna, 2000, Duysters and de Man, 2002). Following this approach, we asked respondents 'how many alliances the company has established over the last five years'.

The third section of the questionnaire inquired about the types of alliance formed by the companies. These questions were relevant as to gain information on the composition of firms' alliance portfolios. We inquired about the size of firm's alliance portfolios as the number of simultaneous operational alliances (Duysters and de Man, 2002, 2007; Heimeriks et al. 2008). We further inquired about the types of functional activities in companies' alliance portfolios (e.g. R\&D alliances, marketing alliances, etc), as well as the share of foreign alliance partners, inter-industry alliance partners and competitors in companies' alliance portfolios.

The fourth section of the questionnaire inquired the usage of alliance capability mechanisms. Based on the exhaustive list by Duysters and De Man (2002), we investigate the use of thirty-four different alliance mechanisms (please refer to table 3.2 in Chapter 3). The investigation of these key variables of alliance capability are represented by thirty-four single-item dummy variables, hence, the existence of a certain mechanism is measured by a nominal variable. Therefore, a binominal scale (end-points of the scale consist of two bipolar activities, i.e. yes versus no) is used to understand if a company has a certain mechanism in place $\left(\mathrm{X}_{\mathrm{C}}=1\right)$ or $\operatorname{not}\left(\mathrm{X}_{\mathrm{C}}=0\right)$. 


\subsection{Execution of survey}

We collected a sample of corporate data on firm's alliance portfolios, alliance management mechanisms, and overall alliance performance rates. An online survey questionnaire was developed in cooperation with the Association of Strategic Alliance Professionals to cover these areas of interest. The membership database of this association was used to address the mailings to the key informants on firms' alliance activities and related management practices, in this case to the vice presidents or top managers in charge of corporate alliance management. These persons were used as key informants on their firm's alliance activities and related management practices. Each person received an invitation by email with a personalized link to the online questionnaire, stating the purpose and importance of the research, as well as assuring the confidentiality of the response. The online survey was accessible for 3 months (November 2006 until January 2007). A follow-up letter was sent two weeks after the initial mailing in order to optimise the response rate. A total of 181 responses from alliance managers were collected $^{15}$; of these, 97 responses were collected before the reminder was sent and the remaining 84 responses were accumulated before the end of January 2007. Excluding the emails that were returned because the address was apparently wrong, the response rate is about 3\% (6612 "valid" contacts in the database, 181 respondents). Although we did not get a high response rate in general, we still collected a sufficiently large sample to perform meaningful statistical analyse

\section{Non-response analysis}

Samples may suffer from unit non-response bias, i.e. when a potential respondent cannot be reached or refuses to answer. To ensure that our dataset is not biased due to unit non-response, the data is screened to compare a number of respondent characteristics. These characteristics are examined to understand if the sample is a good representation of the population. An analysis is performed to verify if any unit non-response bias is apparent using three variables to compare early versus late respondents. According to

\footnotetext{
15 The 181 responses entail responses by companies and their business units. We treat these business units as economically independent subunits and therefore weight their answers as equally important as responses given by the parent company.
} 
Kanuk and Berenson (1975) as well as Armstrong and Overt (1977) late respondents can be assumed to be comparable to non-respondents. Respondents were categorized as early respondents if they completed the online questionnaire before the send out of the follow up mail (16.Nov. - 27.Nov 2006) and late respondents completed the survey after the follow up mail (28. Nov. - 24.Jan. 2007). These three variables are firm size measured by the number of employees of the parent company as well total worldwide sales revenue and alliance performance. Chi-square tests for each of these measures show there is no difference between the two categories of respondents. None of the three variables was significant at the $5 \%$ level. The chi-square statistics show that no significant correlations were found between item scores and survey response time. This indicates that there is no significant unit non-response bias in our dataset.

To ensure that our dataset is not biased due to item non-response (i.e. respondents do not answer all the questions) we used the Expectationmaximization (EM) method to detect nonrandom pattern of missing values. The EM Correlation matrix (graph too large to include) tests the hypothesis of a random pattern of missing values. The Little's MCAR test generates following test results: Chi-Square $=2077.167, \mathrm{DF}=2144$, Sig. $=.846$. The hypothesis of random pattern of missing values cannot be rejected. Consequently, this indicates that there is no significant item-non-response bias in our dataset.

\subsection{Description of sample}

Table 4.1 depicts the distribution of firm size and sectoral breakdown of the companies in our sample. The majority of companies $(67 \%)$ in our sample are large, with an employee base greater than one thousand persons. Next, companies with less than 500 employees are well represented (26\%) as well. Only a minority of $5 \%$ of the companies have an employee base between 500 and 1000 employees. The respondents are from a variety of sectors; while the majority of firms (21\%) operate in the information and technology sector (ICT), 13\% operate in pharmaceutical/biotechechnology industries while $12 \%$ of companies are found in the software sector. 
Table 4.1: $\quad$ Sample description

\begin{tabular}{l|l}
\hline & Percentage \\
\hline Firm size (number of employees) & \\
\hline small (<500) & $28,2 \%$ \\
\hline medium (500-1000) & $5 \%$ \\
\hline large (>1000) & $66,9 \%$ \\
\hline Primary industry & \\
\hline ICT & $21 \%$ \\
\hline pharma/biotech & $13 \%$ \\
\hline software & $12 \%$ \\
\hline manufacturing & $8 \%$ \\
\hline financial services & $7 \%$ \\
\hline public sector & $7 \%$ \\
\hline chemicals & $2 \%$ \\
\hline
\end{tabular}

This sectoral breakdown is in line with previous findings that especially in key industries such as telecommunication, computer hard- and software, pharmaceuticals and biotechnology players frequently engage in strategic alliances to access new and valuable resources, share the risks and rewards for R\&D intensive activities and maintain entire alliance portfolios (Wassmer, 2008). The majority of the respondents (68\%) originated from the USA; 25\% belonged to the European Union. Our sample includes a number of major 'global players', often associated with telecommunications and computer hard-and software industries.

Regarding the types of alliances used by responding companies, most alliances in our sample (48\%) are market oriented, meaning that they involve co-marketing, co-promotion or business development alliances. Next, $14 \%$ of alliances in our sample are research alliances. Distribution, supplier and coproduction alliances are less frequent (table 4.2). 
Table 4.2: $\quad$ Types of alliances used by companies

\begin{tabular}{l|l}
\hline Type of alliances & Frequency (\%) \\
\hline Co-marketing alliances & $48 \%$ \\
\hline Research alliances & $14 \%$ \\
\hline Distribution alliances & $13 \%$ \\
\hline Supplier alliances & $11 \%$ \\
\hline Co-production alliances & $10 \%$ \\
\hline Other & $4 \%$ \\
\hline
\end{tabular}

Regarding the distribution of alliance portfolio performance, table 4.3 illustrates that, a small group of companies (9\%) are highly successful with alliances. In fact, more than $80 \%$ of their alliances are a success. On the other hand, there is also a small group of companies $(9 \%)$ that have extremely low success rates that are less than $20 \%$. This distribution is in line with previous findings (Duysters and de Man, 2002, 2007).

Table 4.3: Alliance performance distribution

\begin{tabular}{l|l}
\hline Alliance success rate & $\begin{array}{l}\text { Frequency (\%) } \\
\text { percentage }\end{array}$ \\
\hline $0-20 \%$ & $9 \%$ \\
\hline $21-40 \%$ & $27 \%$ \\
\hline $41-60 \%$ & $27 \%$ \\
\hline $60-80 \%$ & $28 \%$ \\
\hline $81-100 \%$ & $9 \%$ \\
\hline Total & $100 \%$ \\
\hline
\end{tabular}

Table 4.4 shows an overview of alliance mechanisms and their frequencies, which make up the explanatory variables of this study. As the table illustrates, some alliance mechanisms are used more often than other mechanisms. For instance, alliance managers, the implementation of in-house knowledge, joint business planning and individual evaluation are the most frequently used alliance mechanisms in our dataset. Alliance mechanisms such as legal experts, intercultural training and mediators are the least frequently implement mechanisms by companies. 
Table 4.4: Frequency of implemented alliance mechanisms

\begin{tabular}{|c|c|}
\hline Alliance mechanism & Frequency \\
\hline Alliance managers & $84 \%$ \\
\hline In-house knowledge & $82 \%$ \\
\hline Joint business planning & $82 \%$ \\
\hline Individual evaluation & 81 \\
\hline Partner program & $74 \%$ \\
\hline Joint evaluation & $74 \%$ \\
\hline Approval processes & $72 \%$ \\
\hline Local alliance managers & $70 \%$ \\
\hline Best practices & $70 \%$ \\
\hline Intranet & $60 \%$ \\
\hline Alliance metrics & $69 \%$ \\
\hline Alliance department & $67 \%$ \\
\hline Standard partner selection program & $66 \%$ \\
\hline Partner portal & $62 \%$ \\
\hline Rewards for alliance managers & $60 \%$ \\
\hline Alliance specialist & $58 \%$ \\
\hline Alliance database & $56 \%$ \\
\hline VP of alliances & $52 \%$ \\
\hline Cross-alliance evaluation & $50 \%$ \\
\hline Financial experts & $50 \%$ \\
\hline Formal exchange of experience & $48 \%$ \\
\hline In-house alliance training & $47 \%$ \\
\hline Country specific policies & $43 \%$ \\
\hline Rewards for business managers & $43 \%$ \\
\hline External alliance training & $42 \%$ \\
\hline Consultants & $41 \%$ \\
\hline Competency framework & $41 \%$ \\
\hline Alliance handbook & $37 \%$ \\
\hline Alliance management development program & $35 \%$ \\
\hline Gatekeeper & $30 \%$ \\
\hline Cultural program & $28 \%$ \\
\hline Mediators & $24 \%$ \\
\hline Intercultural training & $21 \%$ \\
\hline Legal experts & $12 \%$ \\
\hline
\end{tabular}

Based upon the presented characteristics, we can reasonably assume that our sample is representative for the most active firms engaging in strategic alliances. A detailed description of our variables and analytical techniques are provided in the respective empirical chapters. 


\subsection{Conclusion}

It is the main goal of this study to assess how firms can successfully manage their portfolio of alliances. By adopting an alliance capability view, we focus on alliance mechanisms as a main determinant of alliance portfolio performance. Accordingly, we have designed a survey, which investigates the usage of thirty-four alliance mechanisms, the performance rates of firm's alliance portfolios, the level of firms' alliance experiences and the configuration of firms' alliance portfolios. This particular survey design allows us to assess the effectiveness of alliance mechanisms in raising the performance of firms' alliance portfolios given a firms' level of alliance experience and type of alliance portfolio configuration.

Based on our data sample, we are going to perform a number of statistical tests in the succeeding chapters. In Chapter 5, we assess the effectiveness of alliance mechanisms in raising alliance performance given a firm's specific level of alliance experience. Hereby we are going to identify how firms can most effectively capitalise on their existing experience base by means of specific alliance mechanisms. In Chapter 6 and 7, we identify which alliance mechanisms are most effective in raising performance given the particular configuration of a firm's alliance portfolio. Specifically, we identify which alliance mechanisms are most effective in addressing the particular challenges that accompany an expansion in alliance portfolio size (Chapter 6) and which alliance mechanisms are best suited to address the challenges that arise with different levels of alliance portfolio diversity (Chapter 7).

Consequently, based on the results of these three empirical chapters we are able to generate a comprehensive overview of alliance mechanisms that are most effective in raising alliance portfolio performance given a firm's level of experience and type of alliance portfolio configuration. 



\section{Chapter 5 Developing Alliance Capability}

\subsection{Introduction}

One of the fascinating aspects of researching alliances is the considerable heterogeneity in alliance performance rates among firms. While some succeed, the majority of firms struggle to turn their alliances into a success (Duysters and de Man, 2002, 2007). This heterogeneity in alliance performance rates has triggered scholars and practitioners alike to reveal the critical success factors of alliance performance. In Chapter 2, we discussed important theoretical perspectives on alliance success factors. We saw that traditional alliance literature has polarised on relational (trust, cultural fit) and structural factors (choice of governance, resource fit) to explain conditions for alliance success, while the emerging alliance capability view has directed the focus to the internal, organisational determinants of alliance performance. Adopting the latter perspective, Chapter 3 discussed theories on capability building. Essentially, theory suggests two important organisational determinants of alliance capability building, namely the accumulation of alliance experience and the use of alliance mechanisms. In practice, the question arises how firms can build alliance capability in order to raise performance levels.

In the current chapter, we aim to evaluate empirical alliance capability literature with regard to the extent to which this question has been explored, and what issues remain to be answered. Overall, we can identify three distinct groups of empirical alliance capability research. (To proxy the roles of alliance experience and alliance mechanisms in alliance capability building, extant empirical studies commonly test for a significant association to firmlevel alliance performance).

The first group of studies exclusively examines the direct effect of alliance experience on alliance performance. While some studies confirm a positive relationship between alliance experience and performance (e.g. Shan et al., 1994; Anand and Khanna, 2000), other scholars maintain that accumulating alliance experience does not increase alliance success indefinitely (e.g. Rothaermel and Deeds, 2006). 
The second group of studies investigates the relative contribution of alliance mechanisms to alliance performance, indicating that some alliance mechanisms are more effective than others at raising alliance performance (e.g. Heimeriks 2005; Kale et al. 2002).

The third group of studies suggests that at different experience levels different alliance mechanisms are most effective in enhancing firm-level alliance performance (Draulans, et al. 2003; Heimeriks, Duysters and Vanhaverbeke, 2007).

Evaluating state-of-the-art empirical alliance capability research, we identify the following crucial shortcomings: By either examining alliance experience or alliance mechanisms as sole determinants of alliance performance, the first and second group of studies seem to investigate two sides of the same coin. The third group of studies, however, does account for the important roles of both alliance experience and mechanisms in alliance capability building and further differentiates the effectiveness of alliance mechanisms as a function of a firm's level of alliance experience.

Given the nascent stage of alliance capability research, the relationship between alliance experience, alliance mechanisms, and alliance performance remains ambiguous. In order to respond to these shortcomings, this chapter is dedicated to researching the following three questions: (a) what is the relationship between alliance experience and alliance performance, (b) which alliance mechanisms are most effective in raising alliance performance and (c) is the effectiveness of alliance mechanisms dependent on the firm's level of alliance experience?

In addressing these issues, we aim to extend current empirical work and hereby to contribute to the theoretical development of the alliance capability discipline.

To answer our questions, we will first review and discuss state-of-the-art empirical research on the relationships between alliance experience, alliance mechanisms and firm-level alliance performance. By means of our quantitative survey of 175 companies, we assess how firms can effectively develop alliance capability and raise alliance performance rates. 


\subsection{The roles of alliance experience and alliance mechanisms in raising alliance performance}

\subsubsection{Alliance experience, alliance mechanisms and firm-level alliance performance}

One stream of alliance research has investigated whether the accumulation of alliance experience raises alliance performance. ${ }^{16}$ Several studies analysed the direct effect of alliance experience on alliance performance (Shan et al., 1994; Anand and Khanna, 2000; Kale et al., 2002). Shan et al. (1994) find a strictly positive relationship between alliance experience and alliance outcome where prior alliance experience spurs innovativeness of high-tech start-ups. Along the same lines, investigating more than 2000 joint ventures and licensing agreements, Anand and Khanna (2000) find that experienced partner firms in $R \& D$ joint ventures created more value as their inexperienced counterparts.

While acknowledging the important role of alliance experience, other scholars emphasise the importance of learning mechanisms for alliance capability building. As Kale et al. (2002) remarks, alliance experience is a necessary but not sufficient condition for alliance capability building as alliance experience may have little impact unless the tacit experience is internalised into accessible lessons on alliance management (Kale et al., 2002). Rather, by the use of learning mechanisms, firms are able to capture, share and disseminate alliance management know-how, associated with prior experience.

The limitations to the beneficiary role of alliance experience for superior alliance performance is best illustrated by Draulans et al (2003) who find that accumulating alliance experience does not increase alliance success indefinitely. Draulans et al. (2003) find a curvilinear relationship between the number of alliances a firm establishes (which they conceptualise as a proxy for measuring alliance experience) and the performance rate of these alliance portfolios (illustrated in figure 5.1). As Figure 5.1 indicates, the success of an organisation's alliances rises as it enters into more alliances. However, a limit to learning-by-doing is reached (at the point of six alliances) where little improvement in alliance success is gained from entering into further alliances (Draulans et al., 2003: 155).

16 Clearly, measures for alliance experience and alliance performance differ among these studies. Chapter 4 discusses different approaches on how to measure these variables. 
Figure 5.1: Relationship between the number of alliances (proxy for alliance experience) and the success of the organisation's alliances.

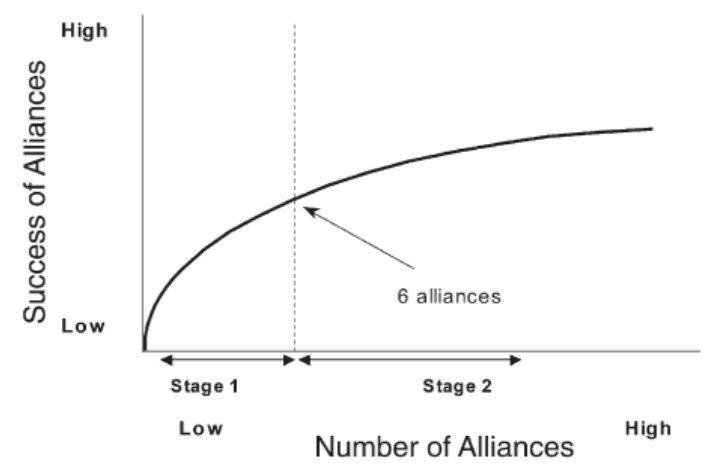

Source: Draulans et al. (2003)

Therefore, a majority of alliance capability studies has shifted its focus to investigating the role of alliance mechanisms in raising firm-level alliance performance.

Broadly speaking, alliance capability studies usually adopt one of three common methods to test the relationship between alliance mechanisms and alliance performance. One method is focussed on the exclusive testing of a single alliance mechanism. Usually, these studies first present the selected alliance mechanisms in great detail and then test for its impact on firms' alliance performance. Studies by Kale et al. (2002) and Borker et al. (2004) prove illustrative of this method. Measuring firm-level alliance success through abnormal stock market gains following alliance announcements and managerial assessments of long-term performance, Kale et al. (2002) propose that firms that created a 'dedicated alliance function' (i.e. a separate organizational unit dedicated to the management of alliances) achieve greater alliance success. Herewith, Kale et al. (2002) implicitly assume that the use of a dedicated alliance function can be seen as a proxy to the presence of a firm's alliance capability. Moreover, Borker et al. (2004) similarly focus on one particular alliance mechanism, advocating the implementation of a socalled 'alliance office' that ensures the coordination of all alliance-related activities and thus is suggested to secure firm-level alliance performance. An alliance office is defined to be responsible for 'institutionalizing supportive processes and tools, developing and sharing alliance know-how, embedding the right alliance mindset and analysing alliance patterns in order to learn 
from experience' (Borker et al., 2004:4). Especially the notion of a 'dedicated alliance function' has been quickly adopted among alliance capability scholars. Important to notice, many scholars seem to be content to limit their examination to only this alliance mechanism. For instance, Lichtenthaler and Lichtenthaler (2004) recommend the use of an alliance function to manage international R\&D alliances, without considering other potentially important alliance mechanisms. Similarly, Sarkar et al. (2009) exclusively examine the importance of a dedicated alliance function in managing alliance portfolios.

A second method operationalises alliance capability as the sum of a firm's alliance management practices. For instance, in the study by Heimeriks and Duysters (2007) alliance capability is proxied by the sum of a firm's alliance management practices, ranging from a score between zero to thirty-four, depending on the number of mechanisms in use (Heimeriks and Duysters, 2007:35). Their study confirms a positive relationship between alliance capability (sum of mechanisms) and alliance performance.

Yet, as a third group of studies illustrates, firms can choose from a variety of alliance mechanisms to manage their alliance. In this regard, Duysters and de Man (2002) have conducted one of the most comprehensive studies on this subject, identifying and collecting up to thirty-four alliance management mechanisms that present a proxy for the alliance related organizational processes..$^{17}$ This groundbreaking list has been (fully or partially) adopted by subsequent studies, which probe the importance of these mechanisms for alliance capability building and alliance performance (Draulans et al., 2003; De Man, 2005; Heimeriks, 2005; Heimeriks, Duysters and Vanhaverbeke, 2007; Heimeriks and Duysters, 2007).

For instance, an interesting application of this list of thirty-four alliance mechanisms is conducted by De Man's (2005) comparison of the strength of alliance capability in Europe and North America, which reveals significant differences in the usage of these mechanisms between these two regions.

\footnotetext{
${ }^{17}$ Since this is the most comprehensive list and because this differentiation is widely accepted in alliance management research, it has also been used for the ASAP survey (2007) that provided the data to this study (please refer to Chapter 4). This provides an opportunity to associate and compare results across different studies and contribute new insights in this part of the academic and practical discourse.
} 
European firms were found to rely heavily on know-how of individual employees and lack institutionalised processes for managing alliances.

In contrast to their American counterparts, only a few European firms were found to have built up professional alliance management.

Moreover, the relative contribution of these mechanisms on alliance performance is tested by a study of Heimeriks (2005). By means of discriminant analysis, his results indicate that high-performing alliance firms have a significant higher number of important functions in place than low-performing firms, e.g. the use of VPs of alliances, alliance departments and alliance managers are found to significantly enhance alliance performance.

Evaluating different empirical methods that are commonly used to examine the relationship between alliance mechanisms and firm-level performance, we find two main points of criticism. Our first critique is aimed at studies that exclusively test a single alliance mechanism. On the one hand, these studies provide us with a detailed picture of a single alliance mechanism e.g. describing the merits of an alliance department (e.g. Kale et al., 2002; Borker et al., 2004). This is indeed necessary as to gain a better understanding of the process of alliance management. On the other hand, this exclusive focus on a single alliance mechanism implicitly suggests that the usage of one alliance mechanism is a proxy for the existence of a firm's alliance capability. Such an approach does not adequately capture the theoretical understanding that organizational capabilities exist of various routines and mechanisms that are implemented simultaneously to achieve an end result in the capability development process (Helfat and Peteraf 2003; Zollo and Winter 2002; Nelson and Winter, 1982.; Prahalad and Hamel, 1990). ${ }^{18}$ Empirically speaking, the exclusive focus on a single alliance mechanism neglects the fact that, in practice, firms use an array of different alliance mechanisms in order to build alliance capability.

Our second critique is targeted at studies that operationalise alliance capability as the sum of a firm's alliance mechanisms (e.g. Heimeriks and Duysters, 2007). While this method of viewing a firm's alliance capability as the aggregate of all its mechanisms may be appropriate in the context of the study by

\footnotetext{
${ }^{18}$ Please refer to Chapter 3 for a detailed review and discussion of theories on alliance capability building
} 
Heimeriks and Duysters (2007), this approach implies the assumption that all mechanisms equally contribute to raising alliance performance.

In contrast, the third group of studies has illustrated that a dedicated alliance function does not have to be the sole alliance mechanism of interest, and that different alliance mechanisms exhibit different contributions to firm-level alliance performance.

Given the scant empirical evidence on the important roles of alliance mechanisms in alliance capability building, we intend to extent current research by Heimeriks (2005) by examining:

Question 1: Which alliance mechanisms are most effective in raising firm level alliance performance rates?

\subsubsection{Levels of alliance capability building}

Synthesising the insights on alliance experience and alliance management mechanisms as determinants of alliance capability, research suggests that at different experience levels, different alliance mechanisms are most effective in enhancing firm-level alliance performance (Draulans et al., 2003; Heimeriks, Duysters and Vanhaverbeke, 2007).

Building upon the important insight that inexperienced companies learn differently about alliance management than experienced companies, Draulans et al (2003) propose an 'ideal learning trajectory' for developing alliance capability where alliance learning mechanisms should be adjusted to each level of experience. To enhance learning, Draulans et al. (2003:161) suggest that inexperienced firms should enter into a number of similar alliances and learn by means of alliance training courses as well as individual alliance evaluation techniques. Experienced firms, on the other hand, profit from the use of cross- alliance evaluation techniques and the use of alliance specialists.

Along the same line, Heimeriks, Duysters and Vanhaverbeke (2007) find that at different stages of alliance capability building, different learning mechanisms have different performance effects. Figure 5.2 illustrates the alliance capability development model, which largely combines insights from Crossan et al (1999), Draulans et al. (2003) and Helfat and Peteraf (2003). Each of the three capability curves illustrated in Figure 5.2 represents a different 
stage of alliance capability development related to a particular alliance experience level.

Figure 5.2: Levels of alliance capability development.

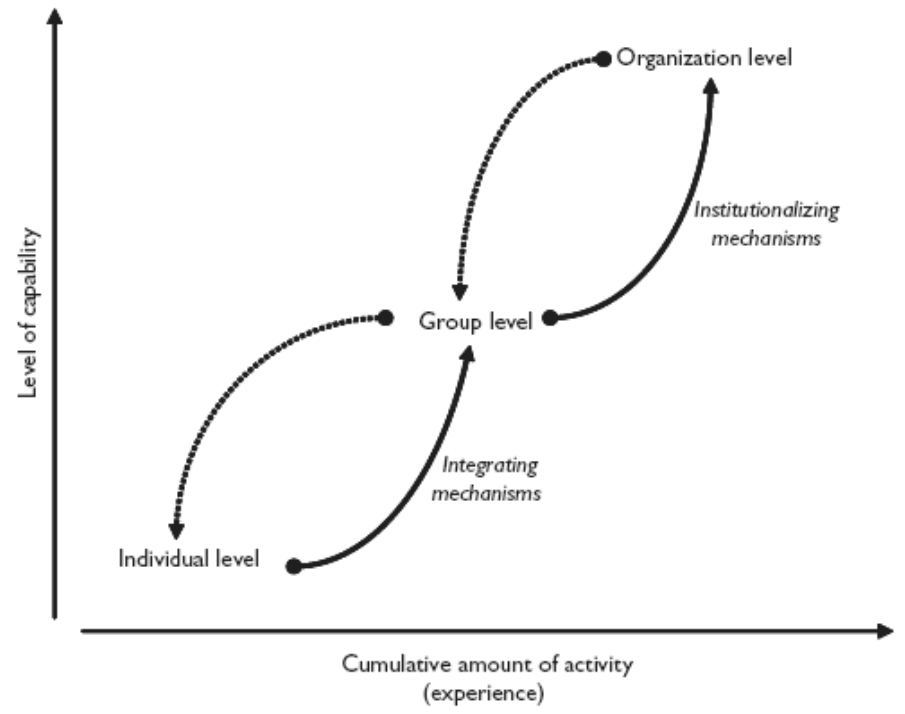

Source: Heimeriks et al. (2007, p.377)

While the first development stage is associated with 'individual learning', the second development stage involves 'group level learning' and the third stage is linked to 'organizational level learning'. To evolve from one development stage to the other, Heimeriks et al. (2007) propose two types of learning mechanisms that facilitate the transfer of experience. Integrating mechanisms are suggested to enable the exchange of individual experiences and are therefore associated with group-level learning.

Examples of integrating mechanisms include internal alliance training, external alliance training, training in country differences, alliance best practices, culture programme, comparisons of alliance evaluations and alliance metrics. Institutionalising mechanisms are suggested to foster the routinisation and formalisation of practices inside the firm and are therefore associated with organisational-level learning (Heimeriks et al., 2007:377).

Examples of institutionalising mechanisms include alliance department, vice-president of alliances, alliance managers, local alliance managers, part- 
ner selection programme, intranet, rewards for alliance managers tied to alliance performance, formally structured exchange between alliance managers and country-specific alliance policies. Empirically, the authors provide the important insight that experienced alliance firms make relatively more use of institutionalising mechanisms (i.e. mechanisms to foster routinisation of practices inside the firm), while inexperienced firms make relatively more use of integrating mechanisms (i.e. mechanisms to enable the exchange of individual experiences) (Heimeriks, Duysters Vanhaverbeke, 2007).

To conclude, these two studies give us reasonable grounds to expect that the effectiveness of alliance mechanisms depends on the firms' level of alliance experience. Following the logic of these studies, we like to investigate:

Question 2: Which alliance mechanisms are most effective given the level of alliance experience?

By addressing this question, we intend to refine the current empirical research method in an important way. Notably, Draulans et al (2003) investigate the performance implications of three selected alliance mechanisms (evaluation methods, alliance specialist, alliance training). However, solely examining three alliance mechanisms does not generate a full picture on the variety of alliance mechanisms that can be effective at different levels of alliance experience.

Furthermore, Heimeriks et al. (2007) distinguish two groups of alliance mechanisms (integrating and institutionalising mechanisms) that are each represented by the aggregate of all separate items (i.e. seven items in the case of integrating mechanisms and nine items in the case of institutionalising mechanisms). Hereby, Heimeriks et al. (2007) test the overall importance of these two groups, but not the effectiveness of the underlying individual alliance mechanisms.

In an attempt to extend and refine research by Heimeriks et al. (2007) and Draulans et al. (2003), we like to include all thirty-four alliance mechanisms and examine the effectiveness of each of these alliance mechanisms separately at different levels of alliance experience. Hereby, we intend to generate comprehensive empirical evidence on the effectiveness of alliance mechanisms at different levels of alliance experience. 


\subsection{Method: variables and analytical techniques}

In the course of this chapter, we have derived a number of questions on the effect and interrelationship between alliance experience and alliance mechanisms in raising firm- level alliance performance. In the remaining of this chapter, we intend to examine these questions empirically. Given the nascent stage of alliance capability research, a large empirical study allows the rigorously testing of our questions and is expected to generate critical theoretical as well as practical insights into effective alliance capability building. To this end, our survey included a number of questions on this matter; e.g. inquiring respondents about their companies' level of alliance experience, alliance performance level, and use of alliance mechanisms. The details on our survey and sample are provided in Chapter 4 'Methodology'; the corresponding questionnaire is included in Appendix 1. In the following, we provide a brief overview of the variables and analytical techniques used to investigate our research questions.

\subsubsection{Dependent and independent variables}

Our aim is to investigate how firms can increase firm-level alliance performance; hence, alliance performance is our dependent variable. To measure alliance performance, we relied upon the managerial assessments of firmlevel alliance performance, measuring alliance success as the percentage of alliances where the firm's initial goals were realized (e.g. Kale, Dyer and Singh, 2002). Our original variable on alliance performance has five categories, with a distribution as indicated in Chapter 4, figure 4.3. For the purpose of this chapter, we are interested to examine how high performing firms differ from lower performing firms in the use of alliance mechanisms. Therefore, we created a dichotomous dummy variable, which defines high performing firms to have a success rate of $61-100 \%$, while other firms score 0 $60 \%$.

We measured alliance experience, one of the independent variables, as the number of previously established alliances over the period 2001-2006 (Duysters and De Man, 2007). Our original variable on alliance experience has five categories with a distribution as indicated in table 5.1 
Table 5.1: Distribution of alliance experience

\begin{tabular}{l|l|l}
\hline Alliance experience & Frequency & Valid percentage \\
\hline $1-5$ alliances & 61 & $35 \%$ \\
\hline $6-15$ alliances & 70 & $40 \%$ \\
\hline $16-25$ alliances & 26 & $15 \%$ \\
\hline $26-40$ alliances & 4 & $2 \%$ \\
\hline $40+$ alliances & 12 & $7 \%$ \\
\hline Total & 173 & 100 \\
\hline
\end{tabular}

In order to generate meaningful analyses by means of logistic regressions and chi-square testing, we needed to create a new variable with only three categories. These three categories are defined as

- low level of alliance experience (1-5 alliances)

- moderate level of alliance experience (6-12 alliances)

- high level of alliance experience (16 and more alliances)

The distribution of this variable is reasonable with $35 \%$ of companies having a low level; $40 \%$ of companies having a moderate level, and $25 \%$ of companies having a high level of alliance experience.

In order to investigate how firms manage alliance portfolios, our survey asked companies to indicate whether they used any of the thirty-four alliance mechanisms depicted in table 3.3 (Chapter 3). Each of these mechanisms are measured as dichotomous dummy variables (yes versus no) and represent independent variables. Therefore, companies can score up to a maximum of thirty-four alliance mechanisms in total. Hence, our survey provides us information on which alliance mechanisms are used by companies as well as the overall number of alliance mechanisms adopted by companies.

\subsubsection{Analytical techniques}

Firstly, to test whether different alliance mechanisms exhibit different marginal contributions to firms' alliance performance, we use chi-square based statistics (cross-tabulations) to examine which alliance mechanisms are positively associated to a high alliance performance rate. The reason why we choose chi-square statistics rather than regression analysis is that a preliminary testing of our dataset revealed that our thirty-four alliance mechanisms are correlated to each other, that is, the use of one alliance mechanisms is 
associated with the use of other alliance mechanisms. To test all thirty-four alliance mechanisms simultaneously in the same regression model would distort our results, as the multicollinearity does not allow us to differentiate the effects of one alliance mechanism from the other.

Secondly, to test whether at different experience stages, different alliance mechanisms exhibit different marginal contributions to firm-level alliance performance, we again rely on chi-square-based statistics as the appropriate statistical measure for the association between alliance mechanisms and alliance performance. In particular, we differentiate three levels of alliance experience, and within each level, we further differentiate between low and high performing firms. We then test within each level of experience which mechanisms are associated with high performing firms.

\subsection{Analysis and results}

The first step in our analysis is to assess which alliance mechanisms are most effective in raising alliance performance. Performing cross-tabulations between alliance mechanisms and firm-level alliance performance shows that some alliance mechanisms contribute more significantly to alliance performance than others. Table 5.2 lists seventeen out of the overall thirty-four alliance mechanisms that are found to significantly influence a firm's alliance performance rate, that is, each of these seventeen mechanisms is positively associated with high performing firms (i.e. having a success rate of $60-100 \%$ ). The first six alliance mechanisms (from top of left column) exhibit highly significant associations (at $\mathrm{p}<0.01$ ), while the remaining variables are moderately significant for increasing alliance performance. Alliance mechanisms that are not listed in this table have no significant impact on alliance performance outcome. 
Table 5.2: $\quad$ Critical success factors

\begin{tabular}{|c|c|}
\hline \multicolumn{2}{|c|}{ Most effective alliance capability mechanisms to raise alliance performance } \\
\hline Alliance management development program *** & Alliance managers ${ }^{* *}$ \\
\hline Cross alliance evaluation ${ }^{* * *}$ & Financial experts** \\
\hline Individual evaluation ${ }^{* * *}$ & Culture program ${ }^{* *}$ \\
\hline Alliance metrics $* * *$ & Alliance specialist* \\
\hline Joint evaluation ${ }^{* * *}$ & Alliance database * \\
\hline Consultants & Partner portal ${ }^{*}$ \\
\hline Competency framework ${ }^{* *}$ & Legal experts* \\
\hline Intercultural training ${ }^{* *}$ & Approval* \\
\hline In-house knowledge ${ }^{* *}$ & \\
\hline
\end{tabular}

${ }^{*} \mathrm{p}<0.10, \quad{ }^{* *} \mathrm{p}<0.05, \quad{ }^{* * *} \mathrm{p}<0.01$

Based on the results of table 5.2, we can conclude that not all mechanisms are equally useful to raise alliance performance, but that some mechanisms are indeed more effective in raising performance rates than other mechanisms are.

Secondly, we wonder if all of our seventeen critical success factors are useful to all firms, or whether firms with different levels of alliance experience are in need of different alliance mechanisms to raise performance rates. Therefore, we examine whether for different levels of alliance experience, different alliance mechanisms will prove effective in raising alliance performance. To test this, we will first examine if firms with different levels of alliance experience differ significantly in the usage of alliance mechanisms. Next, we inspect each level of alliance experience separately, testing which alliance mechanisms lead to higher alliance performance at different levels of alliance experience.

We use one-sided ANOVA to investigate whether the usage of alliance mechanisms differs significantly among the three categories of alliance experience. The results are shown in table 5.3. As the F-value is not significant, we must conclude that firms with different levels of alliance experience do not differ significantly regarding the overall number of alliance mechanisms adopted. 
Table 5.3: Average adoption by absolute number of alliance mechanisms per alliance experience level (ANOVA)

\begin{tabular}{l|l|l|l|l}
\hline & $\begin{array}{l}\text { low level of } \\
\text { experience }\end{array}$ & $\begin{array}{l}\text { moderate } \\
\text { experience }\end{array}$ & $\begin{array}{l}\text { high level of } \\
\text { experience }\end{array}$ & F-value \\
\hline $\begin{array}{l}\text { number of mechanisms } \\
\text { adopted }\end{array}$ & $15,79(7,53)$ & $17,44(7,11)$ & $17,61(6,16)$ & 1,185 \\
\hline $\mathrm{N}$ & 61 & 70 & 42 & \\
\hline
\end{tabular}

There are 34 alliance mechanisms in total; $\mathrm{N}=169$;

Cells provide means (in absolute numbers) and standard deviation for the number of alliance mechanisms adopted. ${ }^{*} \mathrm{p}<0.10 ;{ }^{* *} \mathrm{p}<0.05 ;{ }^{* * *} \mathrm{p}<0.01$

Next, we run chi-square tests to examine whether and how firms with different levels of alliance experience differ in the usage of alliance mechanisms. The results of our cross-tabulation test indicates, that surprisingly, firms with different levels of alliance experience do not differ significantly the usage of alliance mechanisms. So far, we must conclude that firms with different levels of alliance experience use not only the same number of mechanisms in total, but also do not differ significantly in the usage of alliance mechanisms.

Lastly, we examine the effectiveness of each alliance mechanism at different levels of alliance experience. An alliance mechanism that is effective at raising performance for a firm with a low level of experience can be less effective at higher levels of alliance experience.

Table 5.4 summarises our results of the cross-tabulations, where we have examined the effectiveness of alliance mechanisms for each level of alliance experience. In particular, we find that in the category of low alliance experience level, high performing firms make significantly more use of individual evaluations and financial experts than low performing firms do.

In the category of moderate alliance experience level, high performing firms use significantly more often alliance metrics, joint evaluation, alliance database, individual evaluation, partner portals, in-house alliance training, intranet, alliance management development program, consultants, alliance managers, vice presidents and rewards for alliance managers as compared to low performing firms. In the category of high alliance experience level, high performing firms use significantly more often alliance metrics, cross-alliance evaluation, culture programs and in-house knowledge as compared to their low performing counterparts. 
Table 5.4: Successful mechanisms given level of alliance experience

\begin{tabular}{|c|c|}
\hline $\begin{array}{l}\text { Level of alliance experi- } \\
\text { ence }\end{array}$ & $\begin{array}{l}\text { Significant alliance mechanisms } \\
\text { (associated with high performing firms) }\end{array}$ \\
\hline Low experience level & individual evaluation* , financial experts* \\
\hline $\begin{array}{l}\text { Moderate experience } \\
\text { level }\end{array}$ & 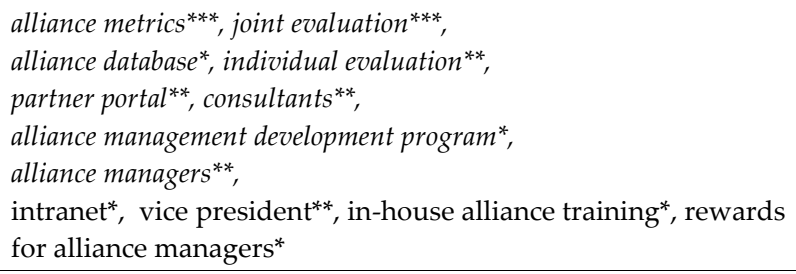 \\
\hline High experience level & $\begin{array}{l}\text { alliance metrics*, cross-alliance evaluation } \\
\text { cultural program }^{*}, \text { in-house knowledge }\end{array}$ \\
\hline
\end{tabular}

${ }^{*} \mathrm{p}<0.10,{ }^{* *} \mathrm{p}<0.05,{ }^{* * *} \mathrm{p}<0.01$

By examining which mechanisms are used by high performing as compared to low performing firms we can confirm our expectation that for different levels of alliance experience, different mechanisms are effective in raising performance. Notably, the usage of a critical alliance mechanism by high performing firms is not exclusively limited to one level of alliance experience. For instance, the use of alliance metrics is found to increase alliance performance for firms with moderate as well as with a high level of alliance experience.

Interesting to note is the comparison between our seventeen critical success factors that raise alliance performance in general (table 5.2) and our results for significant alliance mechanisms at given levels of alliance experience (table 5.4). We make the following observations:

Surprisingly, not all of our seventeen general success factors (table 5.2) can be found in table 5.4. Only eleven out of the seventeen general success factors (table 5.2) are present in either one or more categories of alliance experience (table 5.4). Hence, these eleven success factors are now associated with a certain level of alliance experience. We have marked these mechanisms in italics in table 5.4.

Six out of our seventeen general success factors (table 5.2) are not part of our list in table 5.4. These six general success factors are indeed 'general' in the sense that they are not associated with a certain level of alliance experience but seem to raise alliance performance regardless of the level of alliance ex- 
perience. These six success mechanisms are: competency framework, intercultural training, alliance specialists, partner portals, legal experts, approval processes.

Moreover, table 5.4 generates four additional alliance mechanisms that were not indicated as critical success factors in table 5.2. These four alliance mechanisms are only significant at a moderate experience level. These four alliance mechanisms are: intranet, vice-presidents, in-house alliance training and rewards for alliance managers.

\subsection{Discussion and conclusion}

In this chapter, we investigated how firms can most effectively raise the performance of their alliances by developing alliance capability. In particularly, we assessed the relationship and interplay between accumulating alliance experience and the use of alliance mechanisms in raising alliance performance. Our empirical analysis has generated three crucial insights:

1) The accumulation of alliance experience does not raise alliance performance

2) While some alliance mechanisms always raise alliance performance (regardless of alliance experience level), other mechanisms only raise performance in conjunction with a particular experience level

3) There is a considerable gap between what firms do versus what is effective

Taken together, our results indicate a different path to alliance capability building than laid out in extant literature. We will discuss each of our three results and derive the theoretical and practical implications of our findings.

First, to build alliance capability, various studies suggested that gaining experience through repetitive engagement in alliances improves managerial practices and eventually spurs alliance performance. The results of our own empirical analysis, however, indicate that there is no significant relationship between accumulating alliance experience and firm-level alliance performance. Hence, our finding diverges from the prominent argument in alliance capability literature. 
One reason for these diverging empirical results on the effects of alliance experience surely lies in the different methodological approaches adopted among empirical studies (e.g. differences in defining and measuring alliance experience). Another reason, however, might be of a more fundamental nature. In the past, accumulating alliance experience might have just been sufficient to boost alliance performance rates. However, nowadays it seems that a vast number of firms acknowledge the importance of building alliance capability and increasingly invest in alliance mechanisms to manage their alliances (e.g. Duysters and De Man, 2007). Hence, accumulating alliance experience might be a necessary but not sufficient condition for raising alliance performance and gaining an advantage vis-à-vis rivals Simonin, 1997; Kale et al. 2002, Eisenhardt and Martin, 2002).

Second, alliance literature suggests various alliance mechanisms to build alliance capability and to raise alliance performance. Our empirical analysis has extended prior work by differentiating which alliance mechanisms are 'always' effective in raising alliance performance versus which alliance mechanisms are effective given a certain level of alliance experience. We find that six alliance mechanisms raise performance levels regardless of the level of alliance experience. Competency framework, intercultural training, alliance specialists and legal experts are all associated with augmenting and transferring alliance know-how throughout the organisation, while partner portal ease the communication and interaction with the alliance partners. In contrast, other alliance mechanisms are only effective given a certain level of alliance experience.

Testing which alliance mechanisms are most effective given the level of alliance experience, we find that the use of individual evaluation techniques and financial experts is sufficient to raise the alliance performance with little prior alliance experience. For companies with a moderate level of alliance experience, the use of numerous knowledge augmenting and transfer mechanisms (e.g. training programmes, intranet), standardised processes (e.g. alliance evaluation techniques, alliance database, partner portals) and alliance functions (e.g. alliance department, alliance managers, vice presidents, consultants) is necessary to raise alliance performance. For companies with a high level of alliance experience, a relatively smaller number of mechanisms is sufficient to manage their alliances successfully, namely, in- 
house knowledge and cultural programmes to disseminate alliance relevant knowledge as well as alliance metrics and cross-alliance evaluations to assess the performance of their alliances.

To a certain degree, our results are in line with studies by Heimeriks et al. (2007) and Draulans et al. (2003). In particular, our result reflects the main message of these studies, namely, that firms with different levels of alliance experience need different alliance mechanisms to enhance performance rates. ${ }^{19}$ Similar to the study by Draulans et al. (2003), our results indicate that firms with a low level of alliance experience benefit most from individual alliance evaluations while firms with a high level of alliance experience rely on cross-alliance evaluations to raise performance rates. In contrast, we find that alliance training programmes are more beneficial to experienced firms that inexperienced ones.

The study by Heimeriks et al. (2007) ${ }^{20}$ indicates that inexperienced firms benefit most from integrating mechanisms (e.g. various training programs and evaluation techniques) while experienced firms benefit mostly from the use of institutionalising mechanisms (e.g. alliance functions). In contrast, we find that firms with moderate alliance experience employ a variety of mechanisms associated with knowledge augmentation, standardised processes and alliance functions to raise performance levels. Firms with a high level of alliance experience, however, solely rely on a few standardised processes to manage their alliances.

Reflecting on our results, the theoretical implications of our findings suggests a different path to alliance capability building. By definition, firms with low alliance experience have been involved in a relatively small number of

19 Methodologically speaking, all three studies (including ours) are similar in the definition of key variables: alliance experience (i.e. number of previously established alliances over a five year period), alliance performance (i.e. extent to which alliances met initial goals) and alliance mechanisms (based on the list provided by Duysters and De Man, 2002).

20 Comparing our results to the study by Heimeriks et al. (2007) is less straightforward. Heimeriks et al. (2007) base their study on the same list of thirty-four alliance mechanisms that we have used in our survey. However, they select sixteen alliance mechanisms for their analysis and run a factor analysis to group these mechanisms into either 'integrating mechanisms' or 'institutionalising mechanisms'. The presence of these two factors is measured by the aggregate of all its separate items (i.e. seven items in the case of integrating mechanisms and nine items in the case of institutionalising mechanisms) and therefore does not provide any further insight regarding the contribution of the individual alliance mechanisms to raising alliance performance at respective levels of alliance experience. 
alliances in the past. In contrast to extant studies, we do not find the use of training programmes to be effective at this early stage of alliance capability building. Rather, the use of individual alliance evaluation techniques, as indicated by our empirical analysis, is most beneficial as it allows the firm to learn from its past mistakes; hence, this 'learning-by-doing' approach seems most appropriate given the low level of prior experience..

Further, we witnessed a considerable gap between what firms with little prior experience do versus what they need. In fact, firms with little prior experience use almost as many alliance mechanisms as firms with higher experience levels, namely sixteen alliance mechanisms, while the use of only two particular alliance mechanisms is sufficient to raise alliance performance. In contrast, firms with moderate level of alliance experience need to employ numerous alliance mechanisms in order to raise alliance performance. Hence, we conclude that certain alliance mechanisms only increase performance when implemented in conjunction with experience.

Interestingly, when the firm has reached a high level of alliance experience, our empirical findings indicate that the use of a few standardised processes is sufficient to raise performance level. Probably, these firms have succeeded in building an effective alliance capability and hence the use of few effective standardised processes to support the day-to-day management makes the difference in alliance performance rates vis-à-vis rivals.

Thirdly, we like to draw the attention to the gap between what firms do versus what is effective. Strikingly, we found that firms with different levels of alliance experience do not differ considerably in the overall number of alliance mechanisms adopted and virtually do not differ with regard to what mechanisms they adopt. A reason for this result might be that, in general, firms tend to use a relatively high number of alliance mechanisms, namely 16 alliance mechanisms on average! One could suppose that some firms use as many alliance mechanisms as possible, without necessarily being aware whether these mechanisms are suitable to their particular level of alliance experience. Therefore, our empirical results of this chapter has provided an important practical insight for firms, namely by indicating which alliance mechanisms are most effective given the level of alliance experience. By taking into account the particular level of alliance experience, the firm can make 
appropriate regarding how to build alliance capability most effectively and eventually raise alliance performance.

Overall, we conclude that in order to build alliance capability and to raise alliance performance, accumulating alliance experience is a necessary but not sufficient condition. Rather our empirical analysis confirms the importance of using alliance mechanisms that augment and transfer knowledge as well as facilitate inter-partner communication and interaction. Concluding we can state, that when building alliance capability the firm needs to pick those alliance mechanisms that are appropriate given its level of alliance experience in order to optimise its performance levels. 


\section{Chapter 6 Developing Alliance Portfolio CAPABILITY}

\subsection{Introduction}

Nowadays, firms increasingly engage in multiple alliances simultaneously, sometimes resulting in portfolios with more than 30 alliances operating at the same time (Lavie, 2007; Duysters and De Man, 2007). It seems that firms can no longer afford to rely on a single high profile alliance relationship to respond to the demands of today's complex business environments. Rather, firms increasingly depend on a number of alliances to share technology and knowledge (Dyer and Nobeoka, 2000; Kale, Singh, Perlmutter, 2000), master strategic uncertainty (Eisenhardt and Schoonhoven, 1996) access foreign markets, and improve competitive positioning vis-à-vis rivals (Gimeno, 2004; Silverman and Baum, 2002). Building a large portfolio of alliances is further associated with positive network effects and the accumulation of social capital (Goerzen, 2007).

The trend towards building large alliance portfolios signifies the strategic importance of alliances for firms. However, success rates of only 50\% (Duysters and De Man, 2007) indicate that while some firms strive, some others fail. Therefore, scholars and practitioners alike are intrigued by the question of how firms can successfully manage alliance portfolios.

Alliance capability literature provides two important indications for answering this question. Firstly, conceptually speaking, scholars refer to the need for developing 'alliance portfolio capability' that addresses the particular managerial challenges that arise in alliance portfolios (Hoffmann, 2005; Sarkar et al., 2009). Herewith, these scholars draw an important distinction between the management of individual alliances versus the management of alliance portfolios. For instance, Hoffmann (2005) differentiates 'multi-alliance management capability' from alliance capability on the single alliance level as the "organizational ability to manage a comprehensive alliance portfolio successfully" (p.123). 
Along the same line, Sarkar et al. (2009) argue for the development of an 'alliance portfolio capability' that specifically addresses the formation, development and integration of alliance portfolios.

Therefore, for answering how to manage alliance portfolios successfully, a differentiation of alliance capability in terms of single vs. portfolio management is in order.

Secondly, empirically speaking, alliance capability scholars have begun to systematically examine alliance mechanisms that underlie the development of alliance portfolio capability; herewith indicating that building alliance portfolio capability requires different alliance mechanisms than building alliance capability on the individual alliance management level (Heimeriks et al., 2008; Duysters, de Man and Wildeman, 1999).

Despite these scholarly efforts, alliance capability research on alliance portfolio management remains relatively scarce. The majority of alliance literature is still influenced by traditionally oriented schools of thought, resulting in a strong focus on either relational or structural factors to explain the conditions for alliance success ${ }^{21}$. In order to develop a more complete alliance capability view on the important issue of alliance portfolio management, we intend to address two crucial shortcomings in extant alliance portfolio literature.

Firstly, the notion of alliance capability on the portfolio level remains unclear. While scholars such as Hoffmann (2005) and Sarkar et al. (2009) emphasise the need for 'alliance portfolio capability', the exact differentiation of alliance capability on the individual versus portfolio management level is scantily discussed. Hence, to advance our understanding on what constitutes alliance portfolio capability, we need to discuss how the management of alliance portfolios reaches beyond the tasks of managing individual alliances.

Secondly, we notice a lack in large-scale empirical studies on how firms can develop alliance portfolio capability. The few existing empirical studies (e.g. Hoffmann, 2005; Heimeriks et al. 2008) provide first indications, yet their contribution is limited to a relatively small number of alliance management

\footnotetext{
${ }^{21}$ In Chapter 2, we have extensively discussed the various schools of thoughts on alliance success factors, contrasting traditionally oriented research streams with the emerging alliance capability perspective.
} 
practices under scrutiny. To respond to this shortcoming, we need to examine a comprehensive set of alliance management practices and to identify which of these are particularly suited to develop alliance portfolio capability.

Therefore, this chapter is dedicated to researching the following two questions: (a) how does the management of alliance portfolios reach beyond the tasks of managing individual alliances and (b) what alliance management practices are most effective at supporting these tasks and hence raise the performance of the alliance portfolio?

In addressing these issues, we aim to alleviate the current shortcomings in alliance (capability) literature and to advance our understanding on what constitutes alliance portfolio capability and how can firms successfully develop alliance portfolio capability. To answer these questions, we will first review and discuss how alliance management on the individual alliance level differs from management at the portfolio level and then derive hypotheses on the alliance management practices that underlie the development of alliance portfolio capability. By means of our quantitative survey among 175 companies, we assess the effectiveness of thirty-four alliance mechanisms for developing alliance portfolio capability and raising alliance portfolio performance rates.

\subsection{Conceptualising alliance capability on individual vs. portfolio level}

Overall, as the preceding chapters have illustrated, we note an overwhelming share of traditionally-oriented alliance studies and only an emerging number of scholars that adopt an 'alliance capability' perspective to investigate individual / portfolio alliance management (please refer to Chapter 2). Because of the nascent stage of alliance capability research, the definition of an alliance portfolio capability is not fully developed. To respond to this shortcoming, we firstly review challenges and managerial tasks that arise on the individual alliance management level and then compare these to alliance portfolio management. Based on our discussion of state-of-the-art literature on this subject, we delineate how the management of alliance portfolios reaches beyond the tasks of managing individual alliances. 


\subsubsection{Alliance management on the individual alliance level}

Without doubt, the management of alliances is a difficult undertaking. Studies that draw upon transaction cost, agency cost and social exchange theory have particularly well documented the various difficulties that arise in alliance relationships ${ }^{22}$. Essentially, any alliance relationship is likely to experience one or more of the following four challenges that can severely jeopardise its successful outcome. As the alliance passes through the stages of its life cycle $^{23}$, some of these challenges become more prominent and need to be addressed:

A first challenge arises due to relational risk. ${ }^{24}$ Given the understanding that the performance of an alliance depends on the effective cooperation between the alliance partners, relational risk refers to the uncertainties associated with cooperative motivation of the alliance partners, where one partner may pursue opportunistic objectives at the expense of the other partners (Parkhe, 1993; Khanna, Gulati and Nohria, 1998; Das and Teng, 2001).

A second challenge arises due to divided authority structures and the physical, cognitive, and cultural distance between partners. The resulting coordination failures can impede the implementation of joint activities, when there are no clear distribution of roles, procedures and responsibilities (Mohr and Spekman, 1994; Park and Ungson; 2001, Luo, 2006).

A third challenge arises due to a lack of information sharing and communication, which exaggerates the adversarial effects of information asymmetries inherent in any alliance relationship. Communication failures can hinder the

\footnotetext{
${ }^{22}$ Chapter 2 provides an extensive account of these theoretical perspectives.

${ }^{23}$ Alliance life cycle generally refers to five phases: (1) development of alliance strategy that sets the goals of the alliance, (2) screening and selection of alliance partners (3) negotiation of the alliance deal, e.g. deciding upon mutual contributions in term of financial or technological investments; (4) daily operations of the alliance, including coordination and distribution of alliance tasks and responsibilities among alliance staff; (5) evaluation of alliance relationship in terms of goal accomplishment. While studies differ on the terminology and number of these life cycle stages, the description of these stages appears quite similar. For further information on alliance life cycles, please refer to Dyer et al (2001), Das and Teng (1997) and Spekman et al. (1996)

${ }^{24}$ In addition, literature identifies 'performance risk' as hindrance to a successful alliance outcome. Performance risk refers to "uncertainty regarding future states of nature" (Ring and Van de Ven, 1992: 92), thus referring to the "probability and consequences that a firm's strategic objectives are not achieved, despite full co-operation" (Das and Teng, 2001:8). Since performance risk is common to all strategic decisions, we rather focus on the implications of relational risk, as the latter is unique to alliance relationships.
} 
effective assessment of uncertainties and value creation opportunities of an alliance relationship (Ring and Van de Ven, 1994; Park and Ungson, 2001).

A fourth challenge arises when personal relations are neglected in the alliance relationship. Such 'underdeveloped' personal relations can prevent the establishment of trust and reciprocity in the alliance, which would be crucial for setting norms and resolving conflicts (Ring and Van de Ven, 1994; Madhok, 1995; Zaheer, McEvily, and Perrone, 1998; Kale, Singh, and Perlmutter, 2000).

Overall, to mitigate relational risks and to reduce the threat of opportunistic behaviour, alliance scholars suggest selecting and choosing appropriate partners (Dacin, Hitt and Levitas, 1997; Geringer, 1991; Hitt et al., 2000); negotiating an appropriate contract (Argyres and Mayer, 2007; Reuer and Arino, 2007); as well as setting a suitable governance structure for the alliance relationship that will lessen any opportunistic intent of the partner firm (Pisano, 1989; Oxley, 1997).

Therefore, based on these various challenges, alliance capability scholars suggest that the management of an individual alliance requires attention to those problems that arise as the alliance relationship passes through the stages of its life cycle. Consequently, alliance capability on the individual alliance level is conceptualised as the firm's ability to form, operate and evaluate alliance relationships on an individual basis (for a review, please refer to Chapter 3).

\subsubsection{Alliance management on the portfolio level}

Alliance literature suggests additional challenges when it comes to the management of an entire of portfolio of alliances as compared to the management of alliances on an individual basis.

In particular, alliance capability scholars argue that the success of an alliance portfolio does not solely depend on the successful outcomes of individual alliances. Rather, the major pitfall that prevents firms from fully realising the value creating potential of their alliance portfolios is to treat an alliance portfolio as the mere collection of individual alliances. Failing to adopt a 'portfolio perspective' results in several management short sights: 
Firstly, a lack of portfolio perspective can result in a suboptimal choice in alliance partners for the alliance portfolio (Sarkar et al. 2009; Duyster et al 1999; Lichtenthaler and Lichtenthaler 2004). At the individual alliance management level, partner selection is about attaining a fit between two parties. At the portfolio level, however, this will not be sufficient. Partner selection has to takes place in the context of portfolio formation, where additional criteria such as assessing redundancy or fit within the portfolio need to be considered. To support such an approach, Sarkar et al. (2009:587) suggest enhancing 'partnering proactiveness' i.e. "an organisation's deliberate efforts to discover and act on new alliance opportunities".

Secondly, Sarkar et al. (2009) highlight that 'systematic relationship imperfections' such as lack of trust and commitment can prevent partners from sharing and combining their resources effectively. Therefore, to reduce the threat of opportunistic behaviour within the alliance portfolio, Sarkar et al. (2009) suggest the use of 'self-enforcing safeguards' that rely on relational aspects rather than on formal, contractual mechanisms.

Thirdly, the firm might fail to leverage knowledge from individual alliances across its portfolio. At the individual alliance management level, knowledge is exchanged between partners. In a portfolio approach, knowledge can be leveraged across partners (Lorenzoni and Baden-Fuller, 1995) and be used to create synergies within the portfolio. To this end, Sarkar et al. (2009) emphasise the importance of portfolio coordination that facilitates the integration and synchronising of knowledge and activities across alliances.

Finally, Bamford and Ernst (2002) note that firms without a portfolio perspective often do not sufficiently monitor and evaluate the development of the alliance portfolio, fail to conduct thorough analysis on the portfolio level and do not examine how well the alliance portfolio is in line with the overall corporate strategy.

On basis of the first three issues, Sarkat et al. (2009) identify portfolio formation, relational governance and portfolio integration as important tasks of alliance portfolio management. Therefore, they define alliance portfolio capability to encompass (1) a proactive dimensions focussed on portfolio formation, (2) a relational dimension relating to the skills in developing pat- 
terns of interaction, and (3) an integration dimension involving crossalliance knowledge transferring processes (p.586).

On the basis of the fourth issue (alliance portfolio analysis and coordination), Hoffmann (2005:124) identifies the following as crucial tasks of alliance portfolio management: (1) to develop a portfolio strategy that sets the main strategic direction of the alliance portfolio such as rules on how and with whom to co-operate; (2) to monitor the portfolio to ensure that the direction and performance of the portfolio is in line with portfolio strategy; (3) to coordinate the portfolio as to attain synergies among alliances in the portfolio while reducing the potential for conflict; and finally (4) to create an alliance management system that provides the infrastructure and tools to support the tasks of alliance (portfolio) management.

Herewith Hoffmann (2005) responds to main concerns voiced by alliance scholars. For instance, Gulati (1998) emphasises the greater need for early coordination among alliances and alliance partners, since the dynamics in one alliance are likely to influence the outcome of others in the portfolio. Further, the prioritisation of alliances (Dyer et al., 2001) and resource allocation (Fricke and Shenhar, 2000) are new managerial factors that need to be taken into consideration. This is in line with other studies that refer to the need for effective coordination mechanisms that optimise the overall result of the alliances (Platje et al 1994; Rickert, 1995).

\subsubsection{Discussion}

Concluding, our brief review has illustrated the importance of adopting an alliance portfolio perspective, which allows the firm to manage its portfolio as a 'coordinated, holistic collective' and is thus likely to create value beyond what can be accomplished if each were managed separately (Sarkar et al., 2009:588). As Fricke and Shenar (2000) argue, managers need to develop a superior perspective that reaches beyond the performance of individual alliances and embraces the aggregate success of alliances. Therefore, alliance portfolio management needs to cover both the management of the individual alliances as well as of the portfolio, thus requiring different flexibilities in dealing with important aspects of both single and portfolio alliance management on issues such as partner selection, evaluation, coordination and knowledge transfer. 
With our question in mind what does constitute alliance portfolio capability, we will briefly discuss the work by Sarkar et al (2009) and Hoffmann (2005) and propose an own definition of alliance portfolio capability. According to Sarkar et al. (2009) alliance portfolio capability encompasses 'partner proactiveness', 'relational governance' and 'portfolio coordination'. According to Hoffmann (2005), alliance portfolio capability encompasses the development of an alliance portfolio strategy, the monitoring and coordination of alliance portfolios and the management system (infrastructure, tools) to support these management tasks.

Reflecting on these suggestions, we come to following conclusions: Firstly, we find that Sarkar's et al. (2009) definition of 'partner proactiveness' can be integrated into Hoffmann's (2005) notion of portfolio strategy development, as partner selection is an important part of portfolio strategy.

Secondly, we agree that relational governance (Sarkar et al., 2009) poses a central task of alliance portfolio management. Instead of solely relying on contractual agreements, the facilitation of communication and interaction between the partners allows for the development of trust and commitment, which in turn enables the partner firms to share and combine resources effectively.

Thirdly, Sarkar' et al. (2009) and Hoffmann' (2005) notion of portfolio coordination appears to be similar. In line with extant literature, portfolio coordination is evidently a crucial task of alliance portfolio management (see for instance Gulati, 1998; Fricke and Shenkar, 2000, Platje et al. 1994; Rickert, 1995).

Fourthly, alliance literature has emphasised the importance of portfolio monitoring to ensure that individual alliances are contributing to the overall goal of the alliance portfolio (e.g. Bamford and Ernst, 2002) and thus we find it to be a major task of alliance portfolio management.

Finally, we do not consider Hoffmann's (2005) creation of 'an alliance management system' per se as a task of alliance portfolio management. Rather, such a management system provides the necessary underlying components (e.g. infrastructure, processes) to enable the firm to develop an alliance portfolio capability in order to manage its portfolio of alliances (see our discussion and definition of organizational determinants of alliance capability development in Chapter 3). 
As a result, alliance portfolio capability needs to address four important managerial issues, which reach beyond the tasks of managing individual alliances. Firstly, when establishing a new alliance relationship, the focal firm has not only to consider the appropriate fit regarding its potential partner, but needs to selects partners that fit the overall goal of the alliance portfolio.

Secondly, the focal firm needs to facilitate the communication and interaction among the partners in the entire alliance portfolio.

Thirdly, the focal firm has not only to enhance the knowledge transfer between itself and its alliance partner, but also to facilitate the knowledge transfer across partners to create synergies within the portfolio.

Fourthly, the focal has not only to monitor and assess the performance of its alliances on an individual basis, but also to evaluate the performance of the portfolio as an entirety.

Table 6.1: What constitutes alliance portfolio capability?

\begin{tabular}{l|l}
\hline $\begin{array}{l}\text { Dimensions of alliance portfolio } \\
\text { management }\end{array}$ & Management tasks \\
\hline portfolio formation & $\begin{array}{l}\text { set alliance portfolio strategy and select partners that fit } \\
\text { the overall goal of the alliance portfolio }\end{array}$ \\
\hline relational governance & facilitate partner communication and interaction \\
\hline portfolio coordination & $\begin{array}{l}\text { prioritisation and knowledge transfer between alliances } \\
\text { within the portfolio }\end{array}$ \\
\hline portfolio evaluation & $\begin{array}{l}\text { monitor and assess the performance of the alliance } \\
\text { portfolio as an entirety }\end{array}$ \\
\hline
\end{tabular}

Having critically discussed and derived dimensions of alliance portfolio capability, the question arises how firms can develop alliance portfolio capability. The aforementioned study by Sarkar et al. (2009) emphasises the need for developing alliance portfolio capability and specifies three dimensions (e.g. partner proactiveness etc.), but decides to maintain a high level of abstraction and deliberately not to create a list of specific routines of each of these dimensions. ${ }^{25}$. The latter, however, is exactly what is needed to investigate

\footnotetext{
${ }^{25}$ Sarkar et al. (2009, p.586): "our decision to restrict ourselves at a higher level of abstraction is driven by both theoretical imperatives of parsimony and operational consistency. Instead of creating a list of specific routines for each of these dimensions, we believe that it is more fruitful and prudent to capture the essence, or fundamental core, of the capability at a higher level of generalization and abstraction".
} 
how firms can develop alliance portfolio capability. Therefore, in the next section we investigate which alliance mechanisms may be most suitable to develop alliance portfolio capability and raise the performance of their alliance portfolios.

\subsection{Developing alliance portfolio capability: the role of alliance mechanisms}

The previous section identified four main areas of alliance portfolio management, namely, alliance portfolio formation, coordination, evaluation and relational governance. As these tasks reach beyond those of managing individual alliances, we can reasonably assume that developing alliance capability on the portfolio level requires different or additional alliance mechanisms than building alliance capability on individual alliance level. Studies by Heimeriks et al. (2008) and Duysters et al. (1999) strengthen our assumption by suggesting a number of alliance mechanisms especially dedicated to the development of alliance portfolio capability.

For instance, in their conceptual work, Duysters et al. (1999) recommend portfolio analysis, partner programmes and alliance databases as effective tools to support the management alliance portfolios. These management tools are suggested to help optimising the alliance portfolio by facilitating moderation and prioritisation. In a portfolio analysis, the firm analyses its existing portfolio and assesses if the portfolio contains all required competences or if new alliances are necessary. Hence, portfolio analysis can serve as a basis for alliance partner search and selection. Conducting portfolio analysis further clarifies the effects of alliances on each other, assessing whether existing alliances are conflicting or synergetic. 'Partner programmes' are suggested to help classify alliance partners in various groups, enabling the firm to keep an overview over a large number of partners. For each group of partners, the focal firm sets rules of engagement that guide the collaborative endeavours with these firms. In an 'alliance database', the focal firm can register its partners and thus keep an overview of its network. Further, the alliance database enables intra-firm knowledge sharing about alliance partners and best practices. In this way, alliance managers can learn about the experiences and best practices of their fellow colleagues, which 
facilitate organizational learning about managing alliance and hence building of alliance capability (Duysters, et al., 1999).

Moreover, in their empirical study, Heimeriks et al. (2008) find that as the firms' focus shifts from the management of individual alliances to alliance portfolios, firms tend to rely increasingly on functional positions, in-house alliance trainings and standardised protocols. Specifically, their study recommends the usage of codified best practices and intercultural training programs to raise the performance rate of alliance portfolios effectively (Heimeriks et al. 2008, p.13).

These two studies give a reasonable ground to assume that the development of alliance portfolio capability requires the usage of alliance mechanisms especially geared to support the tasks of alliance portfolio management. However, in our review of state-of-art literature, we encounter a somewhat indecisive study by Hoffmann (2005). While his study provides a clear differentiation of alliance tasks on alliance portfolio management, his empirical examination of underlying management tools leads to contradictory findings. In particular, his study firstly mentions eighteen 'instruments of alliance management' that are suggested to facilitate the creation, storage, transfer and application of alliance management knowledge ${ }^{26}$. By means of qualitative and quantitative assessments among twenty-five European firms, Hoffmann finds that alliance portfolio management does not necessitate other kinds of management tools than for individual alliance management. According to Hoffmann (p.136), "portfolio management does not require other tools. Proven tools for managing individual alliances need to be adapted to support the specific demands of multi-alliance management" (p.136). Quite surprisingly, he finally asserts that "the tasks of managing alliance portfolios requires specific processes, tools and organisational solutions" (p.140).

In the case of a multi-alliance management situation, Hoffmann recommends a 'centre of competence' to develop and implement a portfolio strategy, portfolio co-ordination and monitoring.

\footnotetext{
${ }^{26}$ By and large, Hoffmann's eighteen 'instruments' resemble a subset of our list of thirty-four alliance mechanisms by Duysters and De Man (2002).
} 
Following the example of Heimeriks et al. (2008) and Duysters et al. (1999) we like to propose which alliance mechanisms are likely to underlie the development of alliance portfolio capability and eventually contribute to the successful performance of the alliance portfolio.

In this regard, Heimeriks et al. (2008) highlight an important issue, namely that the effectiveness of alliance mechanisms to manage alliance portfolios is influenced by the size of the firm's alliance portfolio. The underlying assumption is that with an expansion in alliance portfolio, portfolio relevant tasks become more prominent and hence require more 'advanced' alliance mechanisms (Deeds and Hill, 1996).

For instance, firms that have a small alliance portfolio only need to manage a few alliances and are therefore likely to maintain their focus on the management of individual alliances, while facing little portfolio relevant challenges. In contrast, with an expansion in portfolio size, portfolio relevant issues such as portfolio coordination and monitoring gain importance.

Given the nascent stage of alliance capability research, especially in the field of alliance portfolio management, we find only emerging evidence on how firms can successfully develop alliance capability to manage alliance portfolios. To extend the work by Duyster et al (1999) and Heimeriks et al. (2008), we are interested to investigate which alliance mechanisms are most effective to build alliance portfolio capability given the size of the alliance portfolio.

Drawing on our review of state-of-the art literature, we have the following expectations. Firms with small alliance portfolios focus on the management of individual alliances and therefore do not need to develop alliance portfolio capability, but solely use alliance mechanisms that support the task of individual alliance management. For instance, standardised processes particularly geared towards the management of individual alliances such as individual alliance evaluation techniques, alliance metrics and alliance handbooks may prove effective. Further, given the small number of alliances to handle, the firm is in no need to create an exhaustive alliance infrastructure or appoint alliance functions.

Firms with relatively larger alliance portfolios, however, need to handle not only tasks at the individual alliance management level but also need to pay attention to the additional tasks of alliance portfolio management. Therefore, they are in need to develop alliance portfolio capability in order to handle 
the 'new' tasks of alliance portfolio management. In particular, an increase in alliance portfolio size results in a greater need for portfolio formation, coordination, monitoring and relational governance.

To support the formation of the alliance portfolio, standardized processes such as partner selection programs can help to select partners that fit the overall goal of the portfolio and help to set rules of engagement that guide the collaborative activities with these firms.

To facilitate the operational tasks of the alliance portfolio, creating alliance infrastructure and functions (e.g. alliance managers, specialist) can help to oversee the coordination of the alliance portfolio with special attention to resource allocation and prioritisation (see Dyer et al 2001, Fricke and Shenhar, 2000). In addition, providing alliance management training programs help the firm to develop in-house expertise in managing alliance portfolios. To support the evaluation of the alliance portfolio, standardized processes such as joint evaluations, cross-alliance evaluations, alliance metrics and alliance databases help monitor the development of the alliance portfolio and assess its 'well-being'.

To support relational governance, mechanisms such as joint business planning, partner programmes and partner portals can facilitate the communication and interaction between alliance partners, eventually enhancing interpartner sharing of resources and information.

Hence, to support a large portfolio of alliances, firms can make use of a greater number of alliance functions to help to oversee the coordination of the alliances portfolio and rely on a greater number of standardised processes to guide the monitoring and evaluation of the alliance portfolio. Further, as suggested by Heimeriks et al. (2008) the need for sharing codified lessons (e.g. best practices) rises as ever more alliances are added to the portfolio.

Therefore, we propose the following hypothesis:

$H_{1}$ : with an expansion in alliance portfolio size, firms are likely to increase portfolio success by using mechanisms associated with standardised processes and infrastructure. 


\subsection{Method}

To understand how firms can successfully manage alliance portfolios, our literature review has indicated various alliance mechanisms that can underlie the development of alliance portfolio capability. Next, we intend to examine empirically which alliance mechanisms are most effective in managing alliance portfolios of different sizes. For this purpose, our survey included a number of questions on this matter, e.g. inquiring respondents about their companies' size of alliance portfolio, alliance performance level, and the use of alliance mechanisms. The details of our survey and sample are provided in Chapter 4 'Methodology'; the corresponding questionnaire is included in Appendix 1.

In the following, we provide a brief overview of the variables and analytical techniques used to investigate our research questions.

\subsubsection{Dependent and independent variables}

Our aim is to investigate how firms can increase firm-level alliance portfolio performance; hence, alliance portfolio performance is our dependent variable. To measure alliance performance, we relied upon the managerial assessments of firm-level alliance performance, measuring alliance success as the percentage of alliances where the firm's initial goals were realized (Kale, Dyer and Singh, 2002). Our original variable on alliance portfolio performance has five categories, with a distribution as indicated in Chapter 4, figure 4.3. For the purpose of this chapter, we are interested to examine how high performing firms differ from lower performing firms in the use of alliance mechanisms. Therefore, we created a dichotomous dummy variable, which defines high performing firms to have a success rate of $61-100 \%$, while other firms score $0-60 \%$.

We inquired about the size of firms' alliance portfolios (our independent variable) as the number of simultaneous operational alliances in 2007. In particular, our variable has three categories, with the following distribution, as depicted in table 6.2. 
Table 6.2: Distribution of alliance portfolio size in sample

\begin{tabular}{l|l|l}
\hline Alliance portfolio size & Frequency & Valid percentage \\
\hline small size (1-5 alliances) & 54 & 32 \\
\hline medium size (6-15 alliances) & 69 & 41 \\
\hline large size (more than 16 alliances & 46 & 27 \\
\hline Total & 169 & 100 \\
\hline
\end{tabular}

In order to investigate how firms manage alliance portfolios, our survey asked companies to indicate whether they used any of the thirty-four alliance mechanisms depicted in table 3.3 (Chapter 3). Each of these mechanisms is measured as a dichotomous dummy variable (yes versus no) and represent our independent variables. Therefore, companies can score up to a maximum of thirty-four alliance mechanisms in total. Hence, our survey provides us information on which alliance mechanisms companies use as well as the overall number of alliance mechanisms adopted by companies.

Furthermore, we are interested in the types of alliance mechanisms used by companies in our sample. In Chapter 3, we categorised these thirty-four alliance mechanisms into three conceptual groups according to the main purposes these mechanisms serve in alliance capability building (see table 3.3). The first group of alliance mechanism is associated with augmenting and transferring alliance-relevant knowledge throughout the organisation. The second group of alliance mechanisms is associated with building an alliance infrastructure and alliance functions. The third group of alliance mechanisms presents standardised processes that guide the day-to-day management of alliances.

Accordingly, we create three index variables that each represents a category of alliance mechanisms. Each of these index variables consists of the sum of the corresponding alliance mechanisms. 
The first index variable is called 'knowledge_mechanisms' and is the sum of the following alliance mechanisms (10 mechanisms in total):

- Competency framework

- Culture program

- Intercultural training

- In-house knowledge

- Alliance management

- development program
- Formal experience exchanges

- Best practices

- External alliance training

- In-house alliance training

- Intranet

Our second index variable is called 'infrastructure' and is the sum of the following alliance mechanisms (12 mechanisms in total):

- Alliance department

- Alliance managers

- Alliance specialist

- Vice president

- Local alliance managers

- Gatekeeper
- Rewards for business managers

- Rewards for alliance managers

- Mediators

- Financial experts

- Legal experts

- Consultants

Our third index variable is called 'stand_processes' and is the sum of the following alliance mechanisms (12 mechanisms in total):

- Individual evaluation

- Joint evaluation

- Cross-alliance evaluation

- Alliance metrics

- Alliance handbook

- Standard partner selection program
- Approval processes

- Alliance database

- Partner program

- Partner portal

- Joint business planning

- Country specific policies

By means of these three index variables, we can assess how firms differ in the adoption of different types of alliance mechanisms, and whether the adoption of any of these types of mechanisms has an effect on firm-level alliance performance.

\subsubsection{Analytical techniques}

Our hypothesis postulates that with an expansion in alliance portfolio size, firms are likely to increase portfolio success by using mechanisms associated 
with standardised processes and infrastructure. To test this, we will firstly compare the usage of alliance mechanisms across different portfolio sizes by means of ANOVA testing. Next, by means of chi-square statistics we test which alliance mechanisms are most effective for different categories of alliance portfolio size.

To test whether different alliance mechanisms exhibit different marginal contributions to firms' alliance performance, we use chi-square based statistics (cross-tabulations) to examine which alliance mechanisms are positively associated to a high alliance performance rate. The reason why we choose chi-square statistics rather than regression analysis is that a preliminary testing of our dataset revealed that our thirty-four alliance mechanisms are correlated to each other, that is, the use of one alliance mechanisms is associated with the use of other alliance mechanisms. To test all thirty-four alliance mechanisms simultaneously in the same regression model would distort our results, as the multicollinearity does not allow us to differentiate the effects of one alliance mechanism from the other.

\subsection{Analysis and Results}

To investigate which alliance mechanisms are most effective given the size of the portfolio, we first examine whether and how firms with different alliance portfolio sizes differ in the usage of alliance mechanisms. Running an ANOVA test reveals that firms with larger alliance portfolios use significantly more alliance mechanisms overall than firms with smaller alliance portfolios (see table 6.3). As depicted in table 6.3, the F-value is highly significant, indicating that at least one of the size categories differs significantly from the others. ${ }^{27}$ Therefore, we can state that firms with larger alliance portfolios use significantly more alliance mechanisms overall than firms with smaller alliance portfolios.

\footnotetext{
${ }^{27}$ The test results of the 'Levene test for homogeneity of variances' further confirm that the variance for the three categories of size is equal and the assumption is justified
} 
Table 6.3: Average adoption by absolute number of alliance mechanisms per alliance portfolio size category (ANOVA)

\begin{tabular}{l|c|c|c|l}
\hline & $\begin{array}{c}\text { small size } \\
\mathbf{( 1 - 5} \text { alliances) }\end{array}$ & $\begin{array}{c}\text { medium size } \\
\mathbf{( 6 - 1 5} \text { alliances })\end{array}$ & $\begin{array}{c}\text { large size } \\
\mathbf{( 1 6 + \text { alliances } )}\end{array}$ & F-value \\
\hline $\begin{array}{l}\text { number of mechanisms } \\
\text { adopted }\end{array}$ & $15,4(7,6)$ & $17,4(6,4)$ & $19(6,2)$ & $3,795^{* *}$ \\
\hline $\mathrm{N}$ & 54 & 69 & 46 & \\
\hline
\end{tabular}

There are 34 alliance mechanisms in total;

$\mathrm{N}$ total $=169$ companies

Cells provide means (in absolute numbers) and standard deviation for the number of alliance mechanisms adopted. ${ }^{*} \mathrm{p}<0.10 ;{ }^{* *} \mathrm{p}<0.05 ;{ }^{* * *} \mathrm{p}<0.01$

Furthermore, we examine whether firms with different sizes of alliance portfolios differ in the overall adoption of knowledge mechanisms, standardised processes and alliance functions. This question is important, as we hypothesised that firms with larger alliance portfolios are in need of a greater number of alliance infrastructure, functions and standardised processes. Table 6.4 exhibits the adoption of these three categories of mechanisms by firms with small, medium and large size portfolios. The measure to test this is the average number of alliance mechanisms for the categories 'knowledge transfer mechanisms', 'infrastructure \& functions' and 'standardised processes' per alliance portfolio size category.

Table 6.4: Average adoption of alliance mechanisms types per alliance portfolio size category (ANOVA)

\begin{tabular}{l|l|l|l|l}
\hline & $\begin{array}{l}\text { small size } \\
\mathbf{( 1 - 5} \text { alliances })\end{array}$ & $\begin{array}{l}\text { medium size } \\
\mathbf{( 6 - 1 5} \text { alliances) }\end{array}$ & $\begin{array}{l}\text { large size } \\
\mathbf{( 1 6 + \text { alliances } )}\end{array}$ & F-value \\
\hline knowledge transfer mech. & $4,8(2,7)$ & $4,4(2,5)$ & $4,6(2,5)$ &, 157 \\
\hline infrastructure \& functions & $4,6(2,9)$ & $5,6(2,2)$ & $6,3(2,1)$ & $6,56^{* * *}$ \\
\hline standardised processes & $6,5(3,1)$ & $7,3(2,9)$ & $8,1(2,8)$ & $3,59^{* *}$ \\
\hline $\mathrm{N}$ & 54 & 69 & 46 & \\
\hline
\end{tabular}

$\mathrm{N}$ total $=169$ companies

Cells provide means (in absolute numbers) and standard deviation for the number of alliance mechanisms adopted. ${ }^{*} \mathrm{p}<0.10 ;{ }^{* *} \mathrm{p}<0.05 ;{ }^{* * *} \mathrm{p}<0.01$

As depicted in table 6.4, firms with different alliance portfolio sizes do not significantly differ in the number of knowledge transfer mechanisms used. Regardless of alliance portfolio size, firms seem to use on average around four knowledge mechanisms (i.e. four out of ten knowledge transfer mecha- 
nisms in total). However, there are significant differences in the number of mechanisms used associated with infrastructure \& functions across different alliance portfolio sizes. While firms with small portfolio sizes use on average 4,6 mechanisms, firms with large sized alliance portfolio use on average 6,3 mechanisms associated with infrastructure and function (i.e. out of twelve mechanisms in total). The same holds for the usage of standardised processes, where firms with large portfolios use significantly more standardised processes than firms with smaller portfolios (i.e. out of twelve standardised processes in total).

Thus, this finding is in line with our expectation that alliance infrastructure, functions and standardised processes gain importance as the alliance portfolio size increases.

The question arises, whether these mechanisms are in fact effective to raise the performance of the portfolio. Therefore, to test which alliance mechanism are effective, we inspect each portfolio size category separately. In particular, we examine which alliance mechanisms are associated with high performing firms as compared to low performing firms, given the size of the portfolio.

Notably, a chi-square test reveals that there is no significant relationship between firms' alliance portfolio size per se and level of alliance portfolio performance. This fact eases our interpretation on which alliance mechanisms are most effective, as size does not influence the relationship. Table 6.5 summarizes which alliance mechanisms are most effective given the size of the portfolio. The outer left column indicates the category of portfolio size. Effective alliance mechanisms are presented according to their underlying purpose in the alliance capability development process (knowledge transfer, infrastructure, standardised processes). This way, we can better illustrate what kind of alliance mechanisms is most effective given the size of the portfolio. In particular:

Firms with small alliance portfolios are more likely to attain high performance rates when employing individual evaluation techniques, alliance metrics and approval processes. These mechanisms are standardised processes that particularly address tasks at the individual alliance management level. This finding confirms our expectation that firms with small portfolios are in little need to address portfolio relevant issues, but will concentrate on the tasks that arise on the individual alliance management level. 
Firms with medium sized alliance portfolios are more likely to attain high performance rates by using standardised processes specifically geared to the management of alliance portfolios such as joint evaluation, cross-alliance evaluations and joint business planning. Furthermore, use of knowledge augmentation mechanisms such as in-house alliance trainings and alliance management development programs help the firm to acquire and disseminate the necessary knowledge on alliance portfolio management. Moreover, creating alliance functions such as alliance managers and vice presidents as well as the employment of consultants, alliance specialists are crucial to ensure the effective application of alliance portfolio management knowledge, and hence to raise performance rates.

Firms with large alliance portfolios are more likely to attain high performance rates when emphasising knowledge mechanisms such as in-house alliance trainings, best practices and cultural programs. Finally, the use of consultants proves highly effective in raising the performance rates of large alliance portfolios.

Table 6.5: Critical success factors for alliance portfolio performance

\begin{tabular}{l|l|l}
\hline $\begin{array}{l}\text { small } \\
\text { alliance } \\
\text { portfolios }\end{array}$ & $\begin{array}{l}\text { standardised } \\
\text { processes } \\
\text { knowledge } \\
\text { mechanisms } \\
\text { alliance functions \& } \\
\text { infrastructure }\end{array}$ & $\begin{array}{l}\text { individual evaluation, alliance metrics, approval } \\
\text { processes }\end{array}$ \\
\hline $\begin{array}{l}\text { standardised } \\
\text { processes } \\
\text { knowledge } \\
\text { mechanisms }\end{array}$ & $\begin{array}{l}\text { joint evaluations, cross-alliance evaluations, joint } \\
\text { business planning } \\
\text { in-house alliance trainings, in-house knowledge, } \\
\text { alliance } \\
\text { portfolios }\end{array}$ & $\begin{array}{l}\text { alliance management development programs, } \\
\text { competency framework } \\
\text { consultants, alliance specialists, alliance manag- } \\
\text { ers, vice president of alliances }\end{array}$ \\
\hline $\begin{array}{l}\text { infrastructure } \\
\text { alliance } \\
\text { portfolios }\end{array}$ & $\begin{array}{l}\text { standardised processes } \\
\text { knowledge mechanisms }\end{array}$ & $\begin{array}{l}\text { in-house alliance training, best practices, culture } \\
\text { programs }\end{array}$ \\
& $\begin{array}{l}\text { alliance functions \& } \\
\text { infrastructure }\end{array}$ & \begin{tabular}{l} 
Consultants \\
\hline
\end{tabular}
\end{tabular}




\subsection{Discussion and Conclusion}

Given the trend towards establishing large alliance portfolios and the heterogeneity in alliance portfolio performance rates, the question how to manage alliance portfolios successfully has become vital. Alliance capability literature highlights the importance of building alliance capability that is specifically geared to address the challenges at the portfolio level of alliance management. However, so far, the conceptual construct of alliance portfolio capability as well as research into the underlying alliance mechanisms of alliance portfolio capability have remained scarce. Our aim has been to extend current research by examining how firms can successfully build alliance capability that supports the management of alliance portfolios. To this end, we reviewed state-of-the-art literature on alliance management in order to derive a differentiation of alliance capability on the individual versus portfolio level of alliance management. We then discussed which alliance mechanisms are likely to be effective in building alliance portfolio capability given the size of the alliance portfolio. In particular, we expected that with an increase in alliance portfolio size, firms make significantly more use of mechanisms associated with standardised processes and infrastructure in order to address portfolio relevant issues such as portfolio coordination and monitoring.

Our empirical results strongly confirm our hypothesis. First of all, we note that with an expansion in alliance portfolio size, firms use significantly more standardised processes as well as alliance infrastructure and functions. Noteworthy, the usage of knowledge transfer mechanisms seems to be independent of alliance portfolio size. Examples of knowledge transfer mechanisms are training programs intended to disseminate alliance management know-how throughout the organisation. A possible explanation why knowledge transfer mechanisms are independent of alliance portfolio size may be due to the fact that these mechanisms act as a 'qualifier', i.e. there are a basic requirement that is necessary to develop alliance capability. Therefore, firms use knowledge transfer mechanisms independent of the size of the alliance portfolio.

Secondly, we examined each size category separately and established which alliance mechanisms are most effective in raising portfolio performance. 
We found that firms with small alliance portfolios solely use three standardised processes to raise performance level. This is noteworthy, given that firms with small alliance portfolio use fifteen alliance mechanisms on average. As it appears, selecting a few chosen mechanisms geared to the management of individual alliances are most effective in raising the performance of the small portfolio.

Moreover, interesting to note is the finding that firms with large alliance portfolios raise performance by using a number of knowledge mechanisms and alliance functions, but not standardised processes. Firms with large alliance portfolios are more likely to attain high performance rates when emphasising knowledge mechanisms such as in-house alliance trainings, best practices and cultural programs. The latter two are exactly in line with Heimeriks et al. (2008), emphasising the importance of adopting a more structured and deliberate approach to accumulating and disseminating alliance related knowledge.

These findings have important theoretical implications. Firstly, considering the management of large alliance portfolios we have established the important role of knowledge transfer mechanisms and alliance functions. Notably, no standardised processes are found effective. The reasoning might be as follows. Firms with a large portfolio experience a great need to build alliance capability in order to cope with the numerous challenges that accompany an expansion in portfolio size. Here for, training programs disseminate valuable alliance management knowledge. With an expansion in portfolio size, alliance infrastructure and functions gain importance as the firm experiences a greater need for coordination of the portfolio. However, standardised processes become less important as the portfolio grows, probably because a large portfolio implies a variety in alliance partners and alliance types, which cannot be managed with standardised processes but need individual attention. Therefore, our results strengthen the theoretical understanding of the importance of knowledge accumulation (knowledge transfer mechanisms) and knowledge application (alliance infrastructure \& functions) in building alliance capability.

Notably, our results illustrate a difference in which alliance mechanisms are in use versus which alliance mechanisms prove to be effective. For instance, considering the average number of alliance mechanism used, we find that 
firms with small portfolios use about fifteen alliance mechanisms on average, whereas only three mechanisms prove to be effective. A possible explanations for this gap might be due to the inexperience of firms who presume 'the more alliance mechanisms, the better'. Moreover, we find that with an expansion in portfolio size, firms rely more extensively on standardised processes. However, our analysis shows that standardised processes do raise performance of small and medium but not large sized portfolios. We suspect that many firms are unaware of the specific managerial challenges that arise with a particular size of the alliance portfolio, and therefore adopt as many alliance mechanisms as possible without further consideration whether these mechanisms are appropriate.

For these reason, a study such as ours can contribute to advancing the knowledge on how to build alliance portfolio capability. We have illustrated the multifaceted construct of alliance capability, showing that alliance capability can be built by means of many different alliance mechanisms. Our results illustrate that the effectiveness of alliance mechanisms is contextrelated, i.e. dependent on the size of the alliance portfolio. Herewith we have strengthened extant research on alliance portfolio capability, confirming the importance of differentiating alliance capability on the individual and portfolio level. 



\section{Chapter 7 Managing Alliance Portfolio DIVERSITY}

\subsection{Introduction}

Accompanying the trend towards forming large alliance portfolios (Lavie, 2007) is the remarkable rise in the degree of complexity that characterizes many of today's alliance portfolios. To serve a multitude of purposes, firms do not only engage with partner firms from different countries, but also collaborate with firms across industries and along the value chain. The results are highly complex configurations of alliance portfolios that encompass various functional activities (e.g. manufacturing, marketing, R\&D alliances) and span across national and industry borders.

So far, alliance literature has examined the phenomenon of alliance portfolio diversity (i.e. the variety in alliance partners and functional activities encompassed in alliance portfolios) with regard to its implications for firm performance and innovativeness (Beckman and Haunschild, 2002; Bae and Gargiulo, 2004; Baum, Calabrese and Silver, 2000; George, Zahra, Wheatley and Khan, 2001; Rowley, Behrens and Krackhardt, 2000, Stuart, 2000). Depending on the theoretical lens adopted, diversity in alliance portfolios is suggested to be either beneficial or harmful.

A neglected topic in extant alliance literature is the issue of identifying and addressing the managerial challenges that arise in diverse alliance portfolios. Our previous analysis of the managerial challenges of alliance portfolio size (Chapter 6) indicated that an increase in the number of alliance relationships in the portfolio is bound to increase the managerial burden. Hence, the question arises whether an increase in the diversity of alliance partners and functional activities is likely to raise additional managerial challenges that need to be addressed effectively. To our knowledge, this issue has received little attention from alliance management literature.

Therefore, this chapter is dedicated to examining which managerial challenges arise with alliance portfolio diversity and how firms can manage 
these challenges most effectively. Investigating how firms can effectively manage alliance portfolio diversity will provide scholars and practitioners with the indispensable insight on how firms can raise the performance of their alliance portfolios successfully.

Given the nascent stage of this research topic, we first, we need to gain a comprehensive understanding of the nature of alliance portfolio diversity, understanding what motivates firms to create diverse alliance portfolios. To this end, we draw on social network and transaction cost theory to compare the merits and drawbacks of diversity in alliance partners and functional activities.

Second, in order to derive how firms can deal with these managerial challenges most effectively, we refer to alliance capability literature. From an alliance capability view, the successful management of alliances is suggested to depend on the firm's ability to learn about alliance management and to translate these lessons into applicable, standardised alliance management practices. Especially with regard to the management of alliance portfolios, alliance capability scholars have suggested various alliance management mechanisms to increase alliance portfolio performance. However, the predominant focus of extant alliance capability research has been on testing which alliance management practices are particularly effective in managing a large number of alliances simultaneously; that is, the focus has been on the size related aspect of alliance portfolio management (Duysters et al., 1999; Heimeriks et al., 2008; see Chapter 6). What managerial challenges arise with alliance portfolio diversity and how firms can effectively build alliance capability that addresses these issues has not been examined to date.

Therefore, by combining the insights from various streams of alliance theories and research we review the motivations underlying diverse alliance portfolios and derive which managerial challenges are likely to arise. By means of an exhaustive empirical analysis, we test which alliance mechanisms prove to be most effective in addressing these managerial challenges and thus raise alliance portfolio performance. 


\subsection{Understanding alliance portfolio diversity}

In the following, we will examine the multifaceted construct of alliance portfolio diversity and provide theoretical perspectives on the motivations, merits and drawbacks of introducing diversity to alliance portfolios.

\subsubsection{Types of alliance portfolio diversity}

When creating a portfolio of alliances, the firm faces inter alia two important issues regarding the configuration of the portfolio, namely which firms should we ally with and what range of activities should be performed within the alliance portfolio (i.e. R\&D, marketing, manufacturing alliances).

First, regarding the question of partner selection, a firm can decide whether to ally with similar or different types of partners. For example, a portfolio can consist of partners from the same industry or encompass partners from a variety of different industries. Whether a firm tends to vary or converge on the types of alliance partners in its portfolio, determines the degree of alliance partner diversity. In alliance research, different types of alliance partner diversity have been examined, such as diversity in partners' national backgrounds (reference), sectoral backgrounds (Hoffmann, 2007), resources (Goerzen and Beamish 2005) or technology (Sampson, 2007; Lee, 2007).

Second, a firm can decide about the range of different functional activities within the alliance portfolio. As alliances can be used for all functional activities in a firm's value chain, we often see alliances being established for the purposes of research and development, marketing, production or distribution. Whether a firm tends to vary or converge on the types of functional activities within the portfolio determines the degree of functional diversity. For instance, a maximum of functional diversity is attained, when the firm decides to pursue all functional activities of its value chain by means of alliances, that is, entering into $R \& D$, marketing, manufacturing, distribution, supplier and customer alliances simultaneously. Eventually, the choices regarding the variety in partner firms and functional activities result in the overall configuration of the alliance portfolio.

To simplify, based on the two dimensions of partner diversity and functional diversity, the firm can attain four different types of alliance portfolio configurations, as depicted in figure 7.1. 
In quadrant $\mathrm{A}$, the alliance portfolio is characterised by a low degree in partner diversity and functional diversity; that is, the firm focuses on a single type of alliance partner and a single type of functional activity.

In quadrant $B$, the alliance portfolio is characterised by a high degree of partner diversity but only a low degree in functional diversity; that is, while the firms allies with a variety of partner types, the focus is on a single type of functional activity.

In quadrant $C$, the alliance portfolio is characterised by a high degree of diversity in both partner firms and functional activities.

In quadrant $\mathrm{D}$, the alliance portfolio is characterised by a low degree in partner diversity but a high degree in functional diversity; that is, while the firm focuses on a single type of alliance partner, it engages in a variety of different functional activities.

While our typology in figure 7.1 only illustrates a simple representation of alliance portfolio configurations, the choices a firm makes regarding the degrees of partner and functional diversity are likely to resemble one of these four quadrants (Jiang, Tao and Santoro, 2010; and Bruyaka, 200?).

Figure 7.1: Typology of alliance portfolio configurations

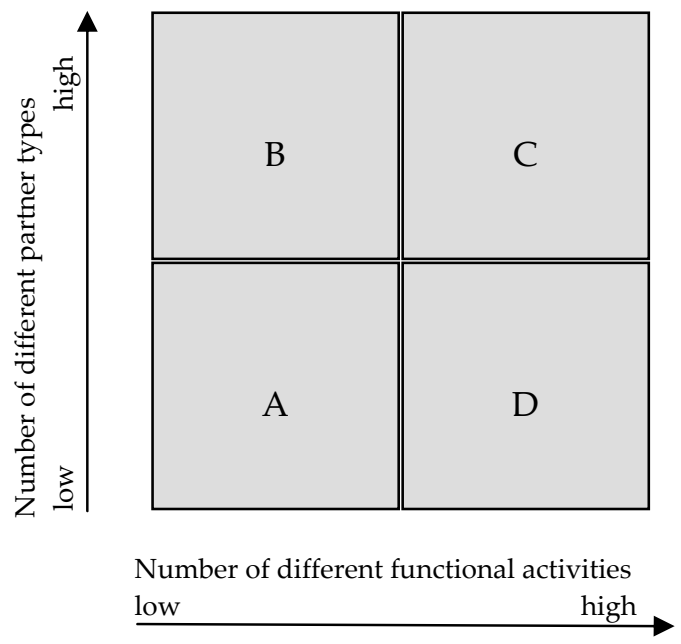

Source: adapted from Jiang, Tao and Santoro (2010) 


\subsubsection{Theoretical perspectives on the merits and drawbacks of alliance portfolio diversity}

Alliance scholars draw a link between a firm's overall business strategy and a firm's configuration of its alliance portfolio (diverse versus non-diverse portfolio). According to Hoffmann (2005), a firm's business strategy sets the goals for all alliances in the portfolio (e.g. entering a foreign market or developing a new technology) and hence influences the number and types of alliances that are entered in the course of the firm's strategy. Such an 'alliance portfolio strategy' is thus derived from a firm's overall strategy and hence determines whether a firm creates a diverse alliance portfolio.

Depending on the theoretical lens adopted, we find numerous arguments against and in favour of creating a diverse alliance portfolio. First, we review the arguments regarding diversity in alliance partners; followed by arguments regarding diversity in functional activities.

\section{Merits and drawbacks of partner diversity}

Largely drawing on social network theory, scholars advertise the benefits of maintaining variance in partner firms. While the number of direct alliance relationships determines the overall size of the alliance portfolio and thus the quantity of information and resources the firm can access by virtue of its alliances, the variety of alliance partners determines the diversity of sources of information and resources. Increasing the variance in partner firms implies more breadth in the search for novel opportunities and provides for multiple options to gain access to unique and non-redundant information and resources (Beckman and Haunschild, 2002; Kattila and Ahuja, 2000). Redundancy is defined as the contextual overlap in information and resources (Koka and Prescott, 2002; McEvily and Zaheer, 1999). A high degree of redundancy among alliance partners suggests no gain in information, as each partner stems from a similar knowledge or technological background. A maximum degree of variety, however, provides for the richest possible distribution of information as the focal firm allies with partners from distinct backgrounds (e.g. international partners, inter-industry partners). Hence, a low degree of redundant relationships in the alliance portfolio ensures diversity in information and resources, which the focal firm can access by virtue of its alliance partners. Further, a low degree of redundant relationships in the alliance portfolio leads to structural holes between the focal firm's partners, which the firm can exploit to gain control advantages (Burt, 1992). 
Studies have shown that variance in partners is generally associated with less redundancy in information and hence greater creativity and innovativeness (Sampson, 2007; Harrison and Klein, 2007; Baum, Calabrese and Silverman, 2000).

However, a homogenous alliance portfolio with somewhat similar alliance partners has its advantages, too. Referring to the argument of absorptive capacity, several scholars emphasise the benefits of partner similarity. Research on inter-organizational learning (e.g., Argyris and Schön, 1978, Fiol and Lyles, 1985; Dosi 1988; Moingeon and Edmondson, 1996) has revealed that firms are more likely to acquire a new skill or capability when these are closely associated with an existing competence base (Cohen and Levinthal, 1990). Therefore, forming alliance relationships with similar partners (e.g., same country, industry, technological background as focal firm) provides the benefit of 'familiarity' when it comes to absorbing the knowledge exchanged between partners (Beckman and Haunschild, 2002). For instance, studies have shown that similarity in partners' technological capabilities and knowledge bases is found to enhance inter-partner learning (Lane and Lubatkin, 1998), increase firms' patenting rate (Ahuja 2000) and positively affect post-alliance firm development (Mowery, Oxley and Silverman, 1996). To summarize, regarding the choice whether to engage with similar or dissimilar types of alliance partners, the focal firm faces the trade-off between the richness of information obtained from similar partner firms and, on the other hand, the diversity of information and resources obtained from dissimilar firms.

Furthermore, diversity in alliance partners is often associated with additional managerial burden. To illustrate the many difficulties that managers experience in alliance portfolios with diverse partners, we briefly refer to two common examples of partner diversity, namely when firms include partner firms from different national and industry backgrounds.

Including foreign partners into the alliance portfolio allows the firm to leverage their competitive advantage in foreign markets. Allying with foreign partners is suggested to allow for faster market entry, less investment required and facilitates learning about the foreign markets customs (Glaister and Buckley, 1996). Notwithstanding the many potential benefits, engaging with geographic diverse alliance partners generates a multitude of risks that are less likely to occur within alliance portfolios consisting of purely domes- 
tic alliances. In particular, the most commonly reported drawbacks of geographical diverse alliance portfolios include (1) the risk of undesirable resource spillovers and misappropriation of value by foreign partners (Hamel, 1991; Lavie, 2006); (2) the risk of information asymmetries exploitation by foreign partner possessing superior knowledge of local business and regulatory environment (Yan and Gray, 1994); (3) increased alliance governance costs while the potential share of proceeds is likely to decrease (Khanna et al., 1998); (4) differences in national cultures, institutional environments and value systems that severely limit the scope for familiarity, shared values, priorities and goals, hence hampering social exchanges and interfirm trust in alliances (Parkhe, 1991; Gulati, 1995; Lane and Beamish, 1990); and (5) the problem of 'double layered acculturation', where adjustments to both a foreign country and corporate culture is required (Barkema et al., 1996). These and more barriers are frequently found in international alliances, leading to relational ambiguities and mistrusts that impair learning (Parkhe, 1991; Simonin, 1999), eventually reducing the effectiveness of collaboration with foreign partners (Barkema et al., 1996; Kumar and Nti, 1998; Lane and Lubatkin, 1998; Pothukuchi et al. 2002).

To broaden their set of competencies, firms often include alliance partners from different industry backgrounds. Firms from different industry backgrounds are likely to possess distinct resource endowments with specific, idiosyncratic skills and capabilities (Nelson 1991; Rumelt, Schendel and Teece, 1991) that create significant learning opportunities (Hamel et al., 1989). Therefore, firms often choose to ally with firms from unrelated business fields as to access different knowledge and technology bases (Beckman and Haunschild, 2002) and eventually to combine complementary assts in order to pursue new business opportunities (Dussauge, Garette, Mitchell, 2000; Santoro and McGill, 2005). According to Santoro and McGill (2005) inter-industry alliances allow the focal firm to gain access to different knowledge and technology bases. However, such exposure to unfamiliar grounds does not allow for a similar background for knowledge exchange. As Lane and Lubatkin (1998) point out, the firm's ability to internalise the partner's knowledge is greater when knowledge processing systems are similar.

Therefore, diversity in partner firms implies a variety in cultural, social, economical and political conditions, which on the one hand ensures access to a 
variety of sources of resources and information, and, on the hand however generates managerial problems associated with unfamiliarity (Roth and O'Donnell, 1996). Hence, the managerial challenges that arise with diversity in alliance partners mainly regard high search costs associated with finding a suitable alliance partner outside the focal firm's known sphere of contacts (Rangan 2000). Here the unfamiliarity with and lack of reputation of potential alliance partners increases the probability of adverse partner selection (Goerzen and Beamish, 2005).

\section{Merits and drawbacks of functional diversity}

Essentially, alliances with suppliers or customers are established in order to gain economics of scale or scope (Das and Teng, 2000). Marketing alliances are often used to leverage existing resources (e.g. exploitative alliances), while R\&D alliances allow the partner firms to broaden their technological competence base (e.g. explorative alliances). Often, firms decide to engage in both explorative and exploitative alliance strategies as to secure current and future viability (March, 1991).

This logic is in line with 'modern portfolio theory' (references) and 'realoption theory' (references) where scholars draw an analogy between a firm's portfolio of financial assets and alliance relationships (references). Including a variety of functional activities, the firm can diversify away risks associated with putting all 'eggs in the same basket'. Instead, an alliance portfolio with a variety of different functional activities allows the firms to hedge against technology and market risks. Research has investigated the implications of functional diversity in alliance portfolios. For instance, studies suggest that firms with different types of alliances (R\&D, marketing, manufacturing) in their portfolios are more likely to be central in industry networks and therefore enjoy higher growth rates (Powell, Koput and Smith-Doerr, 1996) as well as to benefit from higher firm level performance (Baum et al., 2000). However, maintaining a high diversity in functional activities is also associated with increased administrative and managerial burden and risk of coordination errors (Williamson, 1985). Adding a variety in functional activities to the alliance portfolio is expected to negatively affect alliance performance due to the increased burden on managing the alliance portfolio (Hoang, 2001). Given the limited managerial or administrative capacity each firm has, the extent of functional diversity that can be effectively managed is limited. Moreover, the logic of 'bounded rationality' implies that firms are restricted 
in their ability to process complex decision-making situations. Hence, an alliance portfolio with a variety of functional activities carried out simultaneously requires greater information processing than a portfolio consisting of similar activities (Dess and Beard, 1984). Furthermore, diversity in functional activities leads to a complexity of resources within a portfolio, promoting inter-organizational dependencies (Aldrich, 1999).

\section{Discussion}

Our review of alliance portfolio diversity literature has shown that firms face a number of arguments in favour and against creating diverse alliance portfolios. The main rationale for creating a diverse portfolio of alliances appears to be the benefits of accessing various different sources of knowledge and resources by means of establishing alliances with different partners and functional activities.

Our main point of discussion regards the fact that extant studies on the topic of alliance portfolio diversity focus on the performance implications of diversity without examining how firms can manage alliance portfolio diversity effectively. To illustrate, the typical research question in alliance portfolio diversity literature investigates what effect a certain type of diversity (diversity in partners' technology, industrial background) has on firm-level performance or innovativeness. That is, while we have exhaustive accounts of the various challenges and merits that arise with alliance portfolio diversity, these studies do not provide insights on how to address these challenges effectively. In order to understand how firms can effectively manage alliance portfolio diversity, we refer to alliance capability literature in the next section.

\subsection{Managing alliance portfolio diversity}

Over the last decade, the management of alliance portfolios has become an important research focus in alliance literature (Wassmer, 2010). Drawing on dynamic capability and organisational learning theories, an emerging group of scholars has investigated how firms learn to manage alliances and build alliance capability (Anand and Khannna, 2000; Kale et al. 2002). Research has shown that accumulating alliance experience is decisive for the development of alliance capability (Anand and Khanna, 2000; Hoang and Rothaermel, 
2005). Some scholars thereby suggested that in order to build alliance capability and to capitalize on its accumulated alliance experience, the firm needs to develop organizational mechanisms that capture, internalise, and apply alliance experience in the form of accessible knowledge throughout the organization (Kale et al. 2002; Kale and Singh, 2007). More recently, scholars have voiced the need to differentiate alliance capability on the individual versus portfolio level of alliance management (Hoffmann, 2005; Sarkar et al., 2009). Alliance portfolio capability is suggested to present a firm's "organizational ability to manage a comprehensive alliance portfolio successfully" (Hoffmann, 2005:123) by specifically addressing the formation, development and integration of alliance portfolios (Sarkar et al., 2009). Important areas of alliance portfolio management include developing alliance portfolio strategy and selecting partners that fit the overall goal of the alliance portfolio, coordination and prioritization of resources, as well as monitoring and assessing the performance of the alliance portfolio as an entirety (Sarkar et al., 2009; Hoffmann, 2005; Bamford and Ernst, 2002; Fricke and Shenkar, 2000). To this end, alliance scholars have suggested numerous alliance management mechanisms especially geared to support the tasks of alliance portfolio management.

For instance, our empirical analysis suggested that with an expansion in alliance portfolio size, the need for knowledge augmentation and transfer mechanisms significantly increases, as the firm recognizes the need to develop alliance portfolio capability to manage its many alliances. Especially training programs for alliance staff are found to significantly increase alliance portfolio performance. Moreover, with an expansion in portfolio size, alliance infrastructure and staff gain importance as the firm experiences a greater need for coordination of the portfolio. However, standardised processes become less important as the portfolio grows, as a large portfolio implies a variety in alliance partners and alliance types, which cannot be managed with standardised processes but need individual attention (please refer to Chapter 6).

Important to note, our review of state-of-the-art literature in Chapter 6 indicated that extant alliance capability literature is focussed on the size related aspect of alliance portfolio management. What managerial challenges arise with alliance portfolio diversity and how firms can effectively build alliance capability that addresses these issues has not been examined to date by an alliance capability perspective. 
Instead we find many indications about potential problems and managerial challenges of alliance portfolio diversity in non-alliance management literature (as reviewed in section 7.2). However, as we have pointed out earlier, this stream of literature does not indicate how firms can actually manage the challenges that arise in diverse portfolio.

Hence, little is known about which alliance management mechanisms are most effective in addressing the challenges that arise in alliance portfolios with diverse types of partner firms and functional activities. Therefore, we like to extend the current work on alliance portfolio management by examining which alliance mechanisms are most effective in addressing alliance portfolio diversity.

In particular, we are interested to investigate how to manage different types of alliance portfolio configurations in terms of diversity. That is, referring to our figure 7.1, firms can create alliance portfolios with

A) no diversity in types of partners firms and functional activities

B) different types of partner firms but similar type of functional activity

C) different types of partner firms and functional activities

D) similar type of partner firms but different types of functional activities

As we are interested in a portfolio perspective of alliance management, we do not examine partner diversity or functional diversity separately, but regard these as dimensions of alliance portfolio configuration. Therefore, we examine how firms can manage different types of alliance portfolio configurations (A, $\mathrm{B}, \mathrm{C}$ and $\mathrm{D})$.

To research our question on successful portfolio management, the remaining of this chapter is dedicated to generate empirical evidence on how firms configure alliances portfolios and which alliance mechanisms are most effective in enhancing alliance performance given the type of alliance portfolio diversity. 


\subsection{Method: variables and analytical techniques}

To understand how firms can successfully manage alliance portfolios, our literature review has indicated the importance of building alliance capability in raising the performance of diverse alliance portfolios. In the following, we are interested to examine empirically which alliance mechanisms are most effective in raising performance rate of different types of alliance portfolio configurations.

To examine this issue, our survey included a number of questions on this matter, e.g. inquiring respondents about their companies' configuration of alliance portfolios, alliance performance level, and the use of alliance mechanisms. The details on our survey and sample are provided in Chapter 4 'Methodology'; the corresponding questionnaire is included in Appendix 1. In the following, we provide a brief overview of the variables and analytical techniques used to investigate our research questions.

\subsubsection{Variables}

Our aim is to investigate how firms can increase firm-level alliance portfolio performance; hence, alliance performance is our dependent variable. To measure alliance performance, we relied upon the managerial assessments of firm-level alliance performance, measuring alliance success as the percentage of alliances where the firm's initial goals were realized (e.g. Kale, Dyer and Singh, 2002). Our original variable on alliance portfolio performance has five categories, with a distribution as indicated in Chapter 4, figure 4.3. For the purpose of this chapter, we are interested to examine how high performing firms differ from lower performing firms in the use of alliance mechanisms. Therefore, we created a dichotomous dummy variable, which defines high performing firms to have a success rate of $61-100 \%$, while other firms score $0-60 \%$.

In order to investigate how firms manage alliance portfolios, our survey asked companies to indicate whether they used any of the thirty-four alliance mechanisms depicted in table 3.3 (Chapter 3). Each of these mechanisms is measured as a dichotomous dummy variable (yes versus no) and represent independent variables. Therefore, companies can score up to a maximum of thirty-four alliance mechanisms in total. Hence, our survey 
provides us information on which alliance mechanisms companies use as well as the overall number of alliance mechanisms adopted by companies. Furthermore, we are interested in the types of alliance mechanisms used by companies in our sample. Therefore, we will re-use our three index variables, which we defined in Chapter 6, section 6.4.1.

- index variable 'knowledge_mechanisms' for the category of 'knowledge augmenting and transfer mechanisms

- index variable 'infrastructure' for the category of 'infrastructure and functions' mechanisms

- index variable 'stand_processes' for the category of 'standardised management processes'

In this chapter, we are interested to examine what different types of alliance portfolio configurations are used by the companies in our sample. Our questionnaire did not include a question regard this matter per se, rather we asked four questions, which taken together indicate which type of alliance portfolio diversity is adopted by the companies.

To examine the degree of functional diversity in companies' alliance portfolios, we asked companies to indicate how many different functional activities are included in their alliance portfolio simultaneously. As companies could indicate up to five functional activities, our variable has five categories, with a distribution as indicated by table 7.1.

At the minimum, a firm focuses on one single type of functional activity (20 $\%$ of all companies in our sample focus on one type of functional activity in their alliance portfolio only). At the maximum, a firm includes all five functional activities in its alliance portfolio simultaneously (i.e. R\&D, marketing, distribution, manufacturing and supplier alliances at the same time (in our sample, $12 \%$ of all companies have an alliance portfolio encompassing five functional activities simultaneously). 
Table 7.1: Distribution of functional activities in alliance portfolios

\begin{tabular}{l|l}
\hline number of functional activities types & valid percentage \\
\hline One type of functional activity & $20 \%$ \\
\hline Two types of functional activity & $24 \%$ \\
\hline Three types of functional activity & $22 \%$ \\
\hline Four types of functional activity & $22 \%$ \\
\hline Five types of functional activity & $12 \%$ \\
\hline
\end{tabular}

To examine the degree of partner diversity in companies' alliance portfolios, we asked companies to indicate how many different partner types are included in their alliance portfolio simultaneously. As companies could indicate up to three different partner types, our variable has three categories, with a distribution as indicated by table 7.2. These three different partner types include alliance partners from different national backgrounds, industry backgrounds or competitors.

At the minimum, a firm engages with only one type of alliance partner $(24 \%$ of all companies in our sample ally with one type of alliance partner only). At the maximum, a firm allies with three different types of alliance partners, i.e. allying with international partners, inter-industry partners and competitors at the same time (in our sample, $38 \%$ of all companies have an alliance portfolio encompassing three different partner types simultaneously).

Table 7.2: Distribution of partner diversity in alliance portfolios

\begin{tabular}{l|l}
\hline number of different partner firms & valid percentage \\
\hline one type of alliance partners & $24 \%$ \\
\hline two types of alliance partners & $38 \%$ \\
\hline three types of alliance partners & $38 \%$ \\
\hline
\end{tabular}

\subsubsection{Analytical techniques}

We use a number of multivariate statistics to test (1) what types of alliance portfolios are created by companies, (2) whether firms with different types of alliance portfolio configurations differ in their overall adoption and use of alliance mechanisms, and finally (3) which alliance mechanisms are most effective in raising performance levels of different alliance portfolio types.

Firstly, we are interested to examine how firms configure their alliance portfolios along the two dimensions of partner and functional diversity. Here we have two choices. We can create categories of alliance portfolio types manu- 
ally, that is by creating dummy variables that resemble the four categories of alliance portfolio configuration as depicted in figure 7.1. However, the drawback of this approach is that it does not reflect how companies actually configure their alliance portfolios in practice. Therefore, we choose to run a cluster analysis, a method that groups firms in our sample according to their type of alliance portfolio configuration. As a result, a cluster analysis identifies relatively homogenous groups of firms in terms of their portfolio configurations. A cluster analysis does not necessary generate four types of alliance firms (as in our conceptual model), but reflect the typical patterns of alliance portfolio configurations in our sample

Secondly, we investigate whether firms with different alliance portfolio types differ significantly in the overall adoption and usage of alliance mechanism. An ANOVA test will show if there are significant differences among types of firms.

Thirdly, we are interested in the effectiveness of alliance mechanisms for different types of alliance portfolios. Therefore, we examine each category of alliance portfolio configuration separately. By means of chi-square statistics, we test which alliance mechanisms are significantly associated to a high alliance portfolio performance given the type of alliance portfolio. The reason why we choose chi-square statistics rather than regression analysis is that a preliminary testing of our dataset revealed that our thirty-four alliance mechanisms are correlated to each other, that is, the use of one alliance mechanisms is associated with the use of other alliance mechanisms. To test all thirty-four alliance mechanisms simultaneously in the same regression model would distort our results, as the multicollinearity does not allow us to differentiate the effects of one alliance mechanism from the other. 


\subsection{Analysis and Results}

Firstly, we test which typical configurations of alliance portfolios exist in our dataset. We run a cluster analysis to determine how firms configure alliance portfolios along the dimensions of partner diversity and functional diversity. As laid out above, the degree of partner diversity ranges from one to three, indicating that an alliance portfolio can consists either of one, two or three types of alliances partners simultaneously. Degree of functional diversity ranges from one to five, indicating that an alliance portfolio can consists of one up to five functional activities simultaneously. The results of the cluster analysis are depicted in table 7.3. The values in the cells are the means for each variable within each final cluster.

Table 7.3: $\quad$ Final Cluster Centres

\begin{tabular}{l|l|l|l}
\hline & \multicolumn{4}{l}{ Cluster of firms } \\
\hline & A & B & C \\
\hline Degree of partner diversity & 1 & 1 & 3 \\
\hline Degree of functional diversity & 4 & 1 & 3 \\
\hline
\end{tabular}

In particular, we find that:

Companies in cluster A have alliance portfolios with one type of alliance partner and four different types of functional activities. Hence, this type of alliance portfolio is characterised by a low degree of partner diversity and a high degree of functional diversity Therefore, we label this type of alliance portfolios as 'low partner diversity $\mathcal{E}$ high functional diversity'.

Companies that belong to cluster $\mathbf{B}$ have alliance portfolio with one type of alliance partner and one type of functional activity. Hence, this type of alliance portfolio is characterised by low partner diversity and a low functional diversity. As the firm keeps diversity in both partners and functional activities to a minimum, we label this type of alliance portfolio as 'low partner diversity \& low functional diversity'.

Companies that belong to cluster $\mathbf{C}$ have alliance portfolios with three different types of alliance partners as well as three different types of functional activities. Hence, this type of alliance portfolio is characterised by a high degree of partner diversity as well as high functional diversity. As the firm 
maintains a high degree of diversity in both partner firms and functional activities, we label this type of alliance portfolio as 'high partner diversity $\mathcal{E}$ high functional diversity'.

Strikingly, we find only three typical patterns of alliance portfolio configurations as opposed to our initial four types (which we derived conceptually). The category of 'high partner diversity $\mathcal{E}$ low functional diversity is not represented in our cluster analysis, indicating that this category does not present a commonly adopted configuration within firms in our sample.

Summarising, table 7.4 shows the distribution of these three types of alliance portfolios in our sample. ${ }^{28}$

Table 7.4: Number of Cases in each Cluster

\begin{tabular}{l|l|l}
\hline \multirow{2}{*}{ Cluster } & low partner \& high functional diversity (Cluster A) & 33 \\
\cline { 2 - 3 } & low partner \& low functional diversity (Cluster B) & 61 \\
\cline { 2 - 3 } & high partner \& high functional diversity (Cluster C) & 79 \\
\hline Valid & & 173 \\
\hline Missing & & 2 \\
\hline
\end{tabular}

Secondly, we are interested to examine whether firms with different portfolio configurations differ in the overall number of alliance mechanisms adopted. By means of one-sided ANOVA (table 7.5), we find that firm high partner $\mathcal{E}$ high functional diversity' use significantly more alliance mechanisms overall than the other firms (F-value is significant at the $5 \%$ level).

Table 7.5: Average adoption of absolute number of alliance mechanisms (ANOVA)

\begin{tabular}{l|l|l|l|l}
\hline & $\begin{array}{l}\text { low partner E high } \\
\text { functional diversity }\end{array}$ & $\begin{array}{l}\text { low partner \& low } \\
\text { functional diversity }\end{array}$ & $\begin{array}{l}\text { high partner E high } \\
\text { functional diversity }\end{array}$ & F-value \\
\hline $\begin{array}{l}\text { overall number of } \\
\text { mechanisms }\end{array}$ & $16(6,6)$ & $15(6,9)$ & $18(6,9)$ & $2,60^{* *}$ \\
\hline $\mathrm{N}$ & 33 & 61 & 79 & \\
\hline
\end{tabular}

There are 34 alliance mechanisms in total; $\mathrm{N}=169$;

$\mathrm{N}$ total: 173 companies

Cells provide means (in absolute numbers) and standard deviation for the number of alliance mechanisms adopted. ${ }^{*} \mathrm{p}<0.10 ;{ }^{* *} \mathrm{p}<0.05 ;{ }^{* * *} \mathrm{p}<0.01$

Next, we use one-sided ANOVA to test whether firms with different portfolio configurations differ in the type of alliance mechanisms used. Regarding

${ }^{28}$ Supporting our findings, a relatively large $\mathrm{F}$ ratio in the ANOVA output indicate that we deal with a 'good' cluster analysis. 
the adoption of knowledge mechanisms, firms with different portfolio configurations use on average four knowledge mechanisms and hence do not significantly differ (the F-value is not significant). Similarly, regarding the adoption of infrastructure \& functions, firms with different portfolio configurations use on average 5 mechanisms and hence do not significantly differ (the F-value is not significant). However, there is a significant difference in the adoption of standardised processes across the three types of alliance portfolios, where firms with 'low partner $\mathcal{E}$ low functional diversity' use significantly less mechanisms than the other two portfolio types.

Table 7.6: Average adoption of categorised alliance mechanisms per alliance portfolio category (ANOVA)

\begin{tabular}{l|l|l|l|l}
\hline & $\begin{array}{l}\text { low partner \& high } \\
\text { functional diversity }\end{array}$ & $\begin{array}{l}\text { low partner \&low } \\
\text { functional diversity }\end{array}$ & $\begin{array}{l}\text { high partner \& high } \\
\text { functional diversity }\end{array}$ & F-value \\
\hline $\begin{array}{l}\text { knowledge } \\
\text { mechanisms }\end{array}$ & $4(2,5)$ & $4(2,5)$ & $4(2,5)$ & 0,87 \\
\hline $\begin{array}{l}\text { infrastructure \& } \\
\text { functions }\end{array}$ & $5(2,3)$ & $5(2,5)$ & $5(2,6)$ & 2,10 \\
\hline $\begin{array}{l}\text { standardised } \\
\text { processes }\end{array}$ & $7(2,7)$ & $6(3,1)$ & $7(3,0)$ & $2,87^{*}$ \\
\hline $\mathrm{N}$ & 33 & 61 & 79 & \\
\hline $\mathrm{N}$ & & &
\end{tabular}

$\mathrm{N}$ total: 173 companies

Cells provide means (in absolute numbers) and standard deviation for the number of alliance mechanisms adopted. ${ }^{*} \mathrm{p}<0.10 ;{ }^{* *} \mathrm{p}<0.05 ;{ }^{* * *} \mathrm{p}<0.01$

Thirdly, we are interested to examine which alliance mechanisms are most effective given a type of portfolio configuration. For this purpose, we inspect each type of alliance portfolio separately. We summarize our findings in table 7.7 below. In each column, we have indicated those alliance mechanisms that are effective in raising the performance level of the respective type of alliance portfolio. For instance, for firms that have a portfolio with a low partner $\mathcal{E}$ low functional diversity (third column) using alliance management development programs, individual and joint evaluation techniques is sufficient to raise performance level.

For firms in the first two categories, several alliance mechanisms are similarly important to raise performance level. The usage alliance metrics, crossalliance evaluation, joint evaluation, partner portals, cultural training and consultants increases the performance of both types of alliance portfolios. 
However, we can see other alliance mechanisms that are effective only for a particular alliance portfolio type.

For portfolios with low partner $\mathcal{E}$ high functional diversity, there is a higher need for various in-house/external training programmes and standardised processes to raise portfolio performance.

In contrast, for portfolio with high partner \& high functional diversity, there is a higher need for combining in-house alliance knowledge with external financial and legal experts in order to raise portfolio performance.

Table 7.7: Critical success factors for different alliance portfolio configurations

\begin{tabular}{|c|c|c|}
\hline $\begin{array}{l}\text { low partner } \mathcal{E} \text { high } \\
\text { functional diversity' }\end{array}$ & $\begin{array}{l}\text { high partner } \mathcal{E} \text { high } \\
\text { functional diversity }\end{array}$ & $\begin{array}{l}\text { low partner } \mathcal{E} \text { low } \\
\text { functional diversity }\end{array}$ \\
\hline \multicolumn{3}{|l|}{ alliance database $\mathrm{e}^{* * *}$} \\
\hline \multicolumn{3}{|l|}{ alliance handbook ${ }^{*}$} \\
\hline joint evaluation* & joint evaluation ${ }^{* *}$ & joint evaluation ${ }^{* *}$ \\
\hline \multicolumn{3}{|l|}{$\begin{array}{l}\text { standard partner selection } \\
\text { program* }\end{array}$} \\
\hline \multicolumn{3}{|l|}{ best practices ${ }^{* *}$} \\
\hline \multicolumn{3}{|l|}{ external alliance training ${ }^{* * *}$} \\
\hline \multicolumn{3}{|l|}{ in-house alliance training ${ }^{* *}$} \\
\hline \multicolumn{3}{|l|}{ intranet $^{* *}$} \\
\hline \multicolumn{3}{|l|}{ exchange of experience ${ }^{* *}$} \\
\hline \multicolumn{3}{|l|}{ mediators* } \\
\hline \multicolumn{3}{|l|}{ vice-presents ${ }^{* *}$} \\
\hline alliance metrics ${ }^{* *}$ & alliance metrics ${ }^{* * *}$ & \\
\hline cross alliance evaluations* & cross-alliance evaluations $^{* * *}$ & \\
\hline partner portal ${ }^{* *}$, & partner portal ${ }^{*}$ & \\
\hline culture program ${ }^{* *}$ & intercultural training** & \\
\hline \multirow[t]{6}{*}{ consultants ${ }^{* *}$} & consultants $^{* * *}$ & \\
\hline & financial experts ${ }^{* * *}$ & \\
\hline & legal experts** & \\
\hline & in-house knowledge* & \\
\hline & $\begin{array}{l}\text { alliance management develop- } \\
\text { ment program* }\end{array}$ & $\begin{array}{l}\text { alliance management } \\
\text { development program* }\end{array}$ \\
\hline & individual evaluations ${ }^{* * *}$ & individual evaluations ${ }^{* * *}$ \\
\hline
\end{tabular}




\subsection{Discussion and Conclusions}

The main objective of this chapter has been to investigate how firms can successfully address and manage alliance portfolio diversity. In line with the preceding chapters, we focussed on the firms' capability in managing alliance portfolios; that is, we examined which alliance mechanisms are most effective in managing alliance portfolio diversity.

To this end, we have empirically distinguished between three different types of alliance portfolios in terms of diversity in partner firms and functional activities and assessed how firms can raise the performance levels of these different portfolio types. ${ }^{29}$ Our empirical analysis has generated two crucial insights.

Firstly, our analysis strongly indicates the importance of selecting alliance mechanisms that are appropriate for the given type of alliance portfolio configuration. Especially, we find a considerable difference between alliance portfolios that are highly diverse in terms of partner firms and functional activities and alliance portfolios low in partner and functional diversity.

To illustrate, alliance portfolios that have a low degree of diversity in partner firms and functional activities only need to employ a few selected alliance mechanisms, such as alliance management development programs, individual and joint evaluation techniques to raise portfolio performance. In our view, this makes perfect sense. Given the focus on a single functional area and a single type of alliance partner, an alliance management development program provides the basic knowledge on alliance management, while evaluation techniques allow the firm to evaluate and monitor the performance of its alliance portfolio. Our empirical results illustrate that highly diverse alliance portfolios and low diverse alliance portfolios have in common their need for evaluation techniques to raise portfolio performance.

However, beyond this type of mechanism, highly diverse alliance portfolios need more advanced alliance mechanisms to address the managerial challenges that arise with diversity in alliance portfolios.

\footnotetext{
${ }^{29}$ While on the conceptual level we proposed four types of alliance portfolio configurations, our empirical results indicated that, most typically, firms choose among three types of alliance portfolio configurations. Noteworthy, the fourth category 'high partner diversity \& low functional diversity' does exist in our sample, but it is not a typical pattern of alliance portfolio configuration: Therefore, it did not appear in our cluster analysis.
} 
This finding contributes to the theoretical advancement of the alliance capability discipline by illustrating that the effectiveness of alliance mechanisms is indeed context-dependent, i.e. dependent on the configuration of the alliance portfolio. In the previous chapter, we have provided empirical evidence on which alliance mechanisms are best suited to address challenges of large portfolios. In the current chapter, we have included diversity as an additional dimension to alliance portfolio configuration and established which alliance mechanisms are most effective in dealing with different types of alliance portfolio diversity. Hence, our study has provided empirical evidence for the different alliance mechanisms that prove to the effective given the different configurations of alliance portfolios. Therefore, when examining how to manage alliance portfolios successfully, our study emphasises the importance of investigating the implications of size and diversity in developing effective alliance capability.

Secondly, our analysis has indicated a wide gap between what companies do in order to manage their alliance portfolios versus what is effective in managing alliance portfolios. To illustrate, firms with 'low partner $\mathcal{E}$ low functional diversity' portfolios use on average fifteen alliance mechanisms to manage their alliance portfolios (That is, they use almost as many alliance mechanisms as firms with more diverse alliance portfolios). However, by examining which alliance mechanisms are significant in raising the performance of alliance portfolios with 'low partner $\mathcal{E}$ low functional diversity', we only found three alliance mechanisms that are significant (individual evaluation, joint evaluation, alliance management development programs).

This may have two possible reasons: 1) there are no specific management problems deriving from the firms' portfolio configurations, which would require specific alliance mechanisms; or 2) firms are not aware of the specific management problems that derive from their portfolio structure and generally use such a broad set of alliance mechanisms that all differences that must be expected in this regard are simply whitewashed. Based on our empirical results in table 7.7, we must conclude that for each type of alliance portfolio configuration, certain alliance mechanisms are most effective in raising alliance portfolio performance. Therefore, regarding the gap between what firms do versus what is effective, we suspect that many firms do not know which alliance mechanisms are most effective for their particular type 
of alliance portfolio and hence many firms tend to use as many alliance mechanisms as possible.

Therefore, in practice, our findings strongly imply that firms have to carefully select those alliance mechanisms that are most suited to raise the performance of the alliance portfolio given the particular configuration of the portfolio. Our study provides a comprehensive overview of effective alliance mechanisms, which can guide firms in developing alliance portfolio capability given their specific portfolio configuration.

Overall, our findings contribute to advancement of current alliance capability research. Traditionally, alliance literature emphasised the importance of structural characteristics of the alliance portfolio to explain alliance portfolio performance. For instance, an increase in alliance portfolio diversity has usually been associated with an increase in managerial burden and complexity, and thus resulting in decreasing performance rates. However, by adopting an alliance capability view, we have directed the attention to the firm's capability in managing a portfolio of alliances. Our study strengthens current alliance capability research by applying the notion of alliance portfolio capability to the context of diversity in alliance portfolios. 


\section{CHAPTER 8 CONCLUSIONS, IMPLICATIONS AND FURTHER RESEARCH}

\subsection{Introduction}

The overarching aim of this dissertation has been to investigate how firms can attain and manage successful alliance portfolios. Having discussed various theoretical perspectives on alliance portfolio success factors, we decided to adopt an alliance capability view to studying the heterogeneity in alliance performance rates among firms. By adopting an alliance capability view, we directed our attention to the firms' internal managerial competencies and their effects on alliance performance rates. In particular, we dedicated this dissertation to answering the following research question:

How can firms develop an alliance capability that effectively supports the management of their alliance portfolios?

By answering this question, we addressed four critical research gaps in extant alliance literature. We noted that extant alliance literature:

1. is unclear about the development of alliance capability, in particular the relationship between alliance experience, alliance mechanisms and performance remains ambiguous;

2. has only provided first indications of how alliance capability on the single alliance level is different from alliance capability on the portfolio level;

3. suffers from a critical lack of large-scale empirical research to differentiate the alliance mechanisms associated with the successful management of alliance portfolios of different sizes;

4. offers only little insight on how firms can address and manage challenges that arise with diversity in alliance portfolios.

In the course of this study, we researched each of these shortcomings. 


\subsection{Main findings and conclusions}

Firstly, we addressed the issue of alliance capability building. We noted that empirical methods to proxy and measure the organizational determinants of alliance capability and assess their relationship to alliance performance tend to be frail. Therefore, we examined the relationships and effects of accumulating alliance experience and the use of alliance mechanisms on firm level alliance performance. The main findings of our empirical analysis indicate that the accumulation of alliance experience does not raise alliance performance. Rather, the use of alliance mechanisms is a stronger predictor for alliance performance. Further, we found that while some alliance mechanisms always raise alliance performance (regardless of alliance experience level) other mechanisms only raise performance in conjunction with a particular experience level. Specifically, we found that the use of individual evaluation techniques and financial experts is sufficient to raise the alliance performance for firms with little prior alliance experience. This finding was particularly interesting as it illustrated the gap between what firms do versus what is effective. In particular, inexperienced firms employ a large number of alliance mechanisms, whereas only a few have proven to be effective.

However, for companies with a moderate level of alliance experience numerous alliance mechanisms are necessary to build alliance capability: knowledge transfer mechanisms (e.g. training programmes, intranet), standardised processes (e.g. alliance evaluation techniques, alliance database, partner portals) and alliance functions (e.g. alliance department, alliance managers, vice presidents, consultants) are vital to raise alliance performance.

For companies with a high level of alliance experience, a relatively small number of mechanisms is sufficient to manage their alliances successfully, namely, in-house knowledge and cultural programmes to disseminate alliance relevant knowledge as well as alliance metrics and cross-alliance evaluations to assess the performance of their alliances.

Secondly, we investigated how alliance capability on the single alliance level is different from alliance capability on the portfolio level. So far, the exact differentiation of alliance capability on the individual versus portfolio management level had been scantily discussed. This distinction, however, is vital 
as it allows firms to understand how the management of alliance portfolios reaches beyond the tasks of managing individual alliances.

Based on a literature discussion, we delineated that the management of alliance portfolios needs to address four particular issues, including: (a) portfolio formation (i.e. setting an alliance portfolio strategy and select partners that fit the overall goal of the alliance portfolio); (b) relational governance (i.e. facilitating partner communication and interaction); (c) portfolio coordination (i.e. prioritisation and knowledge transfer between alliances within the portfolio) and (d) portfolio evaluation (i.e. monitoring and assessing the performance of the alliance portfolio as an entirety). By addressing these four tasks, companies develop a 'portfolio perspective' to managing alliances and can hereby improve the performance of the entire alliance portfolio.

Thirdly, we examined which alliance management mechanisms are most effective given the size of a firm's alliance portfolio. Our empirical analysis suggested that with an expansion in alliance portfolio size, the need for knowledge augmentation and transfer mechanisms significantly increases, as the firm recognizes the need to develop alliance portfolio capability to manage its many alliances. Especially training programs for alliance staff are found to significantly increase alliance portfolio performance. Moreover, with an expansion in portfolio size, alliance infrastructure and staff gain importance as the firm experiences a greater need for coordination of the portfolio. However, standardised processes become less important as the portfolio grows, as a large portfolio implies a variety in alliance partners and alliance types, which cannot be managed with standardised processes but need individual attention. Our findings are line with our conceptual understanding of the managerial challenges and tasks of alliance portfolios. As we derived earlier, alliance portfolio management needs to address the issues of portfolio formation, relational governance, portfolio coordination and evaluation. Indeed, we find that alliance mechanisms that are particularly geared towards the management of alliance portfolios gain significance in raising performance levels as the alliance portfolio grows.

Lastly, we examined what managerial challenges are likely to arise with alliance portfolio diversity and which alliance mechanisms are most effective at addressing these challenges. 
So far, the issue of managing alliance portfolio diversity had been scantily discussed from an alliance capability perspective. It turned out to be decisive, in this context, how diverse a firm's alliance portfolio is. The main findings of our empirical examination indicate that firms with diverse versus non-diverse alliance portfolios are in need of different alliance mechanisms in order to raise performance rates effectively. In particular, we found that firms with a high degree of alliance portfolio diversity need standardised processes such evaluation techniques and partner portals to facilitate interaction with partners; training programs such as intercultural training to provide knowledge on how to collaborate with different partner firms and alliance functions such consultants, financial experts, legal experts that support the management of the alliance portfolio. By means of these alliance mechanisms, companies can successfully manage alliance portfolio diversity.

To summarize, our study has shown that in order to build alliance capability that effectively supports the management of their alliance portfolios, firms need to design knowledge transfer mechanisms, alliance infrastructure, and standardised processes that facilitate the formation, operation and evaluation of their alliance portfolios. With growing alliance portfolios, standardised processes must be increasingly flanked by alliance-specific measures. These alliance mechanisms and measures need to be geared specifically towards addressing the managerial challenges that arise due to a firm's level of alliance experience and alliance portfolio configuration.

Table 8.1 gives an overview of the effectiveness of alliance mechanisms in relation to a firm's level of alliance experience, alliance portfolio size and diversity.

In the category of 'knowledge augmentation and transfer mechanisms', we find that the use in-house knowledge and alliance management development programs are found effective for firms with moderate/high experience level, medium portfolio size and high diversity level. The use of in-house alliance trainings and culture trainings are effective in raising portfolio performance for firms with moderate experience levels and medium to large portfolio sizes. The degree of portfolio diversity has no effect on the effectiveness of these two mechanisms.In contrast, intercultural training is found only effective in raising the performance of highly diverse portfolio. Best practices and competency framework are only found effective for firms with medium/large sizes of alliance portfolios. 
The use of intranet is found only effective when firms have a moderate level of alliance experience. Formal exchange of experience and external alliance trainings are found not effective for any level of alliance experience and portfolio configuration.

In the category of 'infrastructure and functions', we find that the use of consultants is effective for firms with moderate experience levels, medium to large portfolio sizes and highly diverse alliance portfolios. The use of vice presidents is found effective only for firms with moderate experience levels and medium portfolio size. The use of alliance managers and rewards for alliance managers is found only effective for firms with moderate experience levels. While the use of alliance specialists are only effective for firms with medium sized portfolios, financial experts benefit firms with little experience levels and highly diverse alliance portfolios. However, legal experts are only found effective when dealing with highly diverse portfolio configurations. The use of local alliance managers, rewards for business managers, mediators, gatekeepers and alliance departments are not significantly effective in raising alliance performance.

In the category of 'standardised processes', we find that the use of crossalliance evaluations and joint evaluations benefits firms with moderate/high experience levels, medium sized and highly diverse alliance portfolios. The use of individual evaluation, however, benefits from with little/moderate experience levels, small sized but highly diverse alliance portfolios. Furthermore, the use of alliance metrics is most beneficial to firms with moderate/high experience levels, small sized but highly diverse alliance portfolios. While partner portals benefit firms with moderate experience levels and highly diverse portfolios, the use of an alliance database is only found effective for firms with moderate experience levels. Joint business planning and approval processes are found only effective for firms with medium and small sized alliance portfolios, respectively. The use of standard partner selection programs, partner programs, alliance handbooks and country specific policies are not found significantly effective in raising portfolio performance. 
Reflecting upon these results, we can conclude that the effectiveness of alliance mechanisms is indeed context dependent. Alliance mechanisms that are not found effective in table 8.1 are not necessarily useless. Rather, other alliance mechanisms are found more useful in raising alliance performance

Table 8.1: Overview of effective alliance mechanisms

\begin{tabular}{|c|c|c|c|c|}
\hline & & Experience level & Portfolio size & Diversity level \\
\hline \multirow{10}{*}{ 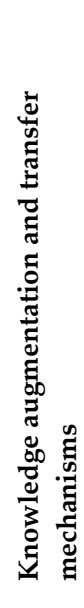 } & $\begin{array}{l}\text { Alliance management de- } \\
\text { velopment }\end{array}$ & moderate & medium & high \\
\hline & In-house knowledge & High & medium & high \\
\hline & In-house alliance training & moderate & medium, large & \\
\hline & Culture program & High & Large & \\
\hline & Intercultural training & & & high \\
\hline & Best practices & & Large & \\
\hline & Competency framework & & medium & \\
\hline & Intranet & moderate & & \\
\hline & Formal experience exchange & & & \\
\hline & External alliance training & & & \\
\hline \multirow{12}{*}{ 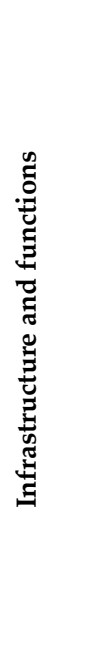 } & Consultants & moderate & medium, large & high \\
\hline & Vice president & moderate & medium & \\
\hline & Alliance managers & moderate & & \\
\hline & $\begin{array}{l}\text { Rewards for alliance } \\
\text { managers }\end{array}$ & moderate & & \\
\hline & Alliance specialist & & medium & \\
\hline & Financial experts & little & & high \\
\hline & Legal experts & & & high \\
\hline & Local alliance managers & & & \\
\hline & $\begin{array}{l}\text { Rewards for business } \\
\text { managers }\end{array}$ & & & \\
\hline & Mediators & & & \\
\hline & Gatekeeper & & & \\
\hline & Alliance department & & & \\
\hline
\end{tabular}


Table 8.1: $\quad$ Overview of effective alliance mechanisms (continued)

\begin{tabular}{|c|c|c|c|c|}
\hline & & Experience level & Portfolio size & Diversity level \\
\hline \multirow{12}{*}{ 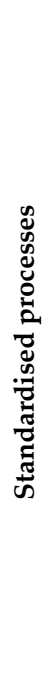 } & Cross alliance evaluation & High & medium & high \\
\hline & Joint evaluation & moderate & medium & high \\
\hline & Individual evaluation & little, moderate & small & high \\
\hline & Alliance metrics & moderate, high & small & high \\
\hline & Partner portal & moderate & & high \\
\hline & Alliance database & moderate & & \\
\hline & Joint business planning & & medium & \\
\hline & Approval processes & & small & \\
\hline & Standard partner selection & & & \\
\hline & Partner program & & & \\
\hline & Alliance handbook & & & \\
\hline & Country specific policies & & & \\
\hline
\end{tabular}

\subsection{Contributions and their theoretical and managerial implications}

First and foremost, our study has contributed to alliance capability research, both on a conceptual as well as empirical level. Our literature review on critical alliance success factors has revealed that the majority of alliance studies are still based on 'traditionally-oriented' alliance theories. While these theories rightly identify the many challenges and difficulties that can befall alliance relationships, traditional alliance studies show significant limits in their capacity to give recommendations on how firms can attain successful alliances and alliance portfolios on a continuous basis. In this respect, the alliance capability view, with its focus on the firm's internal organisation and managerial competencies, adds a crucial dimension to alliance research. By adopting an alliance capability view, we can study and explain the heterogeneity in alliance performance rates among firms and delineate how firms can improve the management and thus the performance of their alliances. For this reason, we find that the alliance capability view is an impor- 
tant emerging research theme, and it has been our aim to contribute to the development of this discipline.

In line with the alliance capability view on alliance success factors, we found that neither the size nor the structure of alliance portfolios determines the performance of the portfolio. Rather, our empirical findings strongly indicate alliance capability as the main determinant of alliance portfolio performance. On a practical level, our findings emphasise the importance of developing alliance capability for improving the performance of the alliance portfolio. That is, in contrast to the currently prevailing trial-and-error approach to managing alliances, our findings highlight the importance of adopting a dedicated, institutionalised approach to learning about alliance management and developing a repository of alliance know-how.

On a theoretical level, this finding advocates a shift away from traditional alliance studies, which solely focus on the performance implications of relational and structural characteristics of the alliance relationship.

In general, our findings advocate adopting an alliance capability view when researching alliance management and performance. By addressing shortcomings in extant alliance capability research, our study has generated three main contributions:

- Provided novel insight on the roles and interplay of alliance experience and alliance mechanisms in alliance capability building

- Contributed to the understanding of an alliance portfolio capability

- Generated empirical evidence on the effectiveness of alliance mechanisms in raising alliance performance, given a firm's

- Experience level

- Alliance portfolio configuration

Whereas the notion of alliance capability has only been introduced recently, this study deepens our understanding as it unravels how companies develop alliance capability and what role alliance mechanisms play in this respect. More specifically, this study reveals that companies need knowledge augmentation and transfer mechanisms to institutionalise prior alliance experience, they need to transform the learned lessons into standardised managerial processes and apply the lessons by means of an alliance infrastructure and staff in order to develop alliance capability. Noteworthy, our study provides a detailed description not only of which alliance mechanisms are necessary but also when these mechanisms are necessary, that is defining 
the effectiveness of alliance mechanisms given the level of a firm's alliance experience. That is, we have shown that firms can successfully learn how to manage alliances when they use alliance mechanisms appropriate to their level of alliance experience.

On a theoretical level, our findings suggest a different path to alliance capability building than laid out in extant literature. We find that firms cannot 'jump the learning curve' by using alliance training programmes as it has been suggested by other scholars. Relying on external alliance knowledge does not seem to be effective at an early stage of alliance capability building. Rather, we find that the effectiveness of integrating new knowledge depends on a firm's prior knowledge base. Hence, training programmes are less effective at this early stage as the firm has little prior knowledge in order to absorb and integrate the new knowledge effectively. Rather, the use of individual alliance evaluation techniques, as indicated by our empirical analysis, is most beneficial as it allows the firm to learn from its past mistakes; hence, this 'learning-by-doing' approach seems most appropriate given the low level of prior experience.

On a practical level, we are able to provide companies with hands-on recommendations on how to attain successful alliance portfolios. We like to draw the attention to the significant gap between what firms do to manage their alliances versus what is effective. We have witnessed this gap in two contexts: firms with different levels of alliance experience do not differ considerably with regard to the overall number of alliance mechanisms adopted. Similarly, firms with different alliance portfolios configurations do not differ considerably with regard to the overall number of alliance mechanisms adopted. It seems as if companies are not aware of the different challenges or needs that arise with different levels of alliance experience and portfolio configurations. Notably, our analysis has clearly shown that for each category of firms, different alliance mechanisms prove to be effective. One reason might be that firms try to use as many alliance mechanisms as possible due to their 'inexperience' and their aspiration to guarantee alliance success. However, building any form of organisational capability is costly. Given that firms are restricted in terms of resources and time, firms cannot afford to invest in the 'wrong' alliance mechanisms. Therefore, by indicating which alliance mechanisms are most effective in raising alliance success, our 
study gives tailor-made recommendations taking into account the companies' level of alliance experience and portfolio configuration.

\subsection{Limitations and further research}

While our findings have generated novel contributions, they raise a number of issues that require further research. First, our study inquired which alliance mechanisms are in place by companies, without verifying the extent to which these mechanisms are used. That is, we inquired the presence of alliance mechanisms and presumed that the presence of a certain mechanism implies that is it also used in the way it should be. Obviously, there is a difference between having a mechanism in place and making effective use of it. Hence, future studies can make highly relevant contributions in this area when examining the actual usage of a certain mechanism and then assessing its effectiveness on alliance performance.

Furthermore, our study did not take into account potential redundancies among our thirty-four alliance mechanisms. Our analysis assessed the effectiveness of individual alliance mechanisms, without taking into account possible interaction effects among different mechanisms. Future research can address the issue of substitutability by examining if certain alliance mechanisms can replace others, thereby limiting redundancy in knowledge transfer for example (Heimeriks, 2005).

Moreover, in our study we identified the potential managerial challenges that arise with, for instance, an expansion in alliance portfolio size by means of literature review and then tested empirically which alliance mechanisms prove to be effective given the size of the portfolio. Future research can explicitly survey companies on the challenges they experienced and ask which alliance mechanisms were found effective in addressing these issues, thereby providing more detailed recommendations on which alliance mechanisms are necessary given different tasks and phases of the alliance lifecycle.

So far, extant empirical studies, including ours, have used cross-sectional data to examine the alliance capability development process. As a result, extant literature has analysed the organisational determinants of alliance capability in a static fashion. Future research can generate highly important contributions by using longitudinal data to investigate the cyclical process of alliance capability building. Using longitudinal data may reveal potential 
interrelatedness of the various concepts used in this study. It addresses questions such as how to start building alliance capability and how alliance capability spreads through the company. Answers to these questions are not only of interest theoretically. They may also support companies in implementing their alliance capability. Longitudinal case studies into individual firms are necessary to answer these questions.

\subsection{Concluding notes}

Researching the management of alliance portfolios has proven to be a fascinating research topic. The successful management of alliance portfolios is a key challenge to companies. It allows companies to realise the full value creating potential of their alliances and thereby provides them with a competitive edge in today's economy. Adopting an alliance capability perspective to this issue has allowed us to shift away from more traditionally oriented schools of thought and examine the internal competencies and knowledge of firms in managing their portfolio of alliances. Our empirical evidence has clearly shown the importance of institutionalising alliance knowhow in the form of alliance capability for the successful management of alliance portfolios. As we have witnessed throughout the course of this study, the development of alliance capability is essential in managing alliances and thus raising the performance of firms' alliance portfolios.

This study has made important contributions to the theoretical advancement of this discipline and provides firms with hands-on recommendations on how to manage alliance portfolio successfully. Concluding, we find that the field of alliance capability is a promising and fruitful research avenue with many interesting question ahead. 



\section{REFERENCES}

\section{A}

Ahuja G. 2000. The duality of collaboration: inducements and opportunities in the formation of interfirm linkages. Strategic Management Journal 21(3): 317-343.

Aldrich HE. 1999. Organizations Evolving. Sage: London.

Anand BN, Khanna T.2000. Do Firms Learn to create value. Strategic Management Journal 21 (3) 295-315.

Anderson E, Weitz BA. 1992. The use of pledges to build and sustain commitment in distribution channels. Journal of Marketing Research 29(1): 18-34.

Anderson JC, Narus A. 1992. A Model of Distributor Firm and Manufacture Firm Working Partnerships. Journal of Marketing 54(1): 42-58.

Ansoff I. 1965. Corporate Strategy: An analytical approach to business policy of growth and expansion. McGraw-Hill: New York.

Amit R, Schoemaker PJH.1993. Strategic Assets and Organizational Rent. Strategic Management Journal 14(1):33-46.

Ariño A, de la Torre J. 1998. Learning from failure: Towards an evolutionary model of collaborative ventures. Organization Science 9(3): 306-325

Arino A, Garcia-Canal, Valdes, 1999 Longevity of strategic alliances between competitors IESE Research paper No 404. retrieved from www: http://www.iese.edu/research/pdfs/DI-0404E.pdf.

Argyris C, Schön DA. 1978. Organizational Learning: A theory of action perspective. AddisonWesley, Reading, M.A.

Argyres N, Mayer, K. 2007. Contract Design as a Firm Capability: An Integration of Learning and Transaction Cost Perspectives. Academy of Management Review 32: 1060-1077.

B

Bae J, Gargiulo M. 2004. Partner substitutability, alliance network structure, and firm profitability in the telecommunications industry. Academy of Management Journal 47(6): 843-859.

Bamford JD, Gomes Casseres B, Robinson MS. 2003. Mastering alliance strategy: A comprehensive guide to design, management, and organization. San Francisco: Jossey-Bass.

Bamford JD, Ernst D. 2002. Managing an alliance portfolio. The McKinsey Quarterly 3: 28-39.

Barkema H, Bell J, Pennings J.1996. Foreign entry, cultural barriers, and learning. Strategic Management Journal 17(2):151-166.

Barney JB. 1986. Strategic factor markets: expectations, luck, and business strategy. Management Science 32

(10):1231-1241.

Barney JD. 1991. Firm resources and sustained competitive advantage. Journal of Management 17:99-120.

Barringer BR, Harrison JS. 2000. Walking a Tightrope: Creating Value Through Interorganizational Relationships. Journal of Management 26(3):367-403.

Baum J, Calabrese T, Silverman B. 2000. Don't go it alone: alliance network composition and startups' performance in Canadian biotechnology. Strategic Management Journal 21(3): 267294. 
Baum J., Oliver C. 1991. Institutional linkages and organizational mortality. Administrative Science Quarterly 36: 187-218.

Baum J, Singh JV. 1994. Organization--environment co-evolution in Evolutionary dynamics of organizations. J Baum and J.V. Singh (eds.), 379-402. New York: Oxford University Press.

Beckman C, Haunschild P. 2002. Network learning: the effects of partners' heterogeneity of experience on corporate acquisitions. Administrative Science Quarterly 47:92-124.

Blau P. 1964. Exchange and power in social life. New York: Wiley

Blau P. 1977. Inequality and Heterogeneity: A Primitive Theory of Social Structure. Free Press, New York.

Bleeke J, Ernst D (eds). 1993. Collaborating to Compete: Using Strategic Alliances and Acquisitions in the Global Marketplace. John Wiley \& Sons: New York.

Bleeke J, Ernst D. 1991. The way to win in cross-border alliances. Harvard Business Review 69(6):127-135.

Borker M, De Man AP, Weeda P. 2004. Embedding alliance competence: alliance offices. In Van der Zee, H., Strikwerda, H (eds.) Fostering Execution, Nolan Norton Annual, De Meern, Nolan Norton \& Co, pp. 84-92.

Brown SL, Eisenhardt KM. 1997. The art of continuous change: linking complexity theory and time-paced evolution in relentlessly shifting organizations. Administrative Science Quarterly 42 (Mar.):1-34.

Burt RS. 1992. Structural Holes: The Social Structure of Competition. Cambridge, MA: Harvard University Press.

C

Child J, Faulkner D (eds.). 1998. Strategies of cooperation: Managing alliances, networks, and joint ventures. Oxford University Press.

Chung, Singh and Lee, 2000. Complementarity, status similarity and social capital as drivers of alliance formation. Strategic Management Journal 21(1): 1-22.

Coase RE. 1937. The nature of the firm. Economica 4:386-405.

Cohen WM, Levinthal DA. 1990. Absorptive capacity: A new perspective on learning and innovation. Administrative Science Quarterly 35(1): 128-152.

Cohen M, Bacdayan P.1994. Organizational routines are stored as procedural memory: evidence from a laboratory study. Organizational Science 5:554-568.

Cohen M, Burkhart R, Dosi G, Edigi M, Marengo L, Warglien M, Winter S. 1996. 'Routines and Other Recurring Action Patterns of Organisations: Contemporary Research Issues', Industrial and Corporate Change, 5: 653-698.

Coleman JS. 1990. Social Capital in Foundations of Social Theory. Cambridge, MA: Harvard University Press.

Combs JG, Ketchen DJ. 1999. Explaining Interfirm Cooperation and Performance - Toward a reconciliation of Predictions from the Resource based view and Organizational Economics. Strategic Management Journal 20(9): 867-888.

Contractor F, Lorange, P. 1988. Why should firms cooperate? The strategy and economics basis for cooperative ventures, in F.J. Contractor and P. Lorange, (Eds.): Cooperative Strategies International Business, pp.3-30, New Lexington Press, San Francisco.

Cyert RM, March JG. 1963. A Behavioural Theory of the Firm. Prentice-Hall: Englewood Cliffs, NJ.

Cowan R, David P, Foray D. 2000. The explicit economics of knowledge codification and tacitness. Industrial and Corporate Change 9: 211-253.

Crossan M, Lane H, White R. 1999. An Organizational Learning Framework: From Intuition to Institution. Academy of Management Review,24(3): 522-538. 
Dacin MT, Hitt MA, Levitas E. 1997. Selecting partners for successful international alliances: Examination of US and Korean Firms. Journal of World Business 32(1):3-16.

Das TK, Teng B. 2000. A resource based theory of strategic alliances. Journal of Management, 26: 31-61.

Das TK, Teng B. 1997. Sustaining Strategic Alliances: Options and Guidelines. Journal of General Management 22(4): 49-64.

Das TK, Teng B. 2001. Trust, Control, and Risk in Strategic Alliances: An Integrated Framework Organization Studies 22(2): 251 - 283.

Deeds DL, Hill CWL. 1996. Strategic alliances and the rate of new product development: an empirical study of entrepreneurial biotechnology firms. Journal of Business Venturing 11: 4155.

Dess G, Beard D. 1984. Dimensions of organizational task environments. Administrative Science Quarterly 29:52-73

De Man A.P. 2005. Alliance Capability A Comparison of the Alliance Strength of European and American Companies. European Management Journal 23(3): 315-323.

De Man AP, Duysters GM, Saebi T. 2010. Alliance capability as an emerging theme, in: Research in Strategic Alliances T. K. Das (eds.), City University of New York (2010).

Dosi G. 1988. Sources procedures and microeconomic effects on innovation. Journal of Economic Literature 26(3): 1120-1230.

Douma MU, Bilderbeek J, Idenburg PJ, Looise JK. 2000. Strategic Alliances: Managing the dynamics of fit. Long Range Planning 33(4): 579-598.

Doz YL. 1996. The evolution of cooperation in strategic alliances: initial conditions or learning processes? Strategic Management Journal Special Issue 17(3): 55-83.

Doz YL, Hamel G. 1998. Alliance Advantage: The Art of Creating Value through Partnering. Harvard Business School Press, Boston, Massachusetts.

Draulans J, De Man AP, Volberda HW. 1999. Alliantievaardigheid: een bron van concurrentievoordeel. Holland/Belgium Management Review 63 (January): 52-59.

Draulans J, DeMan AP, Volberda HV. 2003. Building alliance capability Management Techniques for Superior Alliance Performance. Long Range Planning 36: 151-166.

Duncen RB, Weiss A. 1979. Organizational Learning: Implications for Organizational Design. In B. M. Staw (Ed.), Research in Organizational Behavior (Vol. 1, pp. 75-123), Greenwich, CT: JAI Press.

Dussauge P, Garrette B, Mitchell W. 2000. Learning from Competing Partners: Outcomes and Durations of Scale and Link Alliances in Europe, North America and Asia. Strategic Management Journal 21(2): 99-126.

Duysters GM, De Man AP, Wildeman L. 1999. A Network Approach to Alliance Management. European Management Journal 17(2): 182-187.

Duysters GM, De Man AP. 2002. The First State of Alliance Management Study, White Paper for the Association of Strategic Alliance Professionals.

Duysters GM, De Man AP. 2007. The Second State of Alliance Management Study, White Paper for the Association of Strategic Alliance Professionals.

Dyer JH. 1996. Specialized supplier networks as a source of competitive advantage: evidence from the auto industry. Strategic Management Journal 17: 271-291.

Dyer JH, Singh H. 1998. The relational view: cooperative strategy and sources of interorganizational competitive advantage. Academy of Managing Review 23(4): 660-679.

Dyer JH, Nobeoka K. 2000. Creating and Managing a High Performance Knowledge Sharing Network: The Toyota Case. Strategic Management Journal 21(3): 345-368.

Dyer JH, Kale P, Singh H. 2001. How to make strategic alliances work. Sloan Management Review 42(4): 37-43. 


\section{E \& F}

Eisenhardt K, Martin J. 2000. Dynamic capabilities: What are they? Strategic Management Journal 21(IO.II): 1105-1121.

Eisenhardt KM, Schoonhoven CB. 1996. Resource-based view of strategic alliance formation: Strategic and social effects in entrepreneurial firms. Organisation Science 7(2): 136-150.

Eunni, R.V., Kasuganti R.R and Kos A. J (2006) Knowledge Management Processes in International Business Alliances A Review of Empirical Research, 1990-2003', International Journal of Management, 23(1).

Fiol CM, Lyles MA. 1985. Organizational learning. Academy of Management Review 10(4): 803-813.

Faulkner, D. 1995.International Strategic Alliances: Co-operating to Compete. Maidenhead: McGrawHill.

Fricke SE, Shenhar AJ. 2000. Managing multiple engineering projects in a manufacturing support environment. IEEE Transactions on Engineering Management 47(2) 258-268.

G

Gavetti, G, Levinthal D.2000. Looking Forward and Looking Backward: Cognitive and Experiential Search. Administrative Science Quarterly 45: 113-137.

George G, Zahra SA, Wheatley KK, Khan R. 2001. The effects of alliance portfolio characteristics and absorptive capacity on performance: a study of biotechnology firms. Journal of High Technology Management Research 12: 205-226.

Geringer JM, Hebert L. 1991. Measuring performance of international joint ventures. Journal of International Business Studies 22(2): 249-263.

Gimeno J. 2004. Competition within and between networks: The contingent effect of competitive embeddedness on alliance formation. Academy of Management Journal 47(6): 820-842.

Glaister KW, Buckley PJ. 1996. Strategic motives for international alliances formation. Journal of Management Studies 33: 301-332.

Goerzen A. 2007. Alliance networks and firm performance: The impact of repeated partnerships. Strategic Management Journal 28(5): 487-509.

Goerzen A, Beamish PW. 2005. The effect of alliance network diversity on multinational enterprise performance. Strategic Management Journal 26(4): 333-354.

Grant RM, Baden-Fuller C, 2004. A Knowledge Accessing Theory of Strategic Alliances. Journal of Management Studies 41: 61-84.

Grant RM, Baden-Fuller C. 2002. The knowledge-based view of strategic alliance formation: knowledge accessing versus organizational learning. In F. J. Contractor \& P. Lorange (Eds.), Cooperative strategies and alliances: 419-436. Oxford, UK: Pergamon.

Grant RM. 1996. Toward a knowledge-based theory of the firm. Strategic Management Journal, Winter Special Issue 17: 109-122.

Granovetter MS.1973 The Strength of Weak Ties The American Journal of Sociology, Vol. 78, No.6. pp.1360-1380.

Granovetter M. 1985. Economic action and social structure: the problem of embeddedness. American Journal of Sociology 91(3): 481-510.

Gulati R. 1995. Does familiarity breed trust? The implications of repeated ties for contractual choices. Academy Management Journal 35(4): 85-112.

Gulati R. 1998. Alliances and networks. Strategic Management Journal, Special Issue 19(4):293-318.

Gulati R. 1999. Network location and learning: The influence of network resources and firm capabilities on alliance formation. Strategic Management Journal 20(5): 397-420.

Gulati R, Nohria N, Zaheer A. 2000. Strategic Networks. Strategic Management Journal 21(3) Special Issue 203-215. 
Gulati R, Gargiulo M. 1999. Where do interorganizational networks come from? American Journal of Sociology 104(5): 177-231.

$\mathbf{H}$

Hagedoorn J. 1993. Understanding the rationale of strategic technology partnering. Strategic Management Journal 14(5): 371-385.

Hamel G. 1991. Competition for competence and inter-partner learning within international strategic alliances. Strategic Management Journal 12(Summer Special Issue): 83-103.

Hamel G, Doz YL, Prahalad CK. 1989. Collaborate with your competitors and win. Harvard Business Review 67(1): 133-139.

Hamel G, Heene A (eds). 1994. Competence-Based Competition. Chichester: John Wiley \& Sons.

Harbison JR, Pekar P Jr. 1998. Alliance Skills Secrets of Repeatable Success. Best Practice 11.

Harbison JR, Pekar P. 1997. Smart alliances: A practical guide to repeatable success. BoozAllen \& Hamilton. Jossey-Bass Publishers: San Francisco:

Hart SL. 1995. A natural-resource-based view of the firm. Academy of Management Review 20: 986-1014

Heimeriks KH., Duysters GM. 2007. Alliance Capability as Mediator between Experience and Alliance Performance: An Empirical Investigation into the Alliance Capability Development Process. Journal of Management Studies 44(1): 25-49.

Heimeriks KH, Duysters GM, Vanhaverbeke W. 2007. Learning mechanisms and differential performance in alliance portfolios. Strategic Organization 5(4):373-408.

Heimeriks K, Klijn E. Reuer J. 2008. Building capabilities for alliance portfolios. Long Range Planning 42 96-114.

Heimeriks, K. 2005. Developing alliance capability. Master Thesis. TU/e Eindhoven.

Henderson, R. and I. Cockburn I. 1994. Measuring competence? Exploring firm effects in pharmaceutical research. Strategic Management Journal, Winter Special Issue, 15, pp. 63-84.

Helfat, C.E. and M. A. Peteraf (2003) The Dynamic Resource-based view: Capability Lifecycles, Strategic Management Journal 24(10): 997-1010

Hennart, J.F. 1988. A transaction cost theory of equity joint ventures. Strategic Management Journal 9: 361-373.

Hennart JF, Reddy S. 1997. The choice between mergers/acquisitions and joint ventures: The case of Japanese investors in the United States. Strategic Management Journal, 18 1-12.

Hesterly WS, Liebeskind J, Zenger TR. 1990. Organizational economics: an impending revolution in organization theory? Acad. of Management Rev. 15(3) 402-420.

Hewett K, Bearden W. 2001. Dependence, trust, and relational behaviour on the part of foreign subsidiary marketing operations: Implication form managing global marketing operations. $J$ Mark 65(4):51-66.

Hitt, M.A., Dacin, A.D., Levitas, M.T., Arregle, J.L. and Borza, A. 2000. Partner selection in emerging and developed market contexts: resource-based and organizational learning perspectives. Academy of Management Journal 43(3): 449-467.

Hitt, M. A., Harrison, J. S., \& Ireland, R. D. 2001c. Mergers and acquisitions: A guide to creating value for stakeholders. New York: Oxford University Press.

Hoang HT.2001. The impact of organizational and alliance-based complexity on the development of

alliance capacity. INSEAD, White Paper.

Hoang HT., Rothaermel FT. 2005. The Effect of General and Partner-Specific Alliance Experience on Joint R\&D Project Performance. Academy of Management Journal 48(2): 332-45.

Hoffmann WH. 2005. How to manage a portfolio of alliances. Long Range Planning 38: 121-143. 
Hoffmann WH. 2007. Strategies for Managing a Portfolio of Alliances. Strategic Management Journal 28: 827-56.

Hoffmann WH, Schlosser R. 2001. Success factors of strategic alliances in small and medium sized enterprises - an empirical study. Long Range Planning 34, 357-381.

Homans G C. 1961. Social Behavior. New York, NY: Harcourt, Brace and World.

Hunt SD, Lambe CJ, Wittman CM. 2002. A Theory and Model of Business Alliance Success. Journal of Relationship Marketing 1(1) :17-36

Huxham, C. 1993: Collaborative capability: An intra-organizational perspective on collaborative advantage, Public Money \& Management July-September, 21-28.

\section{I \& J}

Inkpen, A. C. 2000. Learning through joint ventures: A framework of knowledge acquisition. Journal of Management Studies, 37(7): 1019-1043.

Inkpen AC. 1998. Learning, Knowledge Acquisition, and Strategic Alliances. European Management Journal 16(2): 223-229.

Inkpen AC, Beamish PW. 1997. Knowledge, bargaining power, and the instability of international joint ventures. Academy Management Review 22(1): 177-202.

Inkpen AC, Dinur A. 1998. Knowledge management processes and international joint ventures, Organization Science 9: 454-468.

Ireland RD, Hitt MA, Vaidyanath D. 2002. Alliance management as a source of competitive advantage. Journal of Management 28: 413-446.

Ireland RD, Hitt MA, Camp, SM, Sexton DL. 2001. Integrating entrepreneurship and strategic management thinking to create firm wealth. Academy of Management Executive 15 (1), 49-63.

Jarillo J C. 1988. On strategic networks. Strategic Management Journal 9: 31-41.

Jap S D. 1999. Pie-expansion efforts: collaboration processes in buyer-seller relationships. Journal of Marketing Research 36 (November) 461-475.

Jiang RJ, Tao QT, Santoro MD. 2010. Alliance portfolio diversity and firm performance. Strategic Management Journal 31: 1136-1144.

Joskow P. 1987. Contract duration and relationship-specific investments: empirical evidence from coal markets. American Economic Review 77(1): 168-185.

K

Kale P, Singh H. 1999. Alliance capability and success: a knowledge-based approach. Working paper Wharton School, University of Pennsylvania.

Kale P, Singh H, Perlmutter H. 2000. Learning and protection of proprietary assets in strategic alliances: Building relational capital. Strategic Management Journal 21(3): 217-237.

Kale P, Dyer J, Singh, H. 2001. Value creation and success in strategic alliances: alliancing skills and the role of alliance structure and systems. European Management Journal 19(5) 463-471.

Kale P, Dyer JH, Singh H. 2002. Alliance capability, stock market response, and long-term alliance success: the role of the alliance function. Strategic Management Journal 23(8):747-767.

Kale, P, Singh, H. 2007. Building Firm Capabilities trough Learning. Strategic Management Journal 28(10): 981-1000.

Kanter RM. 1994. Collaborative advantage. Harvard Business Review 72(4): 96-108.

Katila R, Ahuja G. 2002. Something old, something new: A longitudinal study of search behavior and new product introduction. Academy of Management Journal 45: 1189-1194.

Kenis P, Knoke D. 2002. How Organizational Field Networks Shape Interorganizational TieFormation Rates. Academy of Management Review 27:275-293. 
Kelly MJ, Schaan J, Joncas H. 2002. Managing alliance relationships Key Challenges in the early stages of collaboration. R\&D Management 32(1):11-22.

Khanna T, Gulati R, Nohria N. 1998. The dynamics of learning alliances: Competition, cooperation, and relative scope. Strategic Management Journal 19(3): 193-210.

Klein B, Murphy KM. 1988. Vertical restraints as contract enforcement mechanisms. Journal of Law and Economics, 31(2):265-297.

Klein B, Crawford R., Alchian A. 1978. Vertical integration, appropriable rents and the competitive contracting process. Journal of Law and Economics 21: 297-326.

Knoke D. 2001. Changing Organizations: Business Networks in the New Political Economy. Boulder, CO: Westview.

Kogut B. 2000: The network as knowledge: Generative rules and the emergence of structure. Strategic Management Journal, 21( Special Issue, March): 405-425.

Kogut B. 1989. The stability of joint ventures: reciprocity and competitive rivalry. Journal of Industrial Economics 38: 183-198.

Kogut B, 1988. Joint Ventures: Theoretical and Empirical Perspectives. Strategic Management Journal 9: 319-332.

Kogut B. 1991. Joint Ventures and the Option to Expand and Acquire. Management Science, 37: 19-33.

Kogut B, Singh H. 1988. The effect of national culture on the choice of entry mode. Journal. of International Business Studies 19(3): 411-432.

Kogut B, Shan W, Walker G. 1992. The make or cooperate decision in the context of an industry network. In N. Nohria and R. Eccles (eds.), Networks and Organizations. Harvard Business School Press, Boston, MA.

Kogut B, Zander U. 1992. Knowledge of the firm, combinative capabilities, and the replication of technology. Organization science 3(3): 383-397.

Kogut B, Zander U. 1996. What firms do? Coordination, identity and learning. Organization Science 7(5): 502-518.

Koka BR, Prescott JE. 2002. Strategic alliances as social capital: a multidimensional view. Strategic Management Journal 23(9):795-816.

Kor YY, Mahoney JT. 2005. How dynamics, management, and governance of resource deployments influence firm-level performance. Strategic Management Level 26(5): 489-496.

Koza MP, Lewin AY. 1998. The co-evolution of strategic alliances. Organizational Science 9(3): 255-264.

Kumar R, Nti KO. 1998. Differential learning and interaction in alliance dynamics: A process and outcome discrepancy model. Management Science 9(3): 356-367.

\section{L}

Lado AA, Boyd NB, Wright P. 1992. A competency-based model of sustainable competitive advantage. Journal of Management 18: 77-91.

Lambe CJ, Spekman RE, 1997. Alliances, External Technology Acquisition, and Discontinuous Technological Change. Journal of Product Innovation Management 14: 102-116.

Lambe CJ, Spekman RE, Hunt SD. 2002. Alliance Competence, Resources, and Alliance Success: Conceptualization, Measurement, and Initial Test. Journal of the Academy of Marketing Science 30(2): 141-158.

Lane HW, Beamish PW. 1990. Cross-cultural cooperative behaviour in joint ventures in LDCs. Management International Review 30(Special Issue): 87-102.

Lane PJ, Lubatkin M. 1998. Relative absorptive capacity and interorganizational learning. Strategic Management Journal 19(5): 461-477.

Lane PJ, Salk JE, Lyles MA. 2001. Absorptive capacity, learning, and performance in international joint 
ventures. Strategic Management Journal 22(12): 1139-1161.

Lavie D. 2006. The competitive advantage of interconnected firms: An extension of the resourcebased view. Academy of Management Review 31(3): 638-658.

Lavie D, Miller S. 2008. Alliance Portfolio Internationalization and Firm Performance. Organization Science 19(4): 623-646.

Lavie D. 2007. Alliance portfolios and firm performance: A study of value creation and appropriation in the U.S. software industry. Strategic Management Journal 28(12): 1187-1212.

Lazaric N. 1998. Trust and Organizational Learning During Inter-Firm Cooperation. In Trust and Economic Leaming. Eds. N. Lazaric and E. Lorenz. UK; Elgar. pp. 209-226.

Leonard- Barton D. 1992. Core capabilities and core rigidities: a paradox in managing new product development. Strategic Management Journal 13(Special Issue):111-125.

Leonard-Barton D. 1995. Wellsprings of knowledge. Harvard Business School Press. Mass: Boston.

Levinthal DA, March JG. 1993. The myopia of learning. Strategic Management Journal 14(Special Issue): 95-112.

Levitt B, March J. 1988. Organizational Learning. Annual Review of Sociology 14: 319-40.

Lichtenthaler U, Lichtenthaler E. 2004. Alliance functions: implications of the international multi-R\&D alliance perspective. Technovation 24: 541-552.

Lorenzoni G, Baden-Fuller C. 1995. Creating a strategic center to manage a web of partners. California Management Review 37(3):146-163.

Lu J, Beamish PW. 2006. Partnering strategies and performance of SMEs' international joint ventures. Journal of Business Venturing 21(4):461-486.

Luo Y. 2006. Opportunism in cooperative alliances: conditions and solutions. In Handbook of strategic alliances, Shenkar O, Reuer JJ (eds). Sage Publications: Thousand Oaks, CA; 55-79.

$\mathbf{M}$

Madhok A. 1995. Revisiting multinational firms' tolerance for joint ventures: a trust-based approach. Journal of International Business Studies 26(1): 117-137.

Madhok A, Tallmann S. 1998. Resource, transactions and rents: Managing value through interfirm collaborative relationships. Organization Science 9(3): 326-339.

Makadok R.2001. Towards a synthesis of the resource-based and dynamic-capability view of rent creation. Strategic Management Journal 22(5):387-401.

Mahoney JT, Pandian JR. 1992. The resource-based view within the conversation of strategic management. Strategic Management Journal 13(5):363-380.

Makino S, Beamish PW. 1998. Performance and survival of joint ventures with nonconventional ownership structures. J. Internat. Bus. Stud. 29 797-818.

March, J., 1991. Exploration and exploitation in organizational learning. Organization Science 2: 71-87.

Margulis M, Pekar P. 2001. The next wave of alliance formations: Forging successful partnerships with emerging and middle-market companies. Houlhan Lokey Howard \& Zukin.

Marino L, Strandholm K., Steensma HK, Weaver KM. 2002. Harnessing complexity: the moderating effect of national culture on entrepreneurial orientation and strategic alliance portfolio complexity. Entrepreneurship Theory and Practice, 26(4): 145-161.

Matsusaka JG. 2001. Corporate Diversification, Value Maximization, and Organizational Capabilities. Journal of Business 74 (3):409-431.

McCutchen W, Swamidass PM, Teng B. 2008. Alliance termination and performance in the biopharmaceutical industry. Journal of High Technology Management Research, 18(2) :191-202.

McEvily B, Zaheer A. 1999. Bridging ties: A source of firm heterogeneity in competitive capabilities. Strategic Management Journal 20(12): 1133-1158. 
Moingeon B, Edmondson A. 1996. Organizational Learning and Competitive Advantage. Sage, London.

Morgan RN, Hunt SD. 1994. The commitment-trust theory of relationship marketing. Journal of Marketing 58: 20-38.

Mohr J, Spekman R. 1994. Characteristics of partnership success: partnership attributes, communication behavior, and conflict resolution techniques. Strategic Management Journal 15(2):135-152.

Moorman C, Zaltman G., Deshpande R. 1992. Relationships between providers and users of marketing research: The dynamics of trust within and between organizations. Journal of Marketing Research 29(3): 314-329.

Mowery DC, Oxley JE, Silverman BS. 1996. Strategic Alliances and Interfirm Knowledge Transfer Strategic Management Journal 17 (Special Issue):77-91.

Muthusamy SK, White, M.A. 2005. Learning and Knowledge Transfer in Strategic Alliances: A Social Exchange View. Organization Studies 26 (3): 415-441.

Muthusamy SK, White MA, Carr A. 2007. An Empirical Examination of the Role of Social Exchanges in Alliance Performance. Journal of Managerial Issues XIX (1): 53-75.

$\mathbf{N}$

Narula R, Duysters GM. 2004. Globalisation and trends in international R\&D alliances. Journal of International Management 10:199-218.

Nault BR, Tyagi RK. 2001. Implementable mechanisms to coordinate horizontal alliances. Management Science 47(6): 787-7799.

Nelson RR. 1991. Why do firms differ and how does it matter? Strategic Management Journal 12 (Winter Special Issue): 61-74.

Nelson RR, Winter SG. 1982. An Evolutionary Theory of Economic Change. Harvard Business Press, Cambridge, MA.

Nonaka, I. 1994.A dynamic theory of organizational knowledge creation. Organization Science 5: 14-37.

Nonaka I, Takeuchi H. 1995. The Knowledge-Creating Firm: How Japanese Firms Create the Dynamics of Innovation. New York: Oxford University Press.

Nooteboom, B. 1996. Toward a Learning Based Model of Transactions. In Transaction Cost Economics and Beyond. Eds. J. Groenewegen. Boston, MA: Kluwer. pp. 327-350.

O

Oliver AL. 2001. Strategic alliances and the learning life-cycle of bio-technology firms. Organization Studies 22(3):467-489.

Olk P. 2002. Evaluating Strategic Alliance Performance. in F. J. Contractor and P. Lorange (eds) Cooperative Strategies and Alliances, pp. 119-43. Oxford: Elsevier Science.

Osborn, R. N., C. Baughn. 1990. Forms of interorganizational governance for multinational alliances. Acad. Management J. 33 503-519.Reuer and Leiblein 2000,

$\mathbf{P}$

Parkhe A. 1991. Interfirm diversity, organizational learning, and longevity in global strategic alliances. J.Internat. Bus. Stud 22(4) 579-601.

Parkhe A. 1993. Strategic alliance structuring: a game theoretic and transaction cost examination of interfirm cooperation. Academy of Management Journal 36(4): 794-829.

Parise S, Henderson JC. 2001. Knowledge resource exchange in strategic alliances. IBM System Journal 40(4): 908 - 924. 
Park SH, Russo MV. 1996. When competition eclipses cooperation: An event history analysis of joint venture failure. Management Science 42(6) 875-890.

Park SH, Ungson GR. 2001. Interfirm Rivalry and Managerial Complexity: A Conceptual Framework of Alliance Failure. Organization Science 12 (1):37-53.

Park SH, Ungson GR. 1997. The effect of national culture, organizational complementarity, and economic motivation on joint venture dissolution. Academy of Management Journal 40: 270-307.

Penrose ET. 1959. The theory of the growth of the firm. Oxford: Oxford University Press.

Peteraf M. 1993. The cornerstones of competitive advantage: a resource-based view. Strategic Management Journal 14(3):179-191.

Pfeffer J, Sutton RI. 1999. Knowing what do to is not enough: turning knowledge into action. California Management Review 42(1):83-108.

Pisano GP. 1989. Using equity participation to support exchange: evidence from the biotechnology industry. Journal of Law, and Organizations 5:109-126.

Pisano GP. 1994. Knowledge integration and the locus of learning: An empirical analysis of process development. Strategic Management Journal 15: 85-100.

Pisano GP, Russo MV, Teece DJ. 1988. Joint ventures and collaborative arrangement in the telecommunications equipment industry. In D.C. Mowery (ed.), International Collaborative Ventures in U.S. Manufacturing. Ballinger, Cambridge, MA, pp. 23-70.

Platje A, Seidel H, Wadman S. 1994. Project and portfolio planning cycle: project-based management for the multiproject challenge. International Journal of Project Management 12(2), 100106.

Porter ME. 1990. The competitive advantage of nations. Harvard Bus. Rev. 68(2) 73-91.

Pothukuchi V, Damanpour F, Choi J, Chen CC, Park SH. 2002. National and organizational culture differences and international joint venture performance. J. Internat. Bus. Stud 33(2) 243-265.

Powell WW, Koput KW, Smith-Doerr L. 1996. Interorganizational collaboration and the locus of innovation: Networks of learning in biotechnology. Administrative Science Quarterly 41(1), 116-145.

Prahalad CK, Hamel G. 1990. The core competence of the corporation. Harvard Business Review 68: 79-91.

Prahalad CK, Bettis RA. 1986. The dominant logic: A new linkage between diversity and performance. Strategic Management Journal 7(6), 485-502.

\section{Q \& $\mathbf{R}$}

Quynh, B.T. and Martens, R. 2008. Reducing the vulnerability of capabilities through interfirm knowledge transfer. Advances in Applied Business Strategy, 10: 159-172.

Ramanathan K, Seth A, Thomas H. 1997. Explaining joint ventures: Alternative theoretical perspectives. In P. W. Beamish \& J. P. Killing (Eds.), Cooperative Strategies: Vol. 1. North American Perspectives:51-85. San Francisco, CA: New Lexington Press.

Reed R, DeFillippi RJ. 1990. Causal ambiguity, barriers to imitation, and sustainable competitive advantage. Academy of Management Review 15: 88-102.

Reuer JJ, Arino A. 2007. Strategic alliance contracts: dimensions and determinants of contractual complexity. Strategic Management Journal 28(3): 313-330.

Reuer JJ, Ragozzino R. 2006. Agency hazards and alliance portfolios. Strategic Management Journal 27(1):27-43.

Rickert D. 1995. Multi Project Management in der industriellen Forschung und Entwicklung. DUV, Dt. Univ.-Verlag, Wiesbaden. 
Ring PS, Van de Ven AH. 1992. Structuring cooperative relationships between organizations. Strategic Management Journal 13: 483-49.

Ring PS, Van de Ven AH. 1994. Developmental Processes of Cooperative Interorganizational Relationships. Academy of Management Review 19 (1): 90-118.

Ritter, T., and Gemünden, H.G. 2003. Network competence: Its impact on innovation success and its antecedents. Journal of Business Research 56: 745-755.

Robins J, Wiersema M. 1995. A resource-based approach to the multi-business firm: Empirical analysis of portfolio inter-relationships and corporate financial performance. Strategic Management Journal 16(4):277-299.

Roth K, O'Donnell S. 1996. Foreign subsidiary compensation strategy: an agency theory perspective. Academy of Management Journal 39(3): 678-703.

Rothaermel FT. 2001. Incumbent's advantage through exploiting complementary assets via interfirm

cooperation. Strategic Management Journal 22: 687-699.

Rothaermel FT, Deeds DL. 2006. Alliance type, alliance experience and alliance management capability in high-technology ventures. Journal of Business Venturing 21:429- 460.

Rowley T, Behrens D, Krackhardt D. 2000. Redundant governance structures: an analysis of structural and relational embeddedness in the steel and semiconductor industries. Strategic Management Journal 21: 369-386.

Rumelt RP, Schendel D, Teece D.J. 1994. Fundamental issues in strategy. Boston: Harvard Business School Press.

Rumelt R, Schendel DE, Teece DJ. 1991. Strategic management and economics. Strategic Management Journal 12(Winter Special Issue): 5-29.

S

Sampson R.C. 2007. R\&D alliance and firm performance The Impact of technological diversity and alliance organizational on innovation. Academy of Management Journal 50(2): 364-386.

Sampson RC. 2005. Experience Effects and Collaborative Returns in R\&D Alliances. Strategic Management Journal 26(11): 987-1086.

Sanchez R. 2001. Managing Knowledge into Competence. The Five Learning Cycles of the competent organization. In Knowledge Management and Organizational Competence, Sanchez R (ed). Oxford University Press: Oxford, New York: 3-37.

Sanchez R., Heene A, Thomas H. 1996. Dynamics of competence-based competition. New York: Elsevier Science, Inc.

Santoro M, McGill J. 2005. The effect of uncertainty and asset co-specialization on governance in biotechnology alliances. Strategic Management Journal 26(13): 1261-1269.

Sarkar MB, Aulakh PS, Madhok A. 2009: Process capabilities and value generation in alliance portfolios. Organization Science 20(3): 583-600.

Sarkar MB, Echambadi R, Harrison J S. 2001. Alliance entrepreneurship and firm market performance. Strategic Management Journal, 22: 701-711.

Saxton T. 1997. The effects of partner and relationship characteristics on alliance outcomes. Academy of Management Journal 40(2): 443-461.

Schreiner M, Kale P, Corsten D. 2009. What really is alliance management capability and how does it impact alliance outcomes and success? Strategic Management Journal 30(13):1395-1419.

Shan W, Walker G, Kogut B. 1994. Interfirm cooperation and startup innovation in the biotechnology industry. Strategic Management Journal 15: 387-394.

Silverman BS, Baum JA. C. 2002. Alliance-based competitive dynamics. Academy of Management Journal, 45: 791-806.

Simonin BL. 1999. Ambiguity and the process of knowledge transfer in strategic alliances. Strategic Management Journal 20(7): 595-623. 
Simonin BL. 1997. The Importance of Collaborative Know-How An empirical Test of the Learning Organization. Academy of Management Journal 40(5): 1150-1174.

Sivadas E, Dwyer RF. 2000. An examination of organizational factors influencing new product development in internal and alliance-based processes. Journal of Marketing 64(1):31-49.

Smith JB, Barclay DW. 1997. The effects of organizational differences and trust on the effectiveness of selling partner relationships. Journal of Marketing 61(1), 3-21

Spekman RE, Isabella LA, MacAvoy TC. 1999. Alliance Competence: Maximizing the Value of Your Partnerships. New York: John Wiley.

Spekman, RE, Isabella LA. 2000. Alliance competence. John Wiley \& Sons, New York.

Spekman RE, Forbes TM, Isabella LA, MacAvoy TC. 1998. Alliance Management A View from the Past and Look to the Future. Journal of Management Studies 35(6): 747-772.

Spender JC. 1996. Organizational knowledge, learning, and memory: three concepts in search of a theory. Journal of Organizational Change 9:63-78.

Stearns TM, Hoffman AN, Heide .JB. 1987. Performance of commercial television stations as an outcome of interorganizational linkages and environmental conditions. Academy of Management Journal, 30, 71-90.

Stuart TE. 2000. Interorganizational alliances and the performance of firms: A study of growth and innovation rates in a high-technology industry. Strategic Management Journal 21(8) 791811.

\section{T}

Teece DJ, Pisano G. 1994. The dynamic capabilities of firms: an introduction. Industrial and Corporate Change 3(3): 537-556.

Teece DJ, Pisano G, Shuen A. 1997. Dynamic capabilities and strategic management. Strategic Management Journal 18(7). 509-533.

Teece DJ. 1992. Competition, cooperation, and innovation. Journal of Economic Behaviour and Organization 18:1-25.

Teece DJ. (Ed.). 1987. The competitive challenge: strategies for industrial innovation and renewal.

Cambridge/Mass.: Ballinger.

Tsai W., Ghoshal S. 1998. Social capital and value creation: The role of intrafirm networks. Academy of Management Journal 41: 464-476.

Todeva E, Knoke D. 2002. Strategische Allianzen und Sozialkapital von Unternehmen. Kölner Zeitschrift für Sociologie und Sozialpsychologie. Sonderheft 42:345-380.

\section{$\mathrm{U} \& \mathrm{~V}$}

Uzzi B. 1996. The sources and consequences of embeddedness for the economic performance of organizations: The net-work effect. American Sociological Review, 61: 674-698.

Uzzi B. 1997. Social structure and competition in interfirm networks: The paradox of embeddedness. Administrative Science Quarterly, 42: 35-67.

Van de Ven AH, Walker G. 1984. The dynamics of interorganizational coordination, Administrative Science Quarterly, 29, pp. 598-621.

Varadarajan, P.R., Cunningham, M.H. 1995. Strategic alliances: A synthesis of conceptual foundations. Journal of Academy of Marketing Science 23(4), 282-297.

W

Walker G., Kogut B, ShanW. 1997. Social capital, structural holes and the formation of an industry network. Organization Science 8: 109-125. 
Weitz B A, Bradford K D. 1999. Personal Selling and Sales Management: A Relationship Marketing Perspective. Journal of the Academy of Marketing Science, 27(2), 241-254.

Williamson O. 1991. Comparative economic organization: The analysis of discreet structural alternatives.

Administrative Science Quarterly 36: 269-296.

Williamson, O.E., 1975, Markets and Hierarchies: Analysis and Antitrust Implications, The Free Press, New York

Williamson, O.E., 1985, The Economic Institutions of Capitalism: Films, Markets, Relational Contracting, Free Press, New York.

Winter SG. 1987. Knowledge and competence as strategic assets. In The Competitive Challenge: Strategies for Industrial Innovation and Renewal, Teece D (ed). Ballinger: Cambridge, MA; 159184

\section{Y \& Z}

Yan, A., B. Gray. 1994. Bargaining power, management control, and performance in United States-Chinese joint ventures: A comparative case study. Acad. Management J. 37(6) 14781517.

Yoshino, M. Y. and U. S. Rangan. 1995. Strategic Alliances: An Entrepreneurial Approach to Globalization. Cambridge, MA: Harvard University Press.

Zaheer A, McEvily B, Perrone V. 1998. Does trust matter? Exploring the effects of interorganizational and interpersonal trust on performance. Organization Science 9(2): 141-159.

Zajac EJ, Olsen CP. 1993. From Transaction Cost to Transactional Value Analysis - Implications for the Study of Interorganizational Strategies. Journal of Management Studies 30(1): 131145.

Zander U, Kogut B. 1995. Knowledge and the speed of the transfer and imitation of organizational capabilities: an empirical text. Organization Science 6(1): 76-92.

Zollo M; Reuer JJ, Singh H. 2002. Interorganizational Routines and Performance in Strategic Alliances, Organization Science 13 (6) (Nov. - Dec., 2002): 701-713.

Zollo, M. and Winter, S. G. (2002) 'Deliberate Learning and the Evolution of Dynamic Capabilities', Organization Science 13(3): 339-51. 

Online Questionnaire sent to companies in 2006/2007

\section{Company Demographics}

1. Number of employees of parent company: $1-500,500-1000,>1000$

2.Total worldwide sales volume last year in US\$:

$<1$ mil., 1 m. -100 m., 100 m. -1 b., 1 b. -50 b., $>50$ b.

3. Primary industry your company is active in:

II Alliance Background

4. How many strategic alliances have you formed over the last five years?

0-5 6-15 16-25 26-40> 40+

5 . How many alliances are operational at this moment?

0-5 6-15 16-25 26-40 > 40

6.What is your company's overall alliance success rate (\% of alliances where the initial goals were realized) over the last five years?

0-20\% $21-40 \%$ 41-60\% $61-80 \% 81-100 \%$

7. What percentage of your company's market value (share price* number of shares) comes from alliances?

0-20\% $21-40 \%$ 41-60\% $61-80 \%$ 81-100\%

8. In five years, how much of your company's market value do you expect to result from alliances?

0-20\% $21-40 \%$ 41-60\% $61-80 \% 81-100 \%$

9. How important are alliances to realize your company strategy?

$1=$ not at all important; $5=$ very important

\section{Types of Alliances Formed}

10. Please indicate what percentage of your alliance portfolio are (adds to $100 \%$ ):

Co-marketing (0-100); Co-production alliances (0-100); Distribution alliances (0-100); Research alliances (0-100); Supplier alliances (0-100)

11.What percentage of your alliances includes more than two partners?

$0 \%, 1-20 \%, 21-40 \%, 41-60 \%, 61-80 \%, 81-100 \%$

12. What percentage of your alliances is with companies outside your own industry?

$0 \%, 1-20 \%, 21-40 \%, 41-60 \%, 61-80 \%, 81-100 \%$

13.What percentage of your alliances is with a competitor?

$0 \%, 1-20 \%, 21-40 \%, 41-60 \%, 61-80 \%, 81-100 \%$

14. What percentage of your company's alliances are equity alliances?

$0 \%, 1-20 \%, 21-40 \%, 41-60 \%, 61-80 \%, 81-100 \%$ 
15. What percentage of your company's research alliances is a success?

$0 \%, 1-20 \%, 21-40 \%, 41-60 \%, 61-80 \%, 81-100 \%$

16. How many international alliances have you formed over the last five years?

$0,1-5,6-15,16-25,26-40,40+$

17.What percentage of your company's international alliances is a success?

$0 \%, 1-20 \%, 21-40 \%, 41-60 \%, 61-80 \%, 81-100 \%$

IV. Alliance Capability Mechanisms: Tools

Please indicate which of the following tools are used in your company to support alliance management.

Answer option: YES / NO / Don't know

Alliance Tools

alliance best practices,

alliance database,

alliance handbook,

alliance management development program,

alliance metrics,

competency framework,

cross-alliance evaluation,

culture programme,

external alliance training,

in-house alliance training,

individual evaluation,

intranet,

joint business planning,

partner portal, partner programme,

standard partner selection approach

\section{Alliance Function}

alliance department,

alliance managers,

alliance specialist,

gatekeeper,

vice-president of alliances

Alliance Processes

formal experience exchange,

approval processes in place,

rewards and bonuses for alliance manager

External parties

consultants,

financial experts,

legal experts,

mediators for conflict resolution

\section{Alliance Portfolio in China}

We are interested in alliances formed with Chinese companies located in China. If your company has entered or is currently engaged in such an alliance, please answer the following three questions.

55. How many alliances with Chinese companies has your company formed over the last five years? 0, 1-5, 6-15, 16-25, 26-40, 40+

Considering your alliance portfolio in China, please indicate the types of alliances used (multiple answers possible):

Co-marketing, sales, co-promotion or business development alliances;

Co-production alliances (agreement to jointly produce a product)

Distribution alliances

Research alliances (alliance to jointly develop new technology, know-how)

Supplier alliances (alliance with supplier involving substantial sharing of risk and rewards)

57. What percentage of your company's Chinese alliances is a success?

$0-20 \%$ 21-40\% $41-60 \%$ 61-80\% $81-100 \%$ 
IX. Number of Alliance Professionals

58. How many fulltime alliance professionals do you estimate your company has?

59. How many people do you estimate are involved in alliances on a part time basis within your company (at least one day a week)? 



\section{SAMENVATTING}

Het doel van mijn proefschrift is te onderzoeken hoe bedrijven hun portfolio van strategische allianties succesvol kunnen managen. De toename in het aantal allianties, en het stijgende percentage van omzet gegenereerd uit allianties tonen aan dat deze samenwerkingsverbanden van toenemend belang zijn als overlevingsstrategie voor veel bedrijven.

Tegenwoordig zijn bedrijven niet meer afhankelijk van een enkele alliantie, maar vertrouwen ze in toenemende mate op een aantal externe partners om strategische onzekerheid te beheersen, toegang tot buitenlandse markten te verkrijgen, en om technologie en kennis te delen. Als gevolg hiervan zijn veel bedrijven nu ingebed in een hecht netwerk van alliantie relaties met concurrenten, leveranciers en klanten; vaak met een verscheidenheid aan industriële en nationale achtergronden.

Echter, het gemiddelde alliantie slagingspercentage van vijftig procent getuigt van de moeilijkheid om consequent successvolle allianties aan te gaan. Uit onderzoek blijkt dat bedrijven met hoge alliantie prestatie scores, superieure management vaardigheden bezitten. Deze alliantie management vaardigheden wordt "alliantievaardigheden" genoemd, en kan gezien worden als een institutionele benadering van leren binnen het alliante management teneinde de onderneming te ondersteunen bij de vorming, de werking en de evaluatie van haar allianties. Hierbij ligt de focus vanuit het perspectief van alliantievaardigheden bij de leerprocessen en management methodes die bijdragen aan het totale vermogen van de onderneming om haar allianties te managen en daarmee de prestaties verbeteren.

Uitgaande van een alliantievaardigheden perspectief, analyseren we in dit proefschrift de volgende fundamentele onderzoeksvraag: Hoe kunnen bedrijven alliantievaardigheden ontwikkelen die effectief bijdragen aan het management van hun alliantie portfolio's? Bij het beantwoorden van deze vraag heb ik een aantal kritische lacunes in de huidige literatuur over alliantievaardigheden geadresseerd. Ten eerste heb ik het conceptuele probleem geanalyseerd vanuit een theoretisch perspectief en vanuit een eigen aanpak bij het definiëren van alliantievaardigheden. Ten tweede heb ik empirisch onderzocht hoe bedrijven alliantievaardigheden kunnen opbouwen. De 
literatuur dicht een groot belang toe aan het verkrijgen van alliantie ervaring door middel van het aangaan van tal van allianties en het gebruik van organisatorische mechanismen om de ervaring te vertalen in toegankelijke lessen over alliantie management. Hiermee kunnen alliantievaardigheden worden ontwikkeld. Aangezien eerdere empirische methodes de complexiteit met betrekking tot de ontwikkeling van alliantievaardigheden niet voldoende analyseren, was het van cruciaal belang om de relatie tussen alliantie ervaring, mechanismen en de prestatie empirisch te beoordelen. Ten derde heb ik mij gericht op de ontwikkeling van alliantievaardigheden ten behoeve van alliantie portfolio management.

Hiervoor heb ik onderzoek gedaan naar de specifieke bestuurlijke uitdagingen die zich voordoen op het portfolio niveau van alliantie management. Ik heb empirisch onderzocht welke alliantie mechanismen het meest effectief zijn bij het ondersteunen van het management van grote alliantie portfolio's. Tot slot heb ik gekeken naar de verscheidene bestuurlijke uitdagingen die voorkomen bij verschillende types van alliantie portfolio diversiteit, en heb ik empirisch onderzocht welke alliantie mechanismen het meest effectief zijn bij het verhogen van de alliantie portfolio prestaties.

De belangrijkste bevindingen van deze studie tonen aan dat alliantievaardigheden ontwikkeld moeten worden die effectief management van alliantie portfolio's ondersteunt. Verder zouden bedrijven kennis overdracht mechanismen en een alliantie infrastructuur moeten ontwerpen, alsmede gestandaardiseerde processen die de vorming, werking en evaluatie van hun alliantie portfolio faciliteren moeten ontwikkelen. Deze alliantie mechanismen en maatregelen moeten specifiek worden afgestemd op de bestuurlijke uitdagingen die horen bij het niveau van ervaring met allianties en de samenstelling van de alliantie portfolio van het bedrijf. Bijgevolg heeft deze studie bijgedragen aan het onderzoek over alliantievaardigheden, zowel op conceptueel als empirisch niveau. Op een theoretisch niveau suggereren de bevindingen een andere manier om alliantievaardigheden te ontwikkelen dan door de huidige literatuur wordt voorgesteld. We concluderen dat het beroep op externe kennis niet effectief lijkt te zijn in een vroeg stadium van de opbouw van alliantievaardigheden. Met andere woorden, bedrijven kunnen geen "sprong maken op de leercurve", zoals is gesuggereerd door sommige onderzoekers. In het begin stadium lijkt juist de "leren door te doen" aanpak het meest geschikt. 
In de praktijk biedt dit onderzoek bedrijven bruikbare aanbevelingen om een succesvolle alliantie portfolio op te bouwen. Gezien het feit dat bedrijven beperkte middelen en tijd hebben, kunnen zij het zich niet veroorloven om te investeren in de "verkeerde" alliantie management praktijken. Door aan te geven welke praktijken bij welk ervaringsniveau en portfolio samenstelling het meest bijdragen aan succes, biedt deze studie een maatwerk oplossing. 



\section{CURRICULUM VitAE}

In September 2005, Tina Saebi joined the PhD program in 'Economics and Policy Studies of Technical Change' at the UNU-MERIT. Her doctoral project, entitled 'Successfully Managing Alliance Portfolios: An Alliance Capability View', commenced in September 2006 and was finalized in January 2011 under the supervision of Professor Geert Duysters and Professor ArdPieter de Man. She obtained a Masters degree in International Business Studies from Maastricht University in 2005. With Professor John Hagedoorn as her supervisor, she successfully completed her master thesis on 'Perils and Promises of ICT for Developing Countries'; particularly investigating the case of the software industry in India.

She holds a strong affiliation to the Wuhan University of Technology in China, where she conducted her field work in 2007, initialising a China-wide survey on alliance management of Chinese companies. Further, she has been involved in several consulting and training programmes on the subject of strategic alliance management, for example, working together with senior managers of the Central Bank of Nigeria as well as with alliance managers of local Dutch companies. 